\title{
Análise geoestatística multi-pontos
}

\author{
Joan Neylo da Cruz Rodriguez
}

TESE APRESENTADA

PARA O

Instituto DE MATEMÁtica E EstatísticA

DA

Universidade DE SÃo PAUlo

PARA

OBTENÇÃO DO TÍTULO

$\mathrm{DE}$

Doutor EM CIÊNCIAS

\author{
Programa: Estatística \\ Orientador: Prof. Dr. Heleno Bolfarine \\ Coorientador: Prof. Dr. Jorge Kazuo Yamamoto
}

Durante a pesquisa deste trabalho, o autor recebeu auxílio parcial da empresa Vicenza Mineração e Participações S.A. (Jan/2011 a Jul/2012) e o apoio do CNPq, Conselho Nacional de Desenvolvimento Científico e

Tecnológico - Brasil (Set/2012 a Mar/2013)

São Paulo, setembro de 2013 


\title{
Análise geoestatística multi-pontos
}

\author{
Esta tese contém as correções e alterações \\ sugeridas pela Comissão Julgadora durante a defesa \\ realizada por Joan Neylo da Cruz Rodriguez em 12/06/2013. \\ O original encontra-se disponível no Instituto de \\ Matemática e Estatística da Universidade de São Paulo.
}

Comissão Julgadora:

- Prof. Dr. Heleno Bolfarine (orientador)- IME-USP

- Prof. Dr. Jorge Kazuo Yamamoto (coorientador) - IGc-USP

- Prof. Dr. Miguel Angel Uribe Opazo - UNIOESTE

- Prof. Dr. Jorge Luis Bazán Guzmán - ICMC-USP

- Prof. Dr. Héctor Wladimir Gomez Geraldo - UA 
Dedico este trabalho:

À minha preciosa esposa, mulher, amiga e companheira de sempre Claudia de Oliveira Rodriguez.

Aos meus queridos pais (Wilfredo e Jhoncy) e meus queridos irmãos (Wilfredo, Solange, Joe, Javier e Norma).

À minha mãe Luz Ardeña Rodriguez Ramirez in memoriam, onde estejam não me esqueçam, pois sempre estarei presente. 


\section{Agradecimentos}

Gostaria de agradecer:

À minha esposa Claudia, pelo constante companheirismo, dedicação e amor, fundamentais à conclusão deste trabalho, e por ser a razão de todos os meus esforços.

Ao Professor Dr. Jorge Kazuo Yamamoto pela coorientação, pela amizade, confiança, incentivo e auxílio que tornaram possível a realização deste trabalho.

Um profundo agradecimento a meu orientador Professor Dr. Heleno Bolfarine. Ele me deu a grande oportunidade de estudar no Instituto de Geociências da Universidade de São Paulo, muito obrigado orientador por compreender e por dar-me a liberdade intelectual para prosseguir com meus interesses e por fornecer o incentivo e orientação ao longo da vida desse trabalho.

Aos meus pais (Wilfredo e Jhoncy) e meus irmãos (Wilfredo, Solange, Joe, Javier e Norma), desde que sai de casa aos 16 anos (19/12/1998), todos vocês que com muito esforço e sacrifício, me forneceram todas as condições de estudo, desde Lima e até chegar a São Paulo (04/03/2004), devo todo a vocês, fizeram possível este título de Doutor em Estatística, segue meu mais sincero afeto.

À Sra. Norma Chujutalli Tello por zelar meus irmãos durante minha ausência enquanto estudava.

À minha mãe, Luz Ardeña Rodriguez Ramirez in memoriam, hoje, mais do que nunca, sinto a tua falta, segue esse trabalho como presente.

Aos meus queridos tios Eladio Carrión, Aulalia Da Cruz e Ilda Rodriguez, me brindaram seu carinho e hospitalidade do seu lar durante a minha formação em Lima-Peru, meu maior agradecimento.

Agradeço a todos que participaram dessa minha trajetória no IME-USP, colegas de aulas e professores que me transmitiram seus conhecimentos. Tenho certeza que este trabalho não teria sido possível sem o apoio de todos vocês.

À Sala de Projetos, Bloco I, Sala 105 do Laboratório de Informática Geológica (LIG) do Departamento de Geologia Sedimentar e Ambiental e à todo o pessoal que o constitui. Agradeço em especial aos professores Jorge Kazuo Yamamoto e Marcelo Monteiro da Rocha. E aos colegas: Carlos Carrasco Arbieto, Sidney Schaberle Goveia, Fabrício Bau Dalmas, Santiago Diaz Lopez, Eduardo Takafuji e Antônio Tadashi Kikuda pelo companheirismo e bons momentos de trabalho diário.

Ao meus queridos amigos, que estudaram comigo na escola acadêmico profisional de 
estatística da Universidade Nacional Mayor de San Marcos ajudaram na realização inicial do meus objetivos (Emerson Vargas Ortega, Aléxis Lorenzo Lizárraga Paucarpura, Jesus Nilo Pajuelo Vega, Rogger Paúl Cárdenas González, Ervin Uceda Hernandez, Flor Ramos Zuñiga, Naky Lopez Ribeiro, Miriam Salazar Yabar, Andres Toyama, Walker Sixto Vasquez Aguirre, Sandra Gomez, Edith Delia Chávez Ramírez, Carlos Santana Flores) e a meus queridos professores que me formaram como estatístico, quero deixar os meus sinceros agradecimentos a todos eles, e em especial às professoras Rosario Zorina Bullón Cuadrado, Ana Maria Cárdenas Rojas, Violeta Alicia Nolberto Sifuentes, Grabiela Yolanda Montes Quintana, Rosa Fátima Medina Merino, Justa Caridad Huaroto Sumari, Olga Lídia Solano Dávila e Rosa Ysabel Adriazola Cruz e aos professores Antonio Bravo Quiroz, Manuel Rolando Canales Del Mar, Júlio César Ramos Ramirez e outros.

Ao meus queridos amigos da promoção 98 da "Institución Educativa Gómes Arias Dávila" líderes do terceiro milénio (Daniel Hidalgo Guevara, Júlio Cárdenas Pizarro, Erick Escalante Tolentino, Arquímedes Alvarado Marín, Juan Ronald Salazar, Yierlin Espinoza Funegra, Fidel Trujillo Gonzales, Paola Lopez Perez, Miluska Granda Minaya, Jenny Vargas, Boris Brioso Cajas, Ingrid Rengifo, Sherly Diaz, Karina Arévalo, Katty Caballero Dulce, Gino Tony Rojas Zevallos, continuaria listando todos vocês, mas é para todos vocês) e a meus queridos professores que me encaminharam uma boa formação fundamental e em especial a meus queridos professores Victor Estrada Mandujano e Antonio Gómez Loarte.

Ao meus queridos primos que me ajudaram na minha formação pessoal: Pilar, Cecilia, Elmer, Ruby, Franco, Javier, Luís, Cintya, Joice, Jimi, Gisela, Mónica, Fernando, Brayan, Kety, Pamelo, Henry, Katy, Anyela, Jordi e Alvaro.

Ao meus queridos professores que me ensinaram as primeiras lições, em especial a meus professores Violeta Tineo Rosales, Juana Consuelo Fretel Atencia e Jorge Chavez Arévalo da escola "Colegio Nacional Integrado Ramon Castilla de Tingo Maria".

Ao meus grandes amigos, meus irmãos de coração Adrian Enrrique Garay Velasquez, Pierre Cárdenas Ushinahua, David William Hidalgo Chavez, Juan Carlos Hidalgo Chávez, Shuver Roger Cárdenas Rengifo, Roy Cárdenas e Miguel Rios Vasquez.

À minha querida sogra Maria Auxiliadora de Oliveira pelo seu carinho e cuidado constante.

À minha querida Leticia Giovana por me aceitar como seu segundo pai, justo quando estava no final deste trabalho. E minha sobrinha Taise Dayane por sua gratidão.

Ao meus queridos e verdadeiros amigos por sempre Bruno Ledezma, Eudes Araujo, Gabriel Cabrera, Livia Fernanda, Carlos Carrasco, Dewar Carnero, Jannet Romero, Luiz Natividad, Daniela Rodrigues, Carmen Juli Sucapuca Goyzueta, Luz Goyzueta, Núbia Esteban Duarte, Josivon Souza, Pollyana Santos, Fernando Valvano Cerezetti, Karina Pretto, Pedro Fujita, Renata Rodrigues Matsuda, Roberval Ranches, Julio César Saldaña Pumarica, Yolanda Chirinos Reyes, Gleiciane da Silva Aragão, Betsabé Grimalda Blas Achic, Cristian Luis Bayes Rodriguez, Christian Danniel Paz Trillo, Jesus Mena Chalco, Jhon Edward Esquiagola Aranda, Jenny Niño de Guzman Aedo, Juan Carlos Raul Soto Sotelo, Ethel Janet 
Mercado Curi, Márcia Rezende de Oliveira, Raphael Monteiro, Aline Adelita Silveira, Lourdes Coral Contreras Montenegro, Victor Hugo Lachos e Alexandre Galvão Patriota, fico eternamente agradecidos por sua amizade e que Deus nos deixe continuar cultivando.

Ao meus queridos amigos Ivan Robert Enriquez Guzman, Cristian Noriega e Juan Carlos Cutipa Luque pelas companhias em longas horas de estudos e por fazermos um pedazinho do Peru na nossa república no início da nossa chegada ao Brasil.

Ao meus queridos afilhados Andrea Daniela Saldaña Chirinos, Thiago Alejandro Saldaña Chirinos, Benjamin Laurindo dos Santos, Claudia Yuriko Carrión Laos, Ricardo Cueva, Matheus Vinícius segue este trabalho como meu presente e exemplo para que continuem estudando a todo momento.

Aos geólogos e técnicos de campo que pertenceram ao projeto Verdete da empresa Vicenza Mineração e Participações S.A. que brindaram apoio técnico e geológico para colheita das informações que ajudaram a concepção para a aplicação do modelo estocástico, em especial à Ramayana Ferreira Viegas, Paulo Henrique Amorim Dias, Carlos Augusto da Silva Leite, Francisco Teixeira Vilela, Luciano Bruno da Rocha Oliveira, Daniel Gonçalves Sousa e João Hermelindo Laurenti.

À Claudia de Oliveira Rodriguez Diretora de Direitos Minerários da empresa Vicenza Mineração e Participações S.A. por ter proporcionado informações da legalidade das áreas para estudo e por suas constantes revisões de português.

Ao Roberto Viana Batista e Philip Yang, que por intermédio de sua empresa Vicenza Mineração e Participações S.A. brindou o apoio parcial para meu aprimoramento profissional e continuidade para a realização dos meus estudos entre janeiro de 2011 a julho de 2012 .

Ao Conselho Nacional de Desenvolvimento Científico e Tecnológico (CNPq) pela bolsa concedida para a finalização desse trabalho entre setembro de 2012 a março de 2013, processo 157850/2012-9. 


\section{Resumo}

RODRIGUEZ, J. N. C. Análise geoestatística multi-pontos. 2013. 96 f. Tese (Doutorado) - Instituto de Matemática e Estatística, Universidade de São Paulo, São Paulo, Brasil, 2013.

Estimativa e simulação baseados na estatística de dois pontos têm sido usadas desde a década de 1960 na análise geoestatístico. Esses métodos dependem do modelo de correlação espacial derivado da bem conhecida função semivariograma. Entretanto, a função semivariograma não pode descrever a heterogeneidade geológica encontrada em depósitos minerais e reservatórios de petróleo. Assim, ao invés de usar a estatística de dois pontos, a geoestatística multi-pontos, baseada em distribuições de probabilidade de múltiplo pontos, tem sido considerada uma alternativa confiável para descrição da heterogeneidade geológica. Nessa tese, o algoritmo multi-ponto é revisado e uma nova solução é proposta. Essa solução é muito melhor que a original, pois evita usar as probabilidades marginais quando um evento que nunca ocorre é encontrado no template. Além disso, para cada realização a zona de incerteza é ressaltada. Uma base de dados sintética foi gerada e usada como imagem de treinamento. A partir dessa base de dados completa, uma amostra com 25 pontos foi extraída. Os resultados mostram que a aproximação proposta proporciona realizações mais confiáveis com zonas de incerteza menores.

Palavras-chave: geoestatística, estatística multi-pontos, simulação estocástica, arvore de busca, imagem de treinamento, pixels, voxels, geometria aleatória. 


\section{Abstract}

RODRIGUEZ, J. N. C. Analysis of multiple-point geostatistics. 2013. 96 p. PhD thesis, Institute of Mathematics and Statistics, University of São Paulo, São Paulo, Brazil, 2013.

Estimation and simulation based on two-point statistics have been used since 1960's in geostatistical analysis. These methods depend on the spatial correlation model derived from the well known semivariogram function. However, the semivariogram function cannot describe the geological heterogeneity found in mineral deposits and oil reservoirs. Thus, instead of using two-point statistics, multiple-point geostatistics based on probability distributions of multiple-points has been considered as a reliable alternative for describing the geological heterogeneity. In this thesis, the multiple-point algorithm is revisited and a new solution is proposed. This solution is much better than the former one because it avoids using marginal probabilities when a never occurring event is found in a template. Moreover, for each realization the uncertainty zone is highlighted. A synthetic data base was generated and used as training image. From this exhaustive data set, a sample with 25 points was drawn. Results show that the proposed approach provides more reliable realizations with smaller uncertainty zones.

Keywords: geostatistics, multiple-point statistic, stochastic simulation, search tree, training image, pixels, voxels, random geometry. 


\section{Sumário}

$\begin{array}{ll}\text { Lista de Figuras } & \text { ix }\end{array}$

Lista de Tabelas $\quad$ xi

Lista de Abreviaturas $\quad$ xii

Lista de Símbolos $\quad$ xiii

Lista de Significados $\quad$ xiv

1 Introdução $\quad 1$

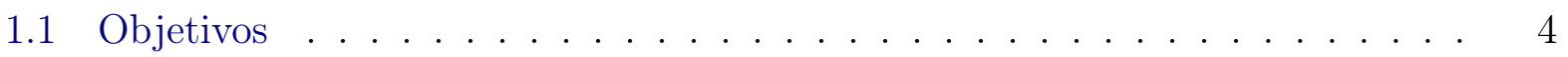

1.2 Suporte computacional . . . . . . . . . . . . . . . . . 4

2 Revisão Bibliográfica $\quad 6$

3 Conceitos $\quad 11$

3.1 Terminologia geoestatística . . . . . . . . . . . . . . . . 11

3.1 .1 Terminologia e notação . . . . . . . . . . . . . . . . . 11

3.1 .2 Variograma . . . . . . . . . . . . . . . . 12

3.1.3 Características e propriedades do variograma sob suposição de estacionariedade . . . . . . . . . . . . . . . . . . . 13

3.2 Krigagem indicadora . . . . . . . . . . . . . . . . . 15

3.2 .1 Função indicadora . . . . . . . . . . . . . . . . . 15

3.2 .2 Krigagem Indicatriz . . . . . . . . . . . . . . . . . . . . . . . 16

3.3 Terminologia geoestatística multi-pontos . . . . . . . . . . . . . . . . 18

3.4 Geoestatística multi-pontos . . . . . . . . . . . . . . . . . 19

3.5 Definição da geoestatística multi-ponto sobre a equação estendida . . . . . . 20

3.6 Digitalizando a imagem de treinamento . . . . . . . . . . . . . . . . . 22

3.7 Composição da geoestatística multi-pontos . . . . . . . . . . . . . . . . 23

4 Algoritmo $\quad 25$

4.1 Simulação da equação estendida . . . . . . . . . . . . . . . . . . . . . . 25

4.2 Algoritmo de simulação . . . . . . . . . . . . . . . . . . . . . . . . 28 
4.3 Condicionamento dos dados observados . . . . . . . . . . . . . . . . . . . . 29

4.4 Introdução da simulação multi-células . . . . . . . . . . . . . . . . . . . 30

4.5 Reprodução da função de probabilidade condicional . . . . . . . . . . . . . . 31

4.6 Código da estatística multi-pontos . . . . . . . . . . . . . . . . . . 32

5 Materiais e métodos 33

5.1 Mapeamento da árvore de probabilidades . . . . . . . . . . . . . . . 35

5.1 .1 Metodologia por Yamamoto . . . . . . . . . . . . . . . 35

5.1 .2 Metodologia por Strebelle . . . . . . . . . . . . . . . 35

5.1 .3 Nova metodologia para o estudo multi-ponto . . . . . . . . . . . . . . 36

5.2 Fluxograma multi-pontos . . . . . . . . . . . . . . . . . . . . 41

$6 \quad$ Resultados e discussão $\quad 43$

7 Conclusões $\quad 50$

7.1 Recomendações . . . . . . . . . . . . . . . . . . . . . 51

7.2 Sugestões para pesquisas futuras . . . . . . . . . . . . . . . . . 52

Referências Bibliográficas $\quad 53$

Código snesim.R por Rodriguez, J.N.C. $\quad 58$ 


\section{Lista de Figuras}

1.1 Três estruturas de dados, configurações (a), (b) e (c) utilizados para estimativa dos parâmetros do modelo de regressão linear múltipla, onde é utilizado para estimativa de valores dos blocos centrais de $100^{\prime} \times 100^{\prime}$. Gráficos segundo de Krige $(1966$, p. 22). . . . . . . . . . . . . . . .

1.2 Mapa de contornos (a) e (b) da taxa de infiltração da precipitação pluvial no solo $\left(\mathrm{mm} \mathrm{h}^{-1}\right)$ localizado numa área de $8800 \mathrm{~m}^{2}$ da Universidade de Califórnia. Gráficos segundo Vieira et al. (1981) . . . . . . . . . . . . . . . . . . . . . . .

2.1 Modelos de variogramas: exponencial (verde), gaussiano (azul) e esférico (vermelho), onde $2 \gamma(h)$ é o variograma em função da distância entre dois pontos no espaço $(h)$. E $a_{1}$ e $a_{2}$ amplitudes ou alcances, $C(0)$ a variância aleatória ou efeito pepita, $C(h)$ a variância espacial, $2 C(h)+2 C(0)$ o patamar. Gráfico modificado de Journel $(1974$, p. 679) . . . . . . . . . . . . . . . . . . .

2.2 (I) Modelo de busca sobre um arranjo hexagonal. (II) 26 distintas configurações encontradas sobre a imagem de estudo. (III) (i) Imagem em estudo, (ii) Imagem reconstruída através do algoritmo MCMC com amostrador Metropolis correspondente ao estimador de máxima verosimilhança, (iii) Imagem reconstruída através do algoritmo MCMC com amostrador Metropolis correspondente ao estimador posteriori marginal. Gráficos extraído de Tjelmeland e Besag $(1998$, p. 418, 419, 426) . . . . . . . . . . . .

3.1 Variograma e seus parâmetros: $a$ (amplitude ou alcance), $C(0)$ (variância aleatória ou efeito pepita), $C(h)$ (variância espacial), $2 C(h)+2 C(0)$ (patamar). Fonte: Yamamoto $(2001$, p. 78) . . . . . . . . . . . . . . 14

3.2 Configuração da variável $S(u)$ condicionada. . . . . . . . . . . . . . . . 20

4.1 Configuração da variável $S(u)$ condicionada a um template $\tau_{n} \operatorname{com} n=12$. . 26

4.2 Configuração da variável $S(u)$ condicionada a um template $\tau_{n}$ com $n=12$, assim como utilizado no programa. . . . . . . . . . . . . .

4.3 Configuração da variável $S(u)$ condicionada a um template $\tau_{n^{\prime}}$ num caso particular quando $n^{\prime}=10$, assim como utilizado no programa. . . . . . . . . . 
4.4 Estruturas de template de busca, configurações (I), (II), (III) e (IV), onde, captura-se informação cada vez mais abrangente.

4.5 Estruturas de template de busca em 3D, configurações (V), (VI) e (VII), onde, captura-se informação cada vez mais abrangente. . . . . . . . . . . . . . . 31

5.1 Base de dados completa constituída por 10.000 pontos. . . . . . . . . . . . . 33

5.2 Base de dados reamostrada composta por 400 pontos. . . . . . . . . . . . 34

5.3 Mapa de localização dos pontos da amostra com 25 pontos. . . . . . . . . . . 35

5.4 Tipos interpolados e mapeamento da zona de incerteza entre os tipos interpolados, conforme metodologia proposta por Yamamoto et al. (2012). . . . . 36

5.5 Fluxograma com a nova metodologia multi-pontos. . . . . . . . . . . . . 42

6.1 (a) $1^{\circ}$ realização segundo Strebelle, (b) $1^{\circ}$ realização segundo Rodriguez, (c) $2^{\circ}$ realização segundo Strebelle, (d) $2^{\circ}$ realização segundo Rodriguez, (e) $3^{\circ}$ realização segundo Strebelle, (f) $3^{\circ}$ realização segundo Rodriguez. . . . . . . .

6.2 (g) $4^{\circ}$ realização segundo Strebelle, (h) $4^{\circ}$ realização segundo Rodriguez, (i) $5^{\circ}$ realização segundo Strebelle, (j) $5^{\circ}$ realização segundo Rodriguez, (k) $6^{\circ}$ realização segundo Strebelle, (l) $6^{\circ}$ realização segundo Rodriguez. . . . . . . .

$6.3(\mathrm{~m}) 7^{\circ}$ realização segundo Strebelle, (n) $7^{\circ}$ realização segundo Rodriguez, (o) $8^{\circ}$ realização segundo Strebelle, (p) $8^{\circ}$ realização segundo Rodriguez, (q) 9 realização segundo Strebelle, (r) $9^{\circ}$ realização segundo Rodriguez. . . . . . . .

6.4 (s) $10^{\circ}$ realização segundo Strebelle, (t) $10^{\circ}$ realização segundo Rodriguez, (u) $11^{\circ}$ realização segundo Strebelle, (v) $11^{\circ}$ realização segundo Rodriguez, (w) $12^{\circ}$ realização segundo Strebelle, $(\mathrm{x}) 12^{\circ}$ realização segundo Rodriguez. . . . 


\section{Lista de Tabelas}

6.1 Frequências das litologias na imagem reamostrada de 400 pontos. . . . . . . 44

6.2 Frequências das litologias conforme as realizações multi-ponto de acordo com a metodologia de Strebelle (2000) . . . . . . . . . . . . . . . . 44

6.3 Frequências das litologias conforme as realizações multi-ponto de acordo com a nova metodologia proposta nesta tese. . . . . . . . . . . . . . . 45

6.4 Frequências das litologias conforme a realização multi-ponto de acordo com a metodologia Yamamoto et al. . . . . . . . . . . . . . 45 


\title{
Lista de Abreviaturas
}

\author{
c.c. $\quad$ Caso contrário \\ CPU Unidade central de processamento (central processing unit) \\ fp função de probabilidade (probability function) \\ fda Função de distribuição acumulada (cumulative distribution function) \\ fpc função de probabilidade condicional (conditional probability function) \\ GPS Sistema de posicionamento global (global positioning system) \\ IRF função aleatória intrínseca (intrinsic random function) \\ KI Krigagem indicadora (indicator kriging) \\ IT Imagem de treinamento (training image) \\ KS Krigagem simples (simple kriging) \\ MC Simulação de Monte Carlo (simulation of Monte Carlo) \\ MPS Metodologia geoestatística multi-pontos (multiple-point statistics) \\ RAM Memória de acesso aleatória (random access memory) \\ SNESIM Algoritmo de simulação baseada em geoestatística multi-pontos \\ por Strebelle (2002) \\ ZI Zonas de incerteza (uncertainty zones) \\ VA Variável aleatória (random variable)
}




\section{Lista de Símbolos}

$\Re^{3} \quad$ Espaço vetorial de 3 dimensões (no espaço)

$\Re^{2} \quad$ Espaço vetorial de 2 dimensões (no plano)

$E[X]$ Esperança matemática da variável aleatória $X$

$1^{\prime} 1$ pé, igual a 0,3048 metros 


\section{Lista de Significados}

Template Arranjo de células formando um modelo de busca.

Litologia

À ciência que estuda os processos de litificação, ou às

categorizações referentes a esses mesmos processos e aos tempos geológicos em que ocorreram.

Zona de Incerteza Região estabelecida por Yamamoto et al. (2012) sobre a função $p(1-p)$, onde a imagem está entre 0.20 e 0.25 . 


\section{Capítulo 1}

\section{Introdução}

Geoestatística é um ramo da estatística que une a teoria de variáveis aleatórias com a teoria de variáveis regionalizadas gerando um novo conceito de funções aleatórias. Através destas técnicas, dentre as quais se destacam a krigagem e a simulação estocástica, é possível calcular um determinado valor de interesse, onde o valor está condicionado aos dados existentes e a uma função de correlação espacial entre estes dados. Portanto, incorpora-se uma interpretação da distribuição estatística dos dados no espaço.

Assim, para entender a aparente aleatoriedade dos dados, mas com possível estruturação espacial, estabelece, desse modo, uma função de correlação espacial que representa a base da estimativa da variabilidade espacial em geoestatística, como mostrado nas equações (3.3, 3.4, 3.5 e 3.16) e da Figura 3.1.

Várias pesquisas em diversas áreas da geociências demonstram que existe uma dependência espacial nos dados amostrados. Krige (1966, p. 22) avaliou dados de mineração do ouro utilizando três estruturas, segundo um croqui de captura e disposição da localização dos dados. Esse autor fez a estimativa dos valores centrais de paneis de 100 pés quadrados, onde os parâmetros são ponderados por regressão linear múltipla através dos dados dispostos nos croquis tipo (a), (b) e (c), mostrado na Figura 1.1.

Da mesma maneira, Matheron (1971, p. 5) descreve a notação matemática das variáveis regionalizadas e sua representação das variações aleatórias e estruturais. Vieira et al. (1981, p. 1046) estudam a variabilidade espacial da taxa de infiltração da precipitação pluvial no solo $\left(\mathrm{mm} \mathrm{h}^{-1}\right)$, em uma área aproximada de $160 \mathrm{~m} \times 55 \mathrm{~m}$, contendo 1280 medidas amostradas, conseguindo com maior eficiência a estimativa de pontos não amostrados através o semivariograma experimental e a estimativa de valores pela Krigagem. Embora seja necessário conhecer a variabilidade completa da variável regionalizada, este fato deixa complexo a representação da variabilidade espacial através da mudança da distância, mas desenhados pelo mapa de contornos $^{1}$, como pode-se observar a distribuição dos valores da taxa de infiltração com os dados krigados a partir do modelo de variograma estimado, ver Figura (1.2). Trangmar et al. (1985, p. 49, 56 e 70) descrevem brevemente a teoria geoes-

\footnotetext{
${ }^{1} \mathrm{O}$ mapa de contornos é conhecido como curvas de isovalores de uma função de duas variáveis, são as curvas com equação $f(x, y)=k$, onde $k$ é uma função constante (no contra-domínio de $f$ ).
} 
tatística e sua aplicação prática na análise da variação e classificação de solos; todas estas pesquisas demonstram a necessidade de considerar na estimativa a dependência espacial, ou seja, num certo domínio, a diferença dos valores de determinada propriedade pode ser expressada como uma função da distância de separação dos pontos mensurados. Assim, quando uma propriedade varia de um local para outro com algum grau de continuidade, expressa pela correlação espacial, a geoestatística permite uma visão espacial útil ao planejamento e ao controle das informações. De forma mais abrangente e completa, a teoria de variáveis regionalizadas e as aplicações geoestatísticas são dadas por David (1977), Journel e Huijbregts (1978), Clark (1979), Isaaks e Srivastava (1989), Goovaerts (1997) e Olea (1999).

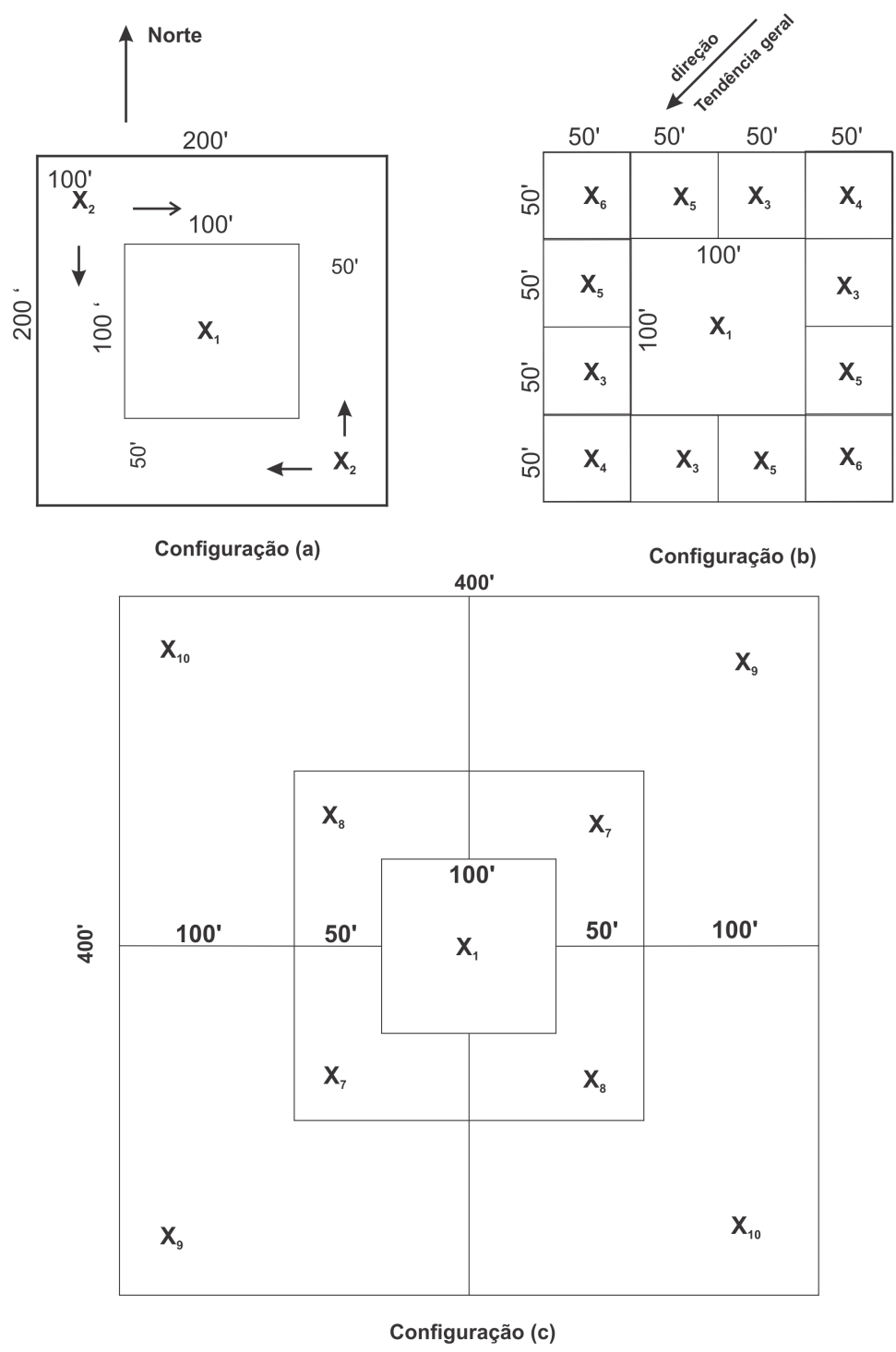

Figura 1.1: Três estruturas de dados, configurações (a), (b) e (c) utilizados para estimativa dos parâmetros do modelo de regressão linear múltipla, onde é utilizado para estimativa de valores dos blocos centrais de $100^{\prime} \times 100^{\prime}$. Gráficos segundo de Krige ( 1966, p. 22). 


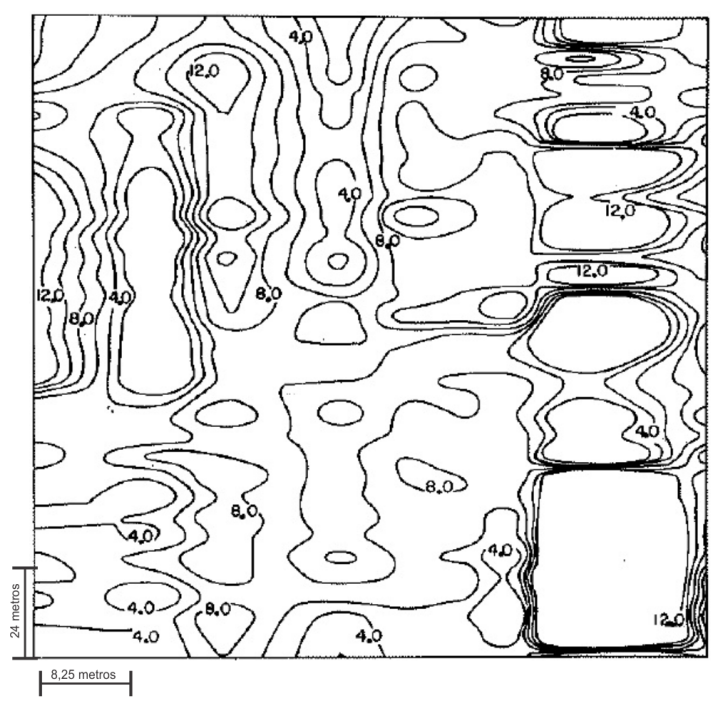

(a) Mapa de contorno construido com 1280 amostras da razão de precipitação pluvial $(\mathrm{mm} / \mathrm{hr})$. Com intervalo de contorno 2.

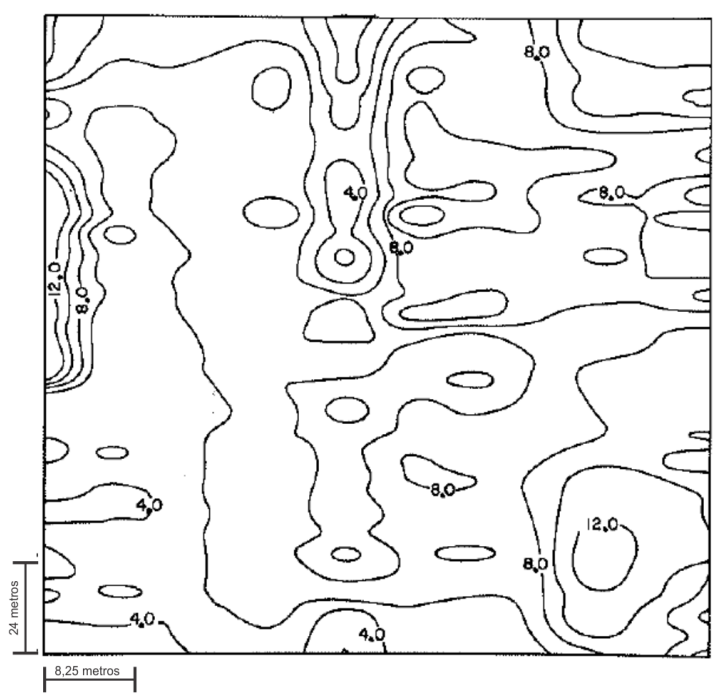

(b) Mapa de contorno construido com 1280 amostras e mais 800 amostras krigadas da razão de precipitação pluvial ( $\mathrm{mm} / \mathrm{hr})$. Com intervalo de contorno 2.

Figura 1.2: Mapa de contornos (a) e (b) da taxa de infiltração da precipitação pluvial no solo $\left(\mathrm{mm} \mathrm{h}^{-1}\right)$ localizado numa área de $8800 \mathrm{~m}^{2}$ da Universidade de Califórnia. Gráficos segundo Vieira et al. (1981).

A modelagem geoespacial permite a descrição quantitativa e qualitativa da variabilidade espacial dos atributos de interesse sobre uma determinada área ou superfície e a estimativa não tendenciosa de variância mínima de valores desses atributos em locais não amostrados. Acessar essa variabilidade, faz da geoestatística uma eficiente ferramenta de suporte a diferentes áreas tais como na medicina, biologia, agricultura, meio ambiente, dentre outras e em especial na geologia, auxiliando nos cálculos de reservas minerais na subsuperficie. Diversos são os métodos geoestatísticos utilizados no processo de geração desses mapas, entretanto, uma vez que os dados foram coletados em número razoável de pontos amostrados, é pequena a diferença em eficiência de um método para outro. Mas, para diminuir o erro de estimativa deve-se analisar e planejar o número de coletas de dados através da relação custo/benefício, pois um grande número de pontos amostrados, aumenta o custo da operação e pode inviabilizar a implantação do processo.

Para a aplicação das técnicas geoestatísticas, necessita-se, primeiramente, detalhar a área onde será implantado o estudo. Para tanto, todas as informações devem ser conhecidas e localizadas através de um processo de amostragem dentro de uma região geográfica definida. Esse processo é viabilizado pela implantação de um sistema de coordenadas locais ou geográficas, onde cada atributo ou característica de interesse coletada, sendo estas, informações quantitativas e/ou qualitativas associados a um ponto no espaço vetorial em $\Re^{2}$ ou $\Re^{3}$. Essas informações podem ser obtidas por meio de coleta in situ, mapas temáticos, imagens de satélite ou fotografias aéreas. Para referenciar essas informações, comumente utiliza-se o GPS (Sistema de Posicionamento Global), ou de forma mais simplificada através da topografia convencional, obtendo coordenadas locais. Continuando com os avanços da 
tecnologia, muitos softwares foram criados para dar suporte ao processamento das informações e ao fornecimento da variabilidade espacial dos atributos do solo e suas inter-relações com o meio, entre os softwares mais utilizados, tem-se o GeoVisual, ArcGIS, Isatis, Oasis Montaj (Geosoft), Datamine, GSLIB, Gemcom, SGeMS, PostGIS e muitos outros softwares comerciais e livres. Nem sempre os software geoestatísticos são implementados com o padrão de distribuição apresentado pelas variáveis, como os métodos geoestatísticos de interpolação, a que não necessariamente apresentam propriedades ótimas de estimativas em dados esparsos, desta forma, apresenta-se, aqui, uma alternativa a essas estimativas, em especial o da krigagem indicatriz. Desta forma, apresenta-se, aqui, uma alternativa a essas estimativas.

É muito comum encontrar trabalhos assumindo variabilidade espacial sobre a base da estatística de dois pontos em um modelo geoestatístico, ao invés de assumir variabilidade espacial com distribuições de probabilidade com mais de dois pontos. Portanto, o objetivo desta tese é estudar a metodologia da geoestatística multi-pontos para variáveis aleatórias discretas, incluindo a análise da estimativa e a análise de diagnóstico do método, e, desta forma, viabilizar a aplicabilidade dos procedimentos e algoritmos, desenvolveu-se um programa para utilizar esta metodologia de abordagem geoestatística.

\subsection{Objetivos}

A proposta deste trabalho é:

- Propor uma nova metodologia sobre as propriedades da geoestatística multi-pontos, para variáveis aleatórias qualitativas e estendida para variáveis aleatórias quantitativas discretizadas para qualquer função de probabilidade (fp);

- Implementar o algoritmo multi-pontos e procedimentos no software educacional R;

- Avaliar e estudar as propriedades dos resultados estimados desde a imagem de treinamento no template de busca selecionado (ver seção 3.6);

- Mapear a zona de incerteza da realização multi-pontos;

- Estudar a incerteza espacial do algoritmo apresentado, comparando com a tradicional técnica de krigagem indicatriz quadrática, como uma forma de validação das interpolações estimadas pela geoestatística multi-pontos.

\subsection{Suporte computacional}

A linguagem de programação $\mathbf{R}$ (versão 3.0.0 RC) constitui a plataforma computacional utilizada no desenvolvimento desta tese, que está disponível gratuitamente em http://www. r-project.org/. O $\mathbf{R}$ foi criado originalmente por Ross Ihaka e por Robert Gentleman no departamento de Estatística da Universidade de Auckland, Nova Zelândia em 1993, e desde 
meados de 1997 a equipe é composta por um corpo colaborativo de pesquisadores em vários lugares do mundo.

Esta linguagem permite a implementação de técnicas estatísticas, matemáticas e geoestatísticas com precisão e eficiência, o que tem contribuído para sua ampla utilização no campo da estatística computacional. Utilizou-se também diferentes pacotes tais como: rgl, geoR, sp, kinship2, RODBC, foreign, vegan, mvpart, Rniftilib, MASS, AnalyzeFMRI, tcltk, fastICA, shapefiles, graphics, RODBC, MASS, gstat, xlsx, gmodels, XLConnect, entre outros ambientes básicos integrados na inicialização do programa, R (2013).

$\mathrm{R}$ é um importante e poderoso veículo de análise interativa de dados que, devido à sua crescente utilização nos meios acadêmico e empresarial, não poderia deixar de implementarse a metodologia da geoestatística multi-pontos, permitindo desta forma o acesso ao open source $^{2}$ e seus respectivos conjuntos de bibliotecas $\left(\right.$ packages $\left.^{3}\right)$, onde profissionais de diferentes lugares podem usufruir deste novo recurso.

Ademais, utilizou-se os programas de ArcGis 10.1, Geosoft 7.5, GeoVisual 4.0 do Laboratório de Informática Geológica do Instituto de Geociências da Universidade de São Paulo e o sistema de processamento de texto LATEX. Para estudo de simulação utilizou-se um desktop com processador Intel(R) Core(TM) i7-2600K, CPU 3.4 GHz, 8 GB de memória RAM, sistema operacional de 64 Bits, Windows 7 Pro, patrimônio USP-IGc 044.011757.

${ }^{2}$ Código fonte aberto.

${ }^{3}$ Pacotes de programas. 


\section{Capítulo 2}

\section{Revisão Bibliográfica}

A metodologia geoestatística baseia-se na modelagem de uma função de correlação espacial, denominada variograma, a qual é utilizada posteriormente na solução de problemas de estimativa, por meio da krigagem, ou em problemas de simulação estocástica, por meio de diversos algoritmos, tais como métodos de bandas rotativas, sequenciais etc. Estes trabalhos foram iniciados por Krige (1951, p. 119 a 139) e conceituados nos diferentes trabalhos por Matheron $(1962,1963,1965,1971,1973)$ com a finalidade de fornecer estimativas precisas dos teores locais sobre os blocos de mineração, acabou desenvolvendo a formalização da metodologia geoestatística. No entanto, sua aplicação estendeu-se a muitas áreas do conhecimento, tais como a agricultura, meio ambiente, indústria petrolífera dentre outras e em especial na geologia e geofísica.

A simulação estocástica foi introduzida por Matheron (1973, p. 442, 443 e 450) ao definir as propriedades da função aleatória intrínseca (IRF, mais conhecidas como variável regionalizada) sobre o espaço de medida $\bigwedge$ e $M_{c}$, onde descreve os melhores estimadores para $k$-IRF com uma covariança generalizada e propõe o método de bandas rotativas (Turning Bands), que consiste em fazer estimativas para espaços tri-dimensionais através da simulação de linhas de dados no espaço uni-dimensional. Journel (1974, p. 675 e 679) introduz a teoria da simulação condicional e estuda a deformação da parte estrutural do variograma sobre a lei do processo gaussiano (teorema do limite central), substituindo pela função do espaço esférico e/ou exponencial, para então corrigir os efeitos de suavização e procedimentos de krigagem, permitindo a reprodução da variância espacial previsto pelo modelo de variograma, assim como mostrado na Figura (2.1), onde observa-se uma diferença na parte estrutural entre os modelos de variogramas: exponencial, gaussiano e esférico.

Srivastava (1992, p. 928 e 932) gerou diferentes realizações para a permeabilidade de um perfil de um poço de petróleo com profundidade entre $6300 \mathrm{~m}$ a $6550 \mathrm{~m}$, desde uma distribuição de probabilidade condicionada a uma localização $u$ e simulando variáreis categóricas espaciais para duas litologias, areia e xisto ${ }^{1}$, em dois poços distantes a $2000 \mathrm{~m}$. Desta

\footnotetext{
${ }^{1}$ Xisto é conhecido como Schist em inglês é o nome genérico de vários tipos de rochas metamórficas identificáveis por serem fortemente laminadas, apresenta aspecto nitidamente cristalino, e tem foliação mais ou menos nítida como resultado das fortíssimas pressões a que a rocha é sujeita.
} 
forma, o reservatório foi caracterizado com maior precisão, entre 145,4 a 204 milhões de metros cúbicos de hidrocarbonetos contidos em rocha reservatório. Goovaerts (1997, p. 369 a 436) compila várias metodologias de simulação geoestatística, sendo a base para muitas aplicações atuais geoestatísticas. Entre os algoritmos de simulação mais utilizados (Deutsch, 2002): annealing, variáveis categóricas, p-field, sequencial gaussiana e sequencial indicatriz.

Chiles e Delfiner (1999, p. 449 a 568) revisam as diferentes técnicas de simulação condicional, assim como a simulação de bandas rotativas, simulação condicional direta de variáveis continuas e simulação baseada em objetos (modelo booleano). Toda simulação estocástica fornece a capacidade de gerar múltiplas realizações equiprováveis, dando início a ideia da avaliação da incerteza espacial em dados regionalizados dado por Journel e Huijbregts (1978, p. 410) ao definir a estimativas da variância global.

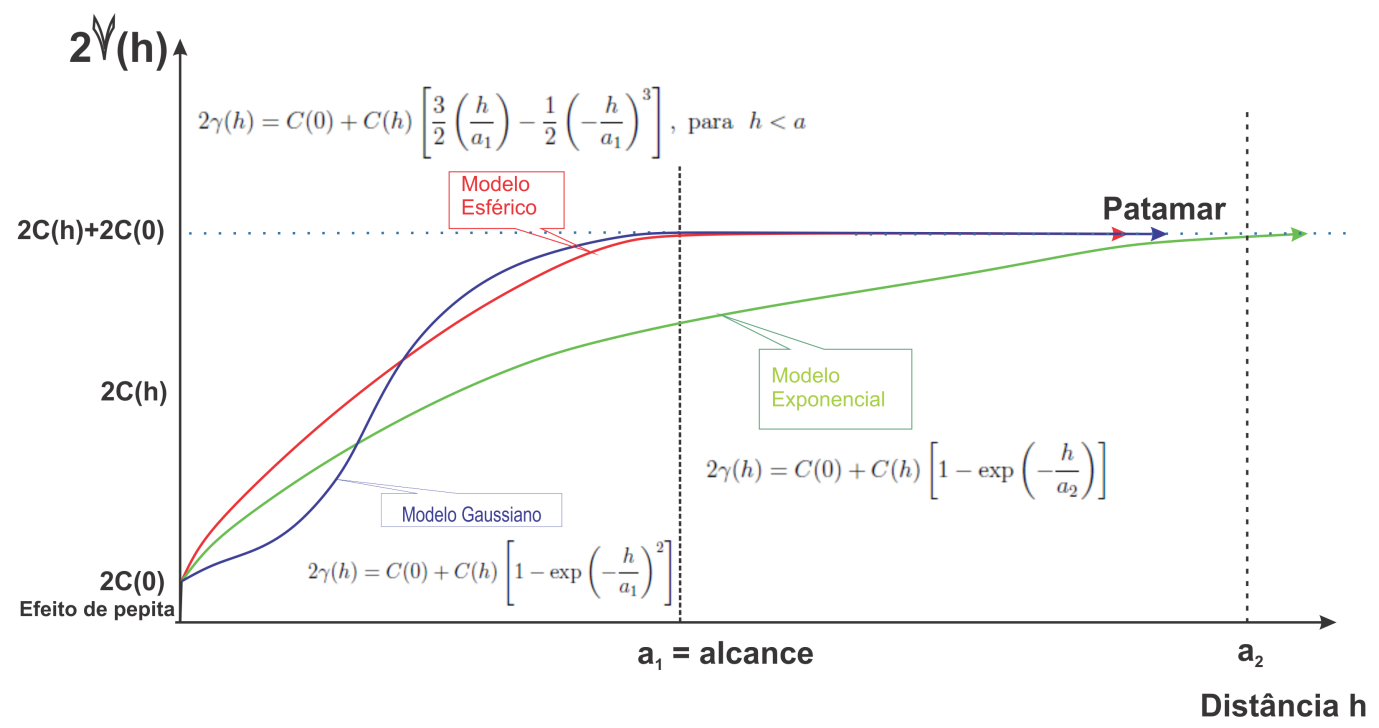

Figura 2.1: Modelos de variogramas: exponencial (verde), gaussiano (azul) e esférico (vermelho), onde $2 \gamma(h)$ é o variograma em função da distância entre dois pontos no espaço $(h)$. E $a_{1}$ e $a_{2}$ amplitudes ou alcances, $C(0)$ a variância aleatória ou efeito pepita, $C(h)$ a variância espacial, $2 C(h)+2 C(0)$ o patamar. Gráfico modificado de Journel $(1974$, p. 679).

Diferentes abordagens de simulação têm sido elaboradas na tentativa de integrar dados dinâmicos sobre estruturas de modelos de subsuperfície Descreve-se a seguir os diferentes métodos de simulação e que ainda são utilizados como, por exemplo, a metodologia baseada em gradiente estudados por Anterion et al. (1989, p. 340); assim como o método de ponto piloto estudado por Certes e De Marsily (1991, p. 288), LaVenue e Pickens (1992, p. 1544) e RamaRao et al. (1995, p. 476). O método de simulação sequencial de auto-calibração foi proposto por Gómez-Hernández et al. (1997, p. 164). Os métodos de simulação de Monte Carlo sobre campos markovianos binários em arranjos hexagonais usando um campo aleatório de Markov foram inicialmente estudados por Tjelmeland (1996, p. 2 do capítulo III) e em Tjelmeland e Besag (1998, p. 417), embora a técnica tenha uma restrição teórica definida para qualquer estatística multi ponto consistente, esta não converge de forma satisfatória para aplicações 3D, assim como mostra-se na Figura (2.2). 
Semelhantemente, a simulação de deformação gradual proposto por Roggero e Hu (1998, p. 222), onde sugerem que os campos de permeabilidade possam ser condicionados aos dados reais, como os dados dinâmicos em um sentido probabilístico, utilizando uma formulação bayesiana para a integração dados secundários.

Métodos de classificação propostos por Arpat (2005, p. 134), através do algoritmo SIM$\mathrm{PAT}^{2}$, e a metodologia proposta por Breiman et al. (1984, p. 18) no programa computacional de árvores de regressão e classificação baseados em rede neurais (CART), permitem a reprodução (similar) de eventos por interpolação dos dados encontrados.

A simulação annealing ${ }^{3}$ usada para reproduzir certas geoestatísticas multi-pontos, pois são previamente simulados a partir de uma imagem de treinamento com restrições específicas conforme uma função objetivo para cada realização multi-ponto. Além do fato de que só poucas estatísticas podem ser simultaneamente consideradas por tais restrições, a simulação annealing tem problemas de convergência relacionado à dificuldade de selecionar o melhor conjunto de parâmetros, demonstrados por Farmer (1992, p. 23) e Deutsch (1992, p. 53).

Um ramo da simulação estocástica afastou-se do variograma e da metodologia de krigagem, quando, pela primeira vez, através da elaboração do algoritmo booleano baseado em objetos (blocos, voxels), foram introduzidos por Stoyan et al. (1987, p. 67) onde apresentam resultados do método de geometria estocástica e estatísticas espaciais, incluindo as teorias básicas de processos pontuais, conjuntos aleatórios, fibras e processos de superfície, mosaicos aleatórios, estereologia e a teoria estatística de imagens sobre modelo Booleano. Haldorsen e Damsleth (1990, p. 405) discutem a importância e a necessidade de introduzir estudos relacionados a quantificação das incerteza geológica em fraturas e canais, e na avaliação de incertezas na quantificação de reservatórios de petróleo e gás natural, como uma tentativa de reproduzir características paramétricas da subsuperfície.

Inicialmente, Srivastava (1992, p. 928 e 932) com sua simulação estocástica da função de probabilidade condicional (fpc) na localização $u$, exige muito tempo de processamento nestas simulações, porque a imagem completa de treinamento tem que ser examinada novamente a cada nó não amostrado, desta maneira, ele deu o primeiro passo para o inicio de muitas pesquisas relacionados a este tema.

Posteriormente, Caers e Journel (1998, p. 323) desenvolvem a metodologia generalizada para a simulação estocástica de imagens através de redes neurais artificiais utilizando a distribuição condicional local e o amostrador de Metropolis-Hastings, onde este algoritmo produz resultados razoavelmente bons, no entanto, continua sendo de natureza iterativa, e assim demandará sempre tempo de processamento e estará propenso a problemas de convergência. Além disso, questões relacionadas com a arquitetura da rede neural tornam difícil

\footnotetext{
${ }^{2} \mathrm{O}$ objetivo principal do algoritmo SIMPAT é reproduzir padrões realísticos geológicos limitados aos dados, mas não avalia a incerteza.

${ }^{3} \mathrm{O}$ nome simulação annealing aplica-se apenas aos métodos de relaxamento estocásticos, no entanto, o uso comum do nome é usado para descrever toda a família de métodos que se baseiam no princípio de relaxamento estocástico para uma boa aproximação da função de probabilidades num espaço determinado, solucionando o problema de optimização global.
} 


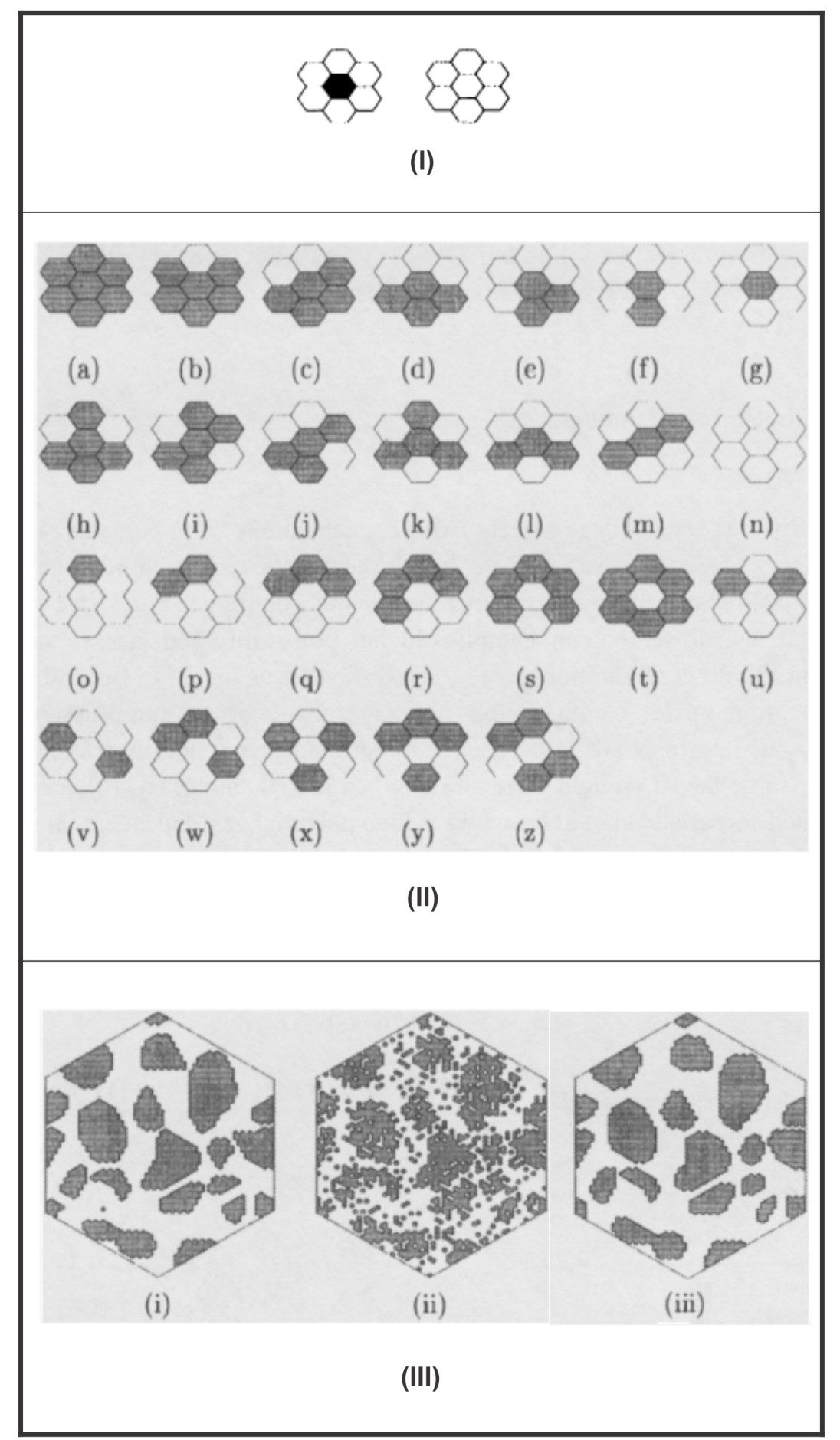

Figura 2.2: (I) Modelo de busca sobre um arranjo hexagonal.

(II) 26 distintas configurações encontradas sobre a imagem de estudo.

(III) (i) Imagem em estudo, (ii) Imagem reconstruída através do algoritmo MCMC com amostrador Metropolis correspondente ao estimador de máxima verosimilhança, (iii) Imagem reconstruída através do algoritmo MCMC com amostrador Metropolis correspondente ao estimador posteriori marginal. Gráficos extraído de Tjelmeland e Besag (1998, p. 418, 419, 426). 
o ajuste do modelo para estruturas geológicas na forma da estatística multi-pontos (MPS). Srinivasan e Caers (2000, p. 2) propuseram um procedimento baseado em rede neural da geoestatística multi-pontos (5-pontos), modelando a média da permeabilidade sobre a vizinhança do poço, filtrando as informações relacionadas à permeabilidade do campo segundo o fluxo de resposta dos dados.

Na década passada, Strebelle (2002, p. 3) propôs o uso de uma estrutura de dados de forma especial, introduzindo a terminologia da estatística multi-pontos e introduz o conceito de uma árvore de busca imbricada, onde assume as probabilidades condicionais diretamente a partir de uma imagem de treinamento, permitindo o uso da metodologia geoestatística multi-pontos e reproduzindo padrões e estruturas geológicas. As vantagens desta abordagem sobre o método Booleano baseado em objeto, incluem o processo não-iterativo de pixels e a facilidade de integração de dados de diferentes procedências e tipos, ganhando deste modo tempo de processamento e convergência.

Atualmente, os componentes básicos que caracterizam a aplicação da geoestatística multipontos continuam sendo os principais focos de pesquisa e são constantes os esforços de desenvolvimento para definir uma metodologia mais consolidada e prática. Estes componentes incluem, entre outros, a geração ou aquisição de representações espaciais numéricas a serem usadas para as imagens de treinamento. A definição ideal para a aquisição de modelos é capturar a informação condicional a partir da imagem de treinamento e o desenvolvimento de sistemas computacionais que tornam a aplicação mais prática e conveniente. Uma preocupação generalizada sobre a escolha do melhor modelo espacial leva a elaboração de diversas pesquisas sobre a dependência de modelos simulados pelas imagens de treinamento.

Os métodos das imagens de treinamento e as geoestatísticas multi-pontos se desenvolveram associados a capacidade computacional e técnicas de escaneamento, dos quais se obtinham os valores dos nós da grade. A árvore de busca, técnica de escaneamento de imagem de treinamento proposta por Strebelle (2002), permitiu uma ampla aplicação da geoestatística multi-pontos. Na abordagem através da árvore de busca, as estatísticas escaneadas são gravadas sobre uma estrutura de dados dinâmicos, onde são armazenadas informações imbricadas com o número de resultados dos eventos (probabilidades conjuntas). Devido a isto, neste trabalho, estudaremos as propriedades e as vantagens que traz esta metodologia, assim também como sua implementação mediante o software R.

Nesta última década, como anteriormente descrito, muitos trabalhos foram realizados utilizando a metodologia da estatística multi-pontos mostrando diversas aplicações, limitações e inovações da MPS, mas até o momento, não houve a construção de uma estatística que mensure as incertezas das simulações geradas pela metodologia, portanto, propõe-se a construção de estatísticas para calcular as zonas de incertezas sobre as simulações da MPS. 


\section{Capítulo 3}

\section{Conceitos}

Esta seção apresenta uma descrição da terminologia e metodologia que será utilizada para o desenvolvimento do presente trabalho.

\subsection{Terminologia geoestatística}

O conhecimento da variabilidade espacial de uma variável aleatória discreta ou contínua é a etapa fundamental para aplicar as ferramentas de estimativa por meio da Krigagem ordinária, que é o variograma. A origem das ideias foram desenvolvidas por Matheron (1971, p. 50) e sua escola de geoestatística na França, onde providencia a generalização para problemas de suavização e interpolação de superfícies espaciais desde a teoria de predição em séries temporais de Wiener-Kolmogorov, introduzindo a noção de variáveis regionalizadas, $S(u)$, como o valor $S$ de uma característica de um fenômeno geológico na localização $u$. Portanto, $S(u)$ será a denominação da variável de interesse neste trabalho.

Nas próximas seções serão fornecidas as definições de propriedades geoestatísticas e a incorporação da terminologia das geoestatísticas multi-pontos e seus paradigmas.

\subsubsection{Terminologia e notação}

Para a formação básica de um modelo geoestatístico é necessário incorporar pelo menos dois elementos:

1. Um processo estocástico com valor real $\{S(u): u \in A\}$, onde $A$ pode estar em $\Re^{d}$ no espaço $d$-dimensional e $u$ refere-se ao vetor localização. Especificamente, quando $d=1$, $A \subseteq \Re$ está no espaço unidimensional ou quando $d=2, A \subseteq \Re^{2}$ no espaço vetorial de 2 dimensões (no plano) ou quando $d=3, A \subseteq \Re^{3}$ espaço vetorial de 3 dimensões (no espaço), onde, $\Re^{3}$ será o foco do trabalho;

2. E uma distribuição multivariada para a variável aleátoria $Z=\left(Z_{1}, Z_{2}, \ldots, Z_{n}\right)^{T}$ condicionada a $S(\cdot)$, onde, $Z_{i}=S\left(u_{i}\right)$, com $i=1, \ldots, n$. Mas, é importante preservar a distinção entre o valor observável $Z_{i}$ e o não observável ou processo latente $S(u)$. 
Considerando o descrito nos itens acima, pode-se definir um modelo geoestatístico estocástico, a seguir, a definição de variograma.

\subsubsection{Variograma}

Com o variograma reconhecem-se anisotropias ${ }^{1}$, quando os variogramas se mostram diferentes para diversas direções de linhas de amostragem, como também se obtém uma ideia da variabilidade a pequenas distâncias quando o variograma experimental não for totalmente aleatório.

A função variograma $2 \gamma(h)$ é definida como a esperança matemática do quadrado da diferença entre as variáveis $Z(u+h)$ e $Z(u)$ separados por uma distância $h$, conforme a seguinte expressão:

$$
2 \gamma(h)=E\left\{[Z(u+h)-Z(u)]^{2}\right\},
$$

uma estimativa da função $2 \gamma(h)$ pode ser estimado pelo método dos momentos, a qual pode ser estimado segundo Journel (1989, p. 6-7) por:

$$
2 \widehat{\gamma(h)}=\frac{1}{n_{(h)}} \cdot \sum_{i=1}^{n_{(h)}}[Z(u+h)-Z(u)]^{2},
$$

onde, $n_{(h)}$ é o número de pares de pontos separados por uma distância $h, Z(u)$ é o valor da variável no espaço $d$-dimensional no ponto $u$, e $Z(u+h)$ é o valor da variável no espaço $d$-dimensional no ponto $u+h$. O termo variável regionalizada escolhido por Matheron (1965, p. 1) para enfatizar as feições particulares dessas variáveis, assim como na estatística pode-se definir a média (3.3), a variância (3.4) e covariância (3.5) de uma variável regionalizada, de acordo com as seguintes relações:

$$
\begin{gathered}
\mu=E[Z(u)], \\
\operatorname{Var}[Z(u)]=E\left\{[Z(u)-\mu]^{2}\right\}, \\
C(h)=E[Z(u+h) \cdot Z(u)]-\mu^{2} .
\end{gathered}
$$

A variância $\operatorname{Var}[Z(u)]$ em notação geoestatística é conhecida como $C(0)$, ou seja, a covariância para a distância nula, $h=0$.

Continuando com a equação (3.1), pode-se definir a função variograma $2 \gamma(h)$ em termos de variância $C(0)$ e de covariância $C(h)$, de acordo com o seguinte desenvolvimento:

\footnotetext{
${ }^{1}$ Anisotropia é a propriedade de dependência direcional em relação as propriedades físicas e mecânicas de um material (absorção, refracção, condutividade, resistência à tracção, etc.) onde pode ter variações graduais numa direção e rápida ou irregular em outra. De maneira oposta, a Isotropia é a propriedade que apresenta o mesmo valor e intensidade, independente de direção e sentido, Duarte (2003, p. 10 e p. 128). Estes termos são muito utilizados na mineralogia, óptica, resistência de materiais, geologia e informática.
} 


$$
2 \gamma(h)=E\left[Z^{2}(u+h)-2 Z(u+h) \cdot Z(u)-Z^{2}(u)\right]
$$

ou seja

$$
2 \gamma(h)=E\left[Z^{2}(u+h)\right]-2 E[Z(u+h) \cdot Z(u)]+E\left[Z^{2}(u)\right] .
$$

Primeiramente, desenvolvendo-se a expressão da variância através da covariância $C(h)$ quando $h=0$, tem-se:

$$
\begin{gathered}
C(0)=E[Z(u)-\mu]^{2}, \\
C(0)=E\left[Z^{2}(u)-2 Z(u) \cdot \mu+\mu^{2}\right], \\
C(0)=E\left[Z^{2}(u)\right]-2 \mu \cdot E[Z(u)]+\mu^{2},
\end{gathered}
$$

como $E[Z(u)]=\mu$ definido em 3.3, tem-se,

$$
C(0)=E\left[Z^{2}(u)\right]-2 \mu \cdot \mu+\mu^{2}=E\left[Z^{2}(u)\right]-\mu^{2}
$$

$\mathrm{ou}$

$$
E\left[Z^{2}(u)\right]=C(0)+\mu^{2} .
$$

Admitindo-se a estacionariedade, ou seja, que a média do quadrado da variável regionalizada no ponto $(u)$ é igual à variável regionalizada no ponto $(u+h)$ :

$$
E\left[Z^{2}(u)\right]=E\left[Z^{2}(u+h)\right]
$$

Substituindo-se (3.5), (3.12) e (3.13) em (3.7), a função $2 \gamma(h)$ fica:

$$
2 \gamma(h)=C(0)+\mu^{2}-2\left[C(h)+\mu^{2}\right]+C(0)+\mu^{2},
$$

então,

$$
2 \gamma(h)=2 C(0)-2 C(h)
$$

portanto, a função $\gamma(h)$ é denominada função semivariograma que é a metade da função variograma:

$$
\gamma(h)=C(0)-C(h) .
$$

\subsubsection{Características e propriedades do variograma sob suposição de estacionariedade}

Com o variograma, assim como descrito em (3.15), as variáveis regionalizadas são baseadas nos seguintes pressupostos: 
- Ergodicidade: dentro do domínio do espaço amostral gerado por todas as realizações possíveis, a esperança referente à média nas realizações será igual a média de uma única realização;

- Estacionariedade: é quando o fenômeno em estudo é dito homogêneo, sendo que dentro do espaço em que se pretende fazer estimativas, e por conveniência, assumese que os valores da área de interesse não apresentam tendência que possa afetar os resultados e assim a preocupação será apenas com a variância das diferenças entre valores das amostras;

- Hipótese íntrinseca: é quando as diferenças entre valores apresentam fraco incremento, isto é, as diferenças são localmente estacionárias determinadas apenas pela orientação espacial relativa dessas amostras, focando apenas na média e na variância das diferenças, o que significa que esses dois parâmetros dependem unicamente da orientação.

Veja na Figura (3.1) as propriedades do variograma com seus principais parâmetros que descrevem o comportamento espacial das variáveis regionalizadas.

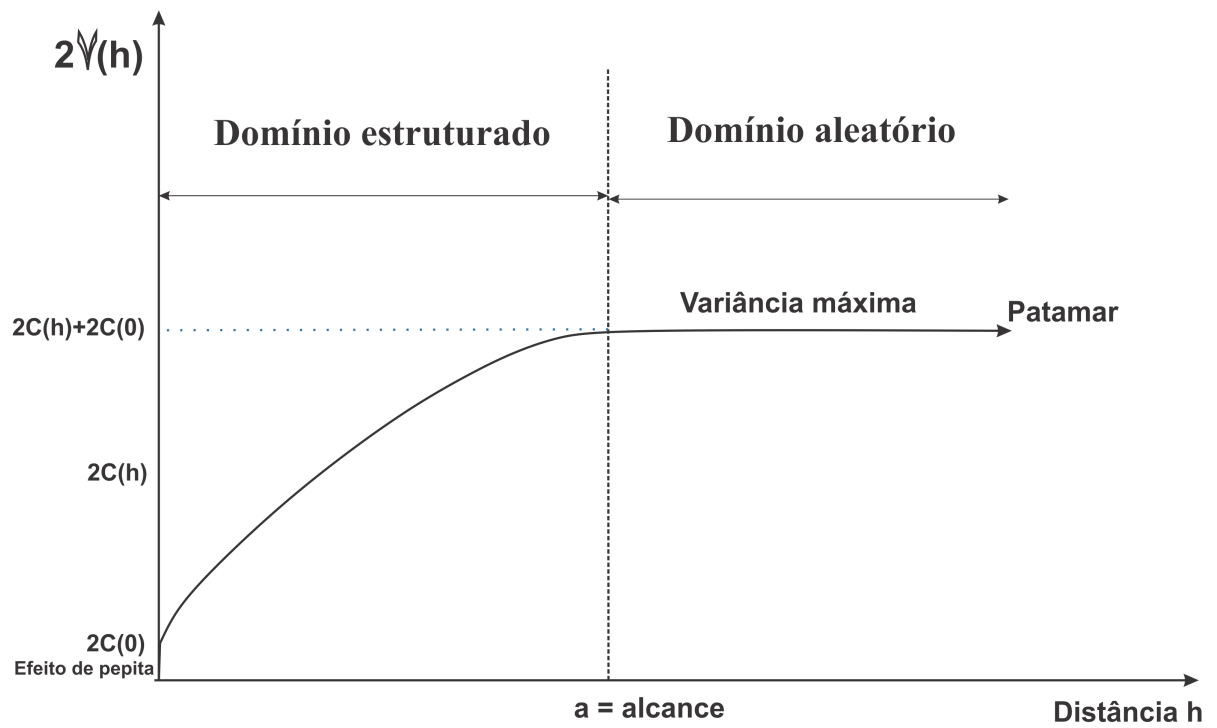

Figura 3.1: Variograma e seus parâmetros: $a$ (amplitude ou alcance), $C(0)$ (variância aleatória ou efeito pepita), $C(h)$ (variância espacial), $2 C(h)+2 C(0)$ (patamar). Fonte: Yamamoto (2001, p. 78).

Desta forma, observa-se que as características e propriedades do variograma são definidas para variáveis quantitativas contínuas e discretas e não para variáveis qualitativas, portanto, aborda-se a seguir uma alternativa à metodologia geoestatística multi-pontos para as variáveis espaciais qualitativas utilizando a krigagem indicadora.

Desta forma, observa-se que as características e propriedades do variograma são definidas para variáveis quantitativas contínuas e discretas e não para variáveis qualitativas, portanto, 
aborda-se a seguir uma alternativa para as variáveis espaciais qualitativas utilizando as metodologias da krigagem indicadora e em (3.3) a geoestatística multi-pontos.

\subsection{Krigagem indicadora}

O enfoque da tese é definir áreas com maior ou menor probabilidade onde um determinado evento ocorra através do estudo das variáveis categóricas, ao invés de estimar um determinado valor. Entretanto, na krigagem ordinária, as variâncias são condicionadas apenas pelo arranjo geométrico dos pontos e independente dos valores das amostras. Estes valores não apresentam medidas de exatidão da estimativa local, portanto, existe a necessidade de trabalhar com a metodologia krigagem indicadora (KI), usando informações do modelo de variograma.

A KI é entendida como uma técnica de análise de regressão que procura minimizar a variância estimada a partir de um modelo prévio considerando a dependência estocástica entre os dados distribuídos no espaço. Assim, a variância da KI considerando o estimador linear não-viesado (ELNV) será utilizada para definir intervalos de confiança, da mesma maneira como é utilizada para os modelos gaussianos.

O conceito inicial foi apresentado por Journel (1982, p. 795 e 1983, p. 447) como uma proposta para construir uma função de distribuição acumulada (fda) para a estimativa de distribuições espaciais. O conceito da transformação indicadora e os variogramas das indicadoras acabam simplificando a modelagem.

A seguir a descrição da KI que será utilizada ao longo deste trabalho.

\subsubsection{Função indicadora}

Seja $S(u)$ uma variável aleatória (VA) com $k$ categorias, na localização $u$, onde a função indicadora para a $k$-ésima categoria será definida como:

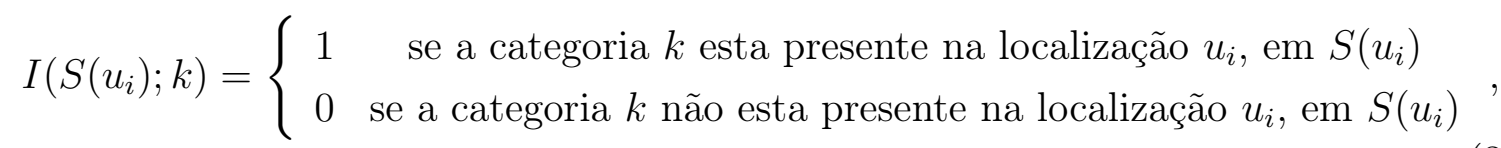

onde, $i=1, \ldots, n$.

A função indicadora $I\left(S\left(u_{i}\right) ; k\right)$ é também conhecida como variável de Bernoulli² ${ }^{2}$ nessas condições a v.a. $I\left(S\left(u_{i}\right) ; k\right)$ tem distribuição de Bernoulli, e sua função de probabilidade para cada $i$ é dada por:

$$
P\left[I\left(S\left(u_{i}\right) ; k\right)=\ell\right]=p_{k}^{\ell}\left(1-p_{k}\right)^{1-\ell}, \ell=0,1 .
$$

\footnotetext{
${ }^{2}$ A variável Bernoulli definida dentro do espaço amostral $\{0,1\}$ com localização $u_{i}$, e com suas respectivas funções de probabilidades, definidas como $P\left[I\left(S\left(u_{i}\right) ; k\right)=1\right]=p_{k}$ e $P\left[I\left(S\left(u_{i}\right) ; k\right)=0\right]=1-p_{k}$. O nome da distribuição se refere ao cientista suiço Jakob Bernoulli.
} 
Desta forma, encontra-se a esperança matemática (3.21) e a variância (3.27) da variável $I\left(S\left(u_{i}\right) ; k\right)$, dada por:

$$
\begin{aligned}
E\left[I\left(S\left(u_{i}\right) ; k\right)\right]= & \left(I\left(S\left(u_{i}\right) ; k\right)=1\right) \times P\left[I\left(S\left(u_{i}\right) ; k\right)=1\right]+ \\
& \left(I\left(S\left(u_{i}\right) ; k\right)=0\right) \times P\left[I\left(S\left(u_{i}\right) ; k\right)=0\right] \\
= & 1 \times p_{k}+0 \times\left(1-p_{k}\right) \\
E\left[I\left(S\left(u_{i}\right) ; k\right)\right]= & p_{k},
\end{aligned}
$$

onde, o $p_{k}$ da função indicadora pode ser obtida pela razão $p_{k}=f_{k} / N$, onde $f_{k}$ é a frequência da $k$-ésima categoria e $N=\sum_{k=1}^{K} f_{k}$ é o número total dentro do domínio.

E a variância da função indicadora é

$$
\begin{aligned}
V\left[I\left(S\left(u_{i}\right) ; k\right)\right]= & E\left[I\left(S\left(u_{i}\right) ; k\right)-p_{k}\right]^{2} \\
= & E\left[I^{2}\left(S\left(u_{i}\right) ; k\right)-2 p_{k} I\left(S\left(u_{i}\right) ; k\right)+p_{k}^{2}\right] \\
= & E\left[I\left(S\left(u_{i}\right) ; k\right)\right]-2 p_{k} E\left[I\left(S\left(u_{i}\right) ; k\right)\right]+p_{k}^{2}, \operatorname{como} I\left(S\left(u_{i}\right) ; k\right) \text { é }(3.24) \\
& \text { definida como uma v.a. Bernoulli, então } I^{2}\left(S\left(u_{i}\right) ; k\right)=I\left(S\left(u_{i}\right) ; k\right) . \\
= & p_{k}-2 p_{k} p_{k}+p_{k}^{2} \\
= & p_{k}-p_{k}^{2} \\
V\left[I\left(S\left(u_{i}\right) ; k\right)\right]= & p_{k}\left(1-p_{k}\right) .
\end{aligned}
$$$$
=E\left[I\left(S\left(u_{i}\right) ; k\right)\right]-2 p_{k} E\left[I\left(S\left(u_{i}\right) ; k\right)\right]+p_{k}^{2} \text {, como } I\left(S\left(u_{i}\right) ; k\right) \text { é }
$$

Na expressão (3.27) apresenta-se a variância para a $k$-ésima categoria, sendo o produto das proporções entre a frequência da $k$-ésima categoria pela frequência das diferentes $k$ ésimas categorias. Assim, quando se trabalha com variável categórica pode-se obter as $K$ funções indicadoras seguindo a generalização da distribuição de Bernoulli.

Leuangthong et al. (2008, p. 137) descrevem que estas funções indicadoras são variáveis aleatórias mutuamente exclusivas, cumprindo com $\sum_{k=1}^{K} I\left(S\left(u_{i}\right) ; k\right)=1$, confirmando, assim, que a distribuição categórica é um caso especial da distribuição multinomial.

De acordo com Kader e Perry (2007, p. 11) utilizando a expressão (3.27) pode-se obter o coeficiente de diversidade (unalikeability) para todas as $K$ categorias, que é definido como o coeficiente que compara possíveis proporções diferentes, representado por:

$$
\mu_{2}=\sum_{k=1}^{K} p_{k}\left(1-p_{k}\right) \text {. }
$$

\subsubsection{Krigagem Indicatriz}

A metodologia da krigagem indicadora é o interpolador mais comum na estimativa de uma categoria $k$ para uma localização $u$ não amostrada, definido como: 


$$
I_{K I}^{*}\left(S\left(u_{0}\right) ; k\right)=\sum_{i=1}^{n} \lambda_{i} I\left(S\left(u_{i}\right) ; k\right),
$$

onde, $\lambda_{i}$ é o peso assinalado a cada uma das variáveis aleatórias pelas $n$ funções indicatrizes vizinhas ao ponto $u_{0}$.

Seja $A$, um dos valores da variável aleatória $S(u)=k$, onde $k=A$, podendo ser $A=$ $1,2, \ldots, K$, a probabilidade de que o evento $A$ na localização $u$ ocorra, será estimada pelas médias, assim como descrito na equação (3.29), mas substituída pela expressão:

$$
I_{K I}^{*}\left(S\left(u_{0}\right) ; k\right)=P\left(S\left(u_{0}\right) ; k=A\right) .
$$

Também pode-se calcular a incerteza associada com a estimativa da função indicadora $I_{K I}^{*}(3.28)$, como segue:

$$
\mathrm{s}_{0}^{2}\left(S\left(u_{0}\right) ; k\right)=\sum_{i=1}^{n} \lambda_{i}\left[I\left(S\left(u_{i}\right) ; k\right)-I_{K I}^{*}\left(S\left(u_{0}\right) ; k\right)\right]^{2},
$$

que é a variância da interpolação proposta por Yamamoto (2000, p. 491), onde $\sum_{i=1}^{n} \lambda_{i}=1$. Reescrevendo esta expressão tem-se:

$$
\mathrm{s}_{0}^{2}\left(S\left(u_{0}\right) ; k\right)=\sum_{i=1}^{n} \lambda_{i} I^{2}\left(S\left(u_{i}\right) ; k\right)-\left[I_{K I}^{*}\left(S\left(u_{0}\right) ; k\right)\right]^{2} .
$$

Desde que, $\sum_{i=1}^{n} \lambda_{i} I^{2}\left(S\left(u_{i}\right) ; k\right)=\sum_{i=1}^{n} \lambda_{i} I\left(S\left(u_{i}\right) ; k\right)$ a variância de interpolação fica como:

$$
\mathrm{s}_{0}^{2}\left(S\left(u_{0}\right) ; k\right)=I_{K I}^{*}\left(S\left(u_{0}\right) ; k\right)-\left[I_{K I}^{*}\left(S\left(u_{0}\right) ; k\right)\right]^{2}=I_{K I}^{*}\left(S\left(u_{0}\right) ; k\right)\left(1-I_{K I}^{*}\left(S\left(u_{0}\right) ; k\right)\right) .
$$

No caso que $k=A$, a interpolação da variância é

$$
\mathrm{s}_{0}^{2}\left(S\left(u_{0}\right) ; k\right)=P\left[\left(S\left(u_{0}\right) ; k=A\right)\right] P\left[\left(S\left(u_{0}\right) ; k \neq A\right)\right] .
$$

Por outro lado, Yamamoto et al. (2012, p. 148) propõem estimar os $\lambda_{i}$ da KI, equação (3.29), através das equações multi quádricas (3.36), com os pesos $w_{i}$ obtidos da solução do sistema linear de equações, definida por:

$$
\left\{\begin{array}{l}
\sum_{j} w_{j} \phi\left(u_{j}-u_{i}\right)+\mu=\phi\left(u_{0}-u_{i}\right), \text { para } i=1, \ldots, n, \\
\sum_{j} w_{j}=1 .
\end{array}\right.
$$

Como alternativa ao uso da função de variograma substitui-se esta função pelo núcleo multi quádrico (expressão 3.36), quando $k=0$ e $c=0$, de acordo com Hardy (1971, p. 1907). Segundo Franke (1982, p. 195), quando comparado com outros métodos de interpolação, a krigagem indicadora apresenta resultados com maior acurácia em relação às estimativas e 
na visualização suavizada da área ou subsuperfície em estudo, que é representada por:

$$
\phi(u)=\left\{c+\|u\|^{2}\right\}^{(2 k+1) / 2}, \text { para } k=-1,0, \ldots,
$$

onde, $\|u\|$ denota-se como a norma do vetor em $\Re^{2}$ ou $\Re^{3}$ e $c$ constante positiva.

Os pesos $w_{i}$ serão obtidos de (3.35) e substituídos na seguinte equação:

$$
I_{M Q}^{*}\left(S\left(u_{0}\right) ; k\right)=\sum_{i=1}^{n} \hat{w}_{i} I\left(S\left(u_{i}\right) ; k\right) .
$$

Desta forma, utilizando os pesos estimados $\hat{w}_{i}$, calcula-se a incerteza associada a interpolação, definida por:

$$
\hat{\mathrm{s}}_{0}^{2}\left(S\left(u_{0}\right) ; k\right)=\sum_{i=1}^{n} \hat{w}_{i}\left[I\left(S\left(u_{i}\right) ; k\right)-I_{M Q}^{*}\left(S\left(u_{0}\right) ; k\right)\right]^{2} .
$$

Utilizando a função multi quádrica, a variância global será obtida substituindo a expressão (3.38) em (3.28), assim como:

$$
\hat{\mathrm{s}}^{2}=\sum_{k=1}^{K} \hat{\mathrm{s}}_{0}^{2}\left(S\left(u_{0}\right) ; k\right)=\sum_{k=1}^{K} \sum_{i=1}^{n} \hat{w}_{i}\left[I\left(S\left(u_{i}\right) ; k\right)-I_{M Q}^{*}\left(S\left(u_{0}\right) ; k\right)\right]^{2},
$$

equivalentemente, utilizando a krigagem indicatriz substitui-se a expressão (3.33) em (3.28), tem-se

$$
\hat{\mathrm{s}}^{2}=\sum_{k=1}^{K} I_{K I}^{*}\left(S\left(u_{0}\right) ; k\right)\left(1-I_{K I}^{*}\left(S\left(u_{0}\right) ; k\right)\right),
$$

ou quando $p_{k}$ é conhecido, pode ser representado por:

$$
\mathrm{s}^{2}=\sum_{k=1}^{K} p_{k}\left(1-p_{k}\right)
$$

Neste trabalho usa-se a função indicadora para calcular a variância de interpolação, definida por Yamamoto (2000, p. 491, expressão 3.31). Desta forma, considera-se a sugestão dada por Yamamoto et al. (2012, p. 151) onde se utiliza a equação (3.27) para encontrar incertezas com variância de interpolação entre o intervalo 0.20 a 0.25 . Portanto, assume-se também este intervalo para identificar incertezas através da metodologia multi-ponto para as $K$ categorias.

\subsection{Terminologia geoestatística multi-pontos}

Segundo Strebelle (2002, p. 3) define-se o processo geoestatístico estocástico considerando um atributo $S$ tomando os $K$ possíveis estados $\left\{s_{k}, k=1, \ldots, K\right\}$. Onde $S$ é uma variável 
aleatória discreta dentro de $K$ classes. A seguinte terminologia será usada (Strebelle, 2002, p. 3):

- Os dados do evento $d_{n}$ de tamanho $n$, será centrado para a localização $u$ a ser simulado e será constituído por:

- O dado da geometria definida por $n$ vetores $\left\{h_{\alpha}, \alpha=1, \ldots, n\right\}$;

- Os valores do dado $\left\{s\left(u+h_{\alpha}\right), \alpha=1, \ldots, n\right\}$.

O valor central dos dados do evento é um valor desconhecido a ser avaliado, denotado como $s(u)$.

- Um padrão de dados $\tau_{n}$, compreende apenas a geometria dos dados anteriores. Um subpadrão de $\tau_{n^{\prime}}$, é um padrão constituído por qualquer subconjunto de vetores $n^{\prime}$ de $\tau_{n}, \operatorname{com} n^{\prime} \leq n$.

Os dados do evento $d_{n}$ é associado com um padrão geométrico $\tau_{n}$.

- De forma estocástica, os $K$ possíveis estados de uma variável aleatória $S(u)$, são caracterizados por sua função de probabilidade condicional (fpc), denota-se como:

$$
\operatorname{Prob}\left\{S(u)=s_{k} \mid d_{n}\right\}=f\left(u ; s_{k} \mid d_{n}\right), \quad \forall k=1, \ldots, K
$$

Sabe-se que, a fpc para quaisquer dado do evento $d_{n}$ são suficientes para realizar a simulação estocástica de Monte Carlo da variável aleatória $S(u)$. Observa-se que, para cada $n$, dado a variável $S(u)$, esta pode tomar diferentes valores de $K$, totalizando $K^{n}$.

Para uma melhor concepção da nomenclatura utilizada, representa-se na Figura 3.2 a configuração da variável $S(u)$.

\subsection{Geoestatística multi-pontos}

A geoestatística multi-pontos (MPS) caracteriza a relação entre pontos finitos distribuídos espacialmente, sendo que a MPS permite a reprodução de padrões de heterogeneidade curvilínea e relações de ordenação. Porém, se a metodologia MPS é aplicada para dois pontos, este cai sobre a estatística de variogramas e semi-variograma, sendo portanto uma medida de continuidade espacial linear. No entanto, existem diferentes tipos de MPS:

1. MPS com função de conectividade.

2. MPS com função de lacunaridade.

3. MPS com função de probabilidade condicional (fpc). 


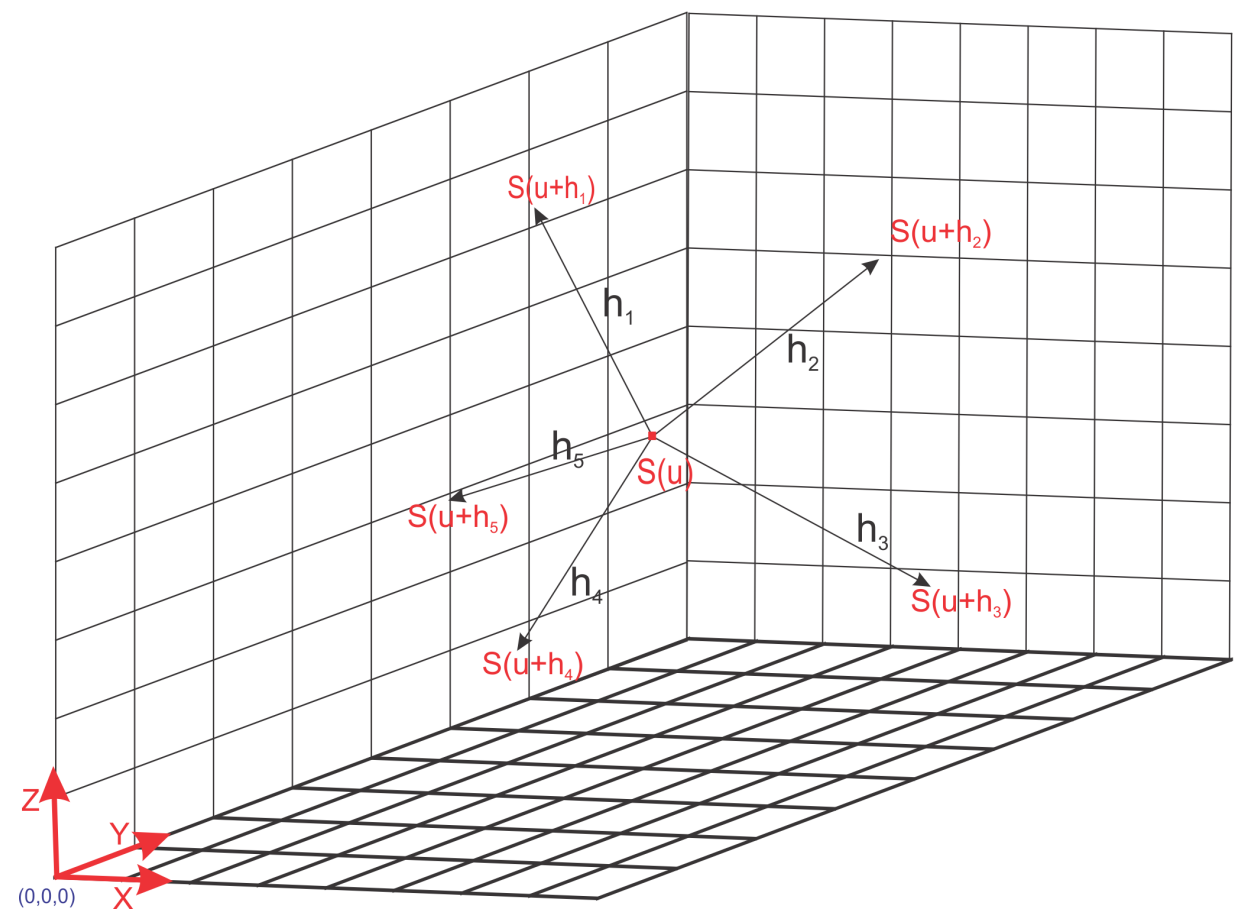

Figura 3.2: Configuração da variável $S(u)$ condicionada.

A MPS com função de conectividade obtida pela imagem a ser estudada permite uma análise qualitativa da caracterização geométrica do corpo (subsuperfície), tudo isto relacionado com a probabilidade de dois pixels arbitrários separados por uma distância $u$ pertencendo à grade especificada. A MPS com função de lacunaridade é utilizada para a caracterização granulométrica e a porosidade dos materiais. Aplicando na mineração esta função servirá para uma caracterização de rochas.

No item 3.7 será amplamente discutido a relação da geoestatística multi-pontos com a função de probabilidade condicional.

Justifica-se a utilização da MPS por ser uma solução mais eficiente para as limitações inerentes das tradicionais técnicas geoestatísticas baseadas na estatística multi-pontos, por meio da função variograma.

Este foi o ponto inicial ao desenvolvimento da geoestatística multi-pontos dado por Deutsch (1992) e Guardiano e Srivastava (1993). Mais tarde, o desenvolvimento de um algoritmo prático de simulação para MPS, desenvolvido por Strebelle (2000), generalizou a simulação MPS para identificar as diversas camadas que compunham determinado reservatório.

\subsection{Definição da geoestatística multi-ponto sobre a equação estendida}

Considere o modo estocástico ao atributo $S$ tomando possíveis estados de $K\left\{s_{k}, k=\right.$ $1, \ldots, K\}$ onde avalia-se a função de probabilidade da variável $S(u)$ condicionado à ocorrên- 
cia do estado $S$, desta forma Strebelle (2002, p. 5) define:

$$
A_{k}=\left\{\begin{array}{cc}
1 & \text { se } S(u)=s_{k} \\
0 & \text { caso contrário(c.c.). }
\end{array}\right.
$$

Similarmente, para $D$ tem-se uma variável aleatória associada às ocorrências dos eventos $d_{n}$, constituídos por $n$ dados condicionados com $S\left(u_{\alpha}\right)=s_{\alpha}$, onde $\alpha=1, \ldots, n$, considerando conjuntamente:

$$
D=\left\{\begin{array}{cc}
1 & \text { se } S\left(u_{\alpha}\right)=s_{\alpha}, \forall \alpha=1, \ldots, n . \\
0 & \text { c.c. }
\end{array}\right.
$$

$D$ pode ser descomposto pelo produto das variáveis aleatórias associadas para cada dado condicionado:

$$
D=\prod_{\alpha=1}^{n} A_{\alpha}, \operatorname{com} A_{\alpha}=\left\{\begin{array}{cc}
1 & \text { se } S\left(u_{\alpha}\right)=s_{\alpha} \\
0 & \text { c.c. }
\end{array}\right.
$$

Segundo Strebelle (2000, p. 7) no limite, se toda estatística $(n+1)$-pontos relevantes para $A_{k}$ e dado o evento $D$ disponíveis, a probabilidade condicional exata estaria dada pela expressão da krigagem simples (KS):

$$
\operatorname{Prob}\left\{A_{k}=1 \mid D=1\right\}=E\left\{A_{k}\right\}+\lambda[1-E\{D\}]
$$

onde:

- $D=1$ é o evento do dado observado,

- $E\{D\}=\operatorname{Prob}\left\{\prod_{\alpha=1}^{n} A_{\alpha}=1\right\}=E\left\{\prod_{\alpha=1}^{n} A_{\alpha}=1\right\}$ é a probabilidade condicionada aos dados do evento que ocorre,

- $E\left\{A_{k}\right\}=\operatorname{Prob}\left\{S(u)=s_{k}\right\}=p_{k}$ é a probabilidade a priori para o estado $u$ sendo igual a $s_{k}$ (não conhecida). Esta probabilidade é conhecida a priori aos dados do evento $D=1$.

Assim, pelo teorema de Bayes não há necessidade de estender a equação da Krigagem simples (3.46) para $(n+1)$-pontos, como demonstrado pelo desenvolvimento realizado por Strebelle (2000, p. 7), desde uma expressão definida desde a equação da Krigagem simples (dois pontos) até chegar a estatística $(n+1)$-pontos, quando aplicamos as expressões (3.44) e (3.45) na equação estendida.

Desta forma, mostra-se, segundo Strebelle (2000, p. 7, expressões (1) e (2)) a equação estendida para dois pontos:

$$
\operatorname{Prob}\left\{A_{k}=1 \mid D=1\right\} \simeq E\left\{A_{k}\right\}+\sum_{\alpha=1}^{n} \lambda_{\alpha}\left[1-E\left\{A_{\alpha}\right\}\right]
$$


com:

- $A_{\alpha}=1$ sendo valor indicador para os valores do dado $S_{\alpha}$ na localização $u_{\alpha}$,

- $E\left\{A_{\alpha}\right\}=\operatorname{Prob}\left\{S\left(u_{\alpha}\right)=s_{\alpha}\right\}=p_{\alpha}$ : probabilidade a priori para o estado $s_{\alpha}$,

- $E\left\{A_{k}\right\}=\operatorname{Prob}\left\{S(u)=s_{k}\right\}=p_{k}$,

e para três pontos:

$$
\operatorname{Prob}\left\{A_{k}=1 \mid D=1\right\} \simeq E\left\{A_{k}\right\}+\sum_{\alpha=1}^{n} \lambda_{\alpha}\left[1-E\left\{A_{\alpha}\right\}\right]+\sum_{\beta=1}^{\left(\begin{array}{c}
n \\
2
\end{array}\right)} \mu_{\beta}\left[1-E\left\{B_{\beta}\right\}\right],
$$

onde, $\left(\begin{array}{l}n \\ 2\end{array}\right)$ é a combinação existente de dois pontos localizados fora do total de $n$ amostras, e $B_{\beta}$ é o resultado correspondente ao produto dos indicadores, por exemplo, $B_{\beta}=A_{\beta_{1}} A_{\beta_{2}}$ cuja realização $b_{\beta}=1$ corresponde ao dado do evento de dois pontos: $\left\{S\left(u_{\beta_{1}}\right)=s_{\beta_{1}}\right.$ e $\left.S\left(u_{\beta_{2}}\right)=s_{\beta_{2}}\right\}$.

Desta forma, assim como definido anteriormente as variáveis $A_{k}$ e $D$ pelo teorema de Bayes, pode-se obter por definição a seguinte expressão (Strebelle, 2000, p. 8, expressão 5):

$$
\operatorname{Prob}\left\{A_{k}=1 \mid D=1\right\}=\frac{\operatorname{Prob}\left\{A_{k}=1, D=1\right\}}{\operatorname{Prob}\{D=1\}}=\frac{E\left\{A_{k} D\right\}}{E\{D\}} .
$$

A relação da probabilidade condicional, assim como definida em (4.1) identifica uma solução exata por definição. A solução exata obtida em (3.49) para as chamadas estatísticas $(n+1)$-pontos, vai muito além das estatísticas tradicionais de dois pontos, variogramas ou modelos de covariâncias (Strebelle, 2000, p. 8). Normalmente, não existe a esperança de tais geoestatísticas multi-pontos para poder inferir os valores reais (superfície ou subsuperfície). Assim, realiza-se a leitura de uma ou mais imagens de treinamento. Pode-se digitalizar imagens, tanto para as probabilidades de dois pontos exigidos pela expressão tradicional KS em (3.47) e (3.48), quanto para as probabilidades requeridas para $(n+1)$-pontos geoestatísticos, necessária para a expressão exata definida em (3.46). Note que, se repassamos as estatísticas para a formação da imagem, a expressão (3.46) é única (exata) considerando a decisão de estacionariedade, que permite exportar estatísticas desde a imagem de treinamento para a atual região em estudo de interesse.

\subsection{Digitalizando a imagem de treinamento}

De acordo com a decisão a priori de estacionariedade (permissão para a exportação), as imagens de treinamento são utilizadas para obter as expressões da probabilidade condicional do numerador e denominador descritas em (3.49), assim como descrito por Strebelle (2002, p. 6) define-se: 
- O denominador $\operatorname{Prob}\{D=1\}=\operatorname{Prob}\left\{S\left(u_{\alpha}\right)=s_{\alpha}, \alpha=1, \ldots, n\right\}$ pode ser inferido pela contagem de números de $c$ réplicas condicionadas ao dado do evento $d_{n}=\left\{S\left(u_{\alpha}\right)\right.$ $\left.=s_{\alpha}, \alpha=1, \ldots, n\right\}$ na imagem de treinamento. A repetição deve ter a mesma configuração geométrica e os mesmos valores fornecidos em $s_{\alpha}$.

- O numerador $\operatorname{Prob}\left\{S(u)=s_{k}\right.$ e $\left.S\left(u_{\alpha}\right)=s_{\alpha}, \alpha=1, \ldots, n\right\}$ é obtido por contagem do número de $c_{k}$ repetições, entre os $c$ anteriores, associado a um valor central $S(u)$ igual a $s_{k}$.

A probabilidade condicional, necessária, é então, identificada para a proporção de formação $\frac{c_{k}}{c}$ :

$$
p\left(u ; s_{k} \mid d_{n}\right)=\operatorname{Prob}\left\{A_{k}=1 \mid D=1\right\}=\operatorname{Prob}\left\{S(u)=s_{k} \mid d_{n}\right\} \simeq \frac{c_{k}}{c},
$$

onde, $\sum_{k=1}^{K} c_{k}=c$.

Neste caso, o limite de uma única equação estendida (3.46) é absolutamente simples na medida em que se reduz a própria definição da probabilidade condicional, que é obtida pela utilização da imagem de treinamento condicionado a um template de busca.

\subsection{Composição da geoestatística multi-pontos}

Um importante aspecto de abordagem expressado na seção (3.5) relacionada com a geoestatística multi-pontos, deverá ser mantida para a simulação condicional em qualquer e particular célula $u$, em 2D (pixels) ou se for em 3D (voxels).

Considerando novamente o caso da variável discreta $S(u)$ e as suas distribuições de probabilidades condicionais $\operatorname{Prob}\left\{S(u)=s_{k} \mid d_{n}\right\}=p\left(u ; s_{k} \mid d_{n}\right), \forall k=1, \ldots, K$, será necessário relacionar a quantidade de dados vizinhos que definiram o condicionamento dos dados do evento em $d_{n}$ na imagem de treinamento (Strebelle, 2002, p. 7). Quanto mais dados vizinhos, maior o tamanho de $n$ em $d_{n}$ através de um template de busca, quanto mais específico o evento dos dados de $d_{n}$, menos repetições serão encontradas sobre a imagem de treinamento, por inferência da probabilidade condicional correspondente a $p\left(u ; s_{k} \mid d_{n}\right)$. Consequentemente, quando a MPS for muito específica, a distribuição de probabilidade $p\left(u ; s_{k} \mid d_{n}\right), \forall k=1, \ldots, K$, acaba sendo difícil reter na imagem de treinamento as probabilidades condicionais, e assim, exportá-la para a superfície ou subsuperfície de interesse pode ser inadequada, porque poderia estar exportando padrões idiossincráticos da imagem de treinamento em vez da sua essência (Strebelle, 2002, p. 7).

Strebelle (2002, p. 7) propõe uma alternativa para diminuir o tamanho dos dados vizinhos até os dados do evento $d_{n}$ retidos, sejam os mais frequentes da imagem de treinamento, fixando um $c_{\text {min }}$ entre 10 e 20. Isto, permitirá uma correspondência da função de probabilidade $\left\{p\left(u ; s_{k} \mid d_{n}\right), \forall k=1, \ldots, K\right\}$ com a característica essencial da imagem de treinamento, que é comumente encontrada. 
Uma vez que os dados do evento condicionado $d_{n}$ são definidos, a segunda questão é relacionar qual geoestatística multi-pontos pode ser considerada para aproximação da distribuição de probabilidade condicional $\left\{p\left(u ; s_{k} \mid d_{n}\right), \forall k=1, \ldots, K\right\}$ (Strebelle, 2002, p. 7). Assim, como na abordagem da geoestatística tradicional (estatística de 2 pontos), onde se faz inferência a partir de dados reais da subsuperfície (expressão 3.47), a geoestatística multi-pontos se baseia nos dados amostrados e no comportamento anterior conhecido para realizar sua inferência. Uma abordagem menos objetiva consistiria em realizar através da geoestatística de dois pontos uma imagem de treinamento (por exemplo, variograma), isto é comumente feito em aplicações para petróleo inferindo variogramas horizontais.

Quanto maior a dimensão de $n$ das geoestatísticas multi-pontos, a imagem de treinamento fica inviável de ser trabalhada e, portanto, menos exportável para subsuperfície estudada, voltando ao dilema qual seria o $n$ ótimo. Note, no entanto, que a objetividade de se basear apenas nos dados reais da área ou subsuperfície de interesse interrompe o modelo numérico usado para construir o fenômeno, o que implica em todos os modelos da geoestatística multipontos, estes não são apenas dados fornecidos, pois eles participam no condicionamento da área ou subsuperfície estudada.

A abordagem de simulação proposta a seguir consiste em inferir a geoestatística multipontos através da imagem de treinamento, reduzindo, se necessário, o tamanho para $n-m$ dados condicionados, onde $n \geq m$. Uma grande vantagem desta proposta é que o resultado da probabilidade condicional (3.49) é, por definição, admissível, pois não é exposta a problemas de quaisquer ordem associados às expressões limitadas da KS do tipo (3.47) e (3.48). A consistência (sem ordem de relação) não é sinônimo de precisão. Sendo então, a expressão de probabilidade (3.49) usada exatamente para a imagem de treinamento, ela pode ser relevante para os dados reais o qual é aplicado. 


\section{Capítulo 4}

\section{Algoritmo}

Esta seção apresenta a descrição do algoritmo que será utilizado para o desenvolvimento do aplicativo, com base no SNESIM (Remy et al.,2009, p. 171, algoritmo 8.11).

\subsection{Simulação da equação estendida}

O algoritmo de simulação apresentado a seguir será inicialmente desenvolvido para variáveis aleatórias discretas, porém pode ser estendido para variáveis aleatórias contínuas utilizando o processo de discretização para $K$ classes.

Este paradigma está baseado no algoritmo da simulação sequencial, em que cada valor simulado torna-se um valor referencial condicionado para o nó ou célula ainda não simulada e que posteriormente são utilizados em sequência, segundo Goovaerts (1997, p. 393).

Guardiano e Srivastava (1993, p. 136) propuseram uma varredura repetitiva em toda a imagem de treinamento para cada nó não amostrado, inferindo a distribuição de probabilidade condicional local, pois, este procedimento utilizava excessivo tempo de processamento computacional, especialmente quando consideramos grandes imagens de treinamento ou quando gerava um grande número de realizações para cada um dos nós. A implementação do algoritmo consiste primeiramente em tabular todas as fpcs, e realizar os seguintes passos segundo Remy et al. (2009, p. 170):

1. Um modelo com $n$ variáveis associadas a seu correspondente vetor, onde cada variável pode tomar os $K$ possíveis valores e generalizando, tem-se $K^{n}$ resultados possíveis.

2. No método da simulação sequencial, o padrão dos dados condicionados geometricamente depende da localização dos nós (células) previamente simulados, consequentemente, os nós serão visitados ao longo do caminho aleatório, quando houver mudança no padrão de um nó para outro.

A proposta da implementação do algoritmo deste trabalho é reproduzir os resultados obtidos por Guardiano e Srivastava (1993, p. 136, equação (3.49)) e por Strebelle (2002, 
p. 9 e 10, itens (4.1 a 6) da seção 4.2) em um ambiente de programação acessível para a comunidade científica, através do software R.

A primeira etapa (Passo 1) do algoritmo é realizada de forma que os valores de $S(u)$ e suas condicionantes são capturadas através da imagem de treinamento, definida inicialmente de tal forma que os valores do template $\tau_{n}$ são constituídos por vetores de tamanho $n$, assim como $\left\{h_{\alpha}, \alpha=1, \ldots, n\right\}$, identificando que as $n$ localizações vetoriais $u_{\alpha}=u+h_{\alpha}$, $\alpha=1, \ldots, n$, correspondem as $n$ posições dos nós da célula mais próxima de $u$, assim como mostrado a Figura (3.2). Especificamente, pode-se observar o template utilizado nas Figuras (4.1) e (4.2) representadas em três dimensões $\Re^{3}(3 \mathrm{D})$. Seja, $\tau_{n^{\prime}}=\left\{h_{\alpha}, \alpha=1, \ldots, n^{\prime}\right\}$, o submodelo constituído pelos primeiros $n^{\prime}$ de $\tau_{n}$; as $n^{\prime}$ localizações $u+h_{\alpha}, \alpha=1, \ldots, n^{\prime}$, são as $n^{\prime}$ posições dos nós da célula mais próximos de $u$ (Figura 4.3).

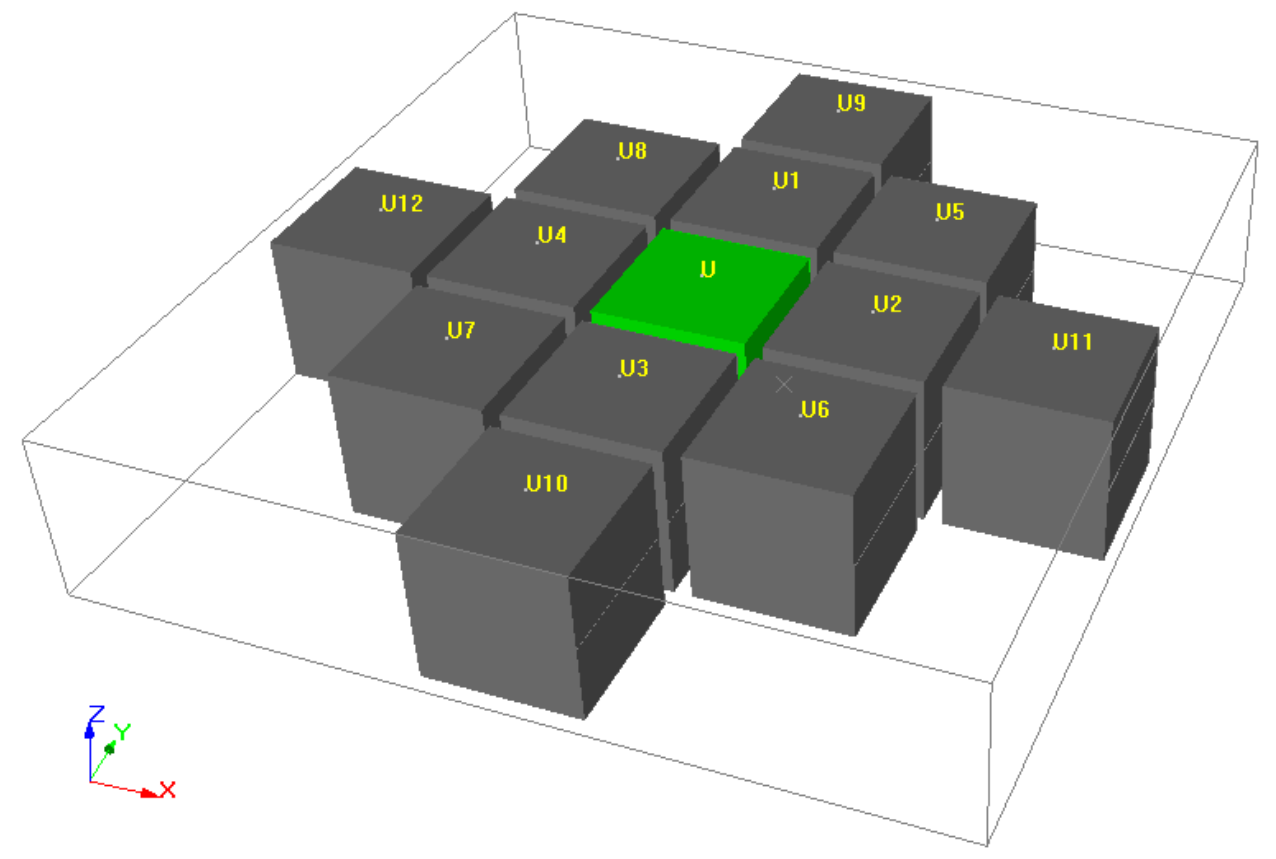

Figura 4.1: Configuração da variável $S(u)$ condicionada a um template $\tau_{n} \operatorname{com} n=12$.

Para qualquer $n^{\prime}$, dado o evento $d_{n^{\prime}}$ denotado por $c\left(d_{n^{\prime}}\right)$, o número de $d_{n^{\prime}}$ repetições na imagem de treinamento através de $c_{k}\left(d_{n^{\prime}}\right)$ é o número de repetições entre os $c\left(d_{n^{\prime}}\right)$ anteriores cujo valor central é $S_{k}$, como por exemplo quando $n^{\prime}=10$, mostrado na Figura (4.3).

Strebelle (2002, p. 6) propôs armazenar em uma estrutura de dados com apenas os valores $c_{k}\left(d_{n^{\prime}}\right)$ correspondentes aos dados do evento $d_{n^{\prime}}$ associados aos submodelos $\tau_{n^{\prime}}, n^{\prime}=1, \ldots, n$. Desta forma, pelo menos uma repetição será encontrada na imagem de treinamento. O conhecimento desses números $c_{k}\left(d_{n^{\prime}}\right)$ permite recuperar qualquer fpc existente na imagem de treinamento, desde que a sua configuração de dados condicionado esteja incluída em $\tau_{n^{\prime}}$.

O número total de repetições do evento $d_{n^{\prime}}$ associado com $\tau_{n^{\prime}}$, garante que pelo menos uma repetição na imagem de treinamento escaneada pelo submodelo $\tau_{n^{\prime}}$ será encontrada. Segundo Strebelle (2002, p. 9), o número de buscas no nó da árvore será limitado por $n$ e desta forma, o algoritmo não requererá maior quantidade de memória RAM para os dados 


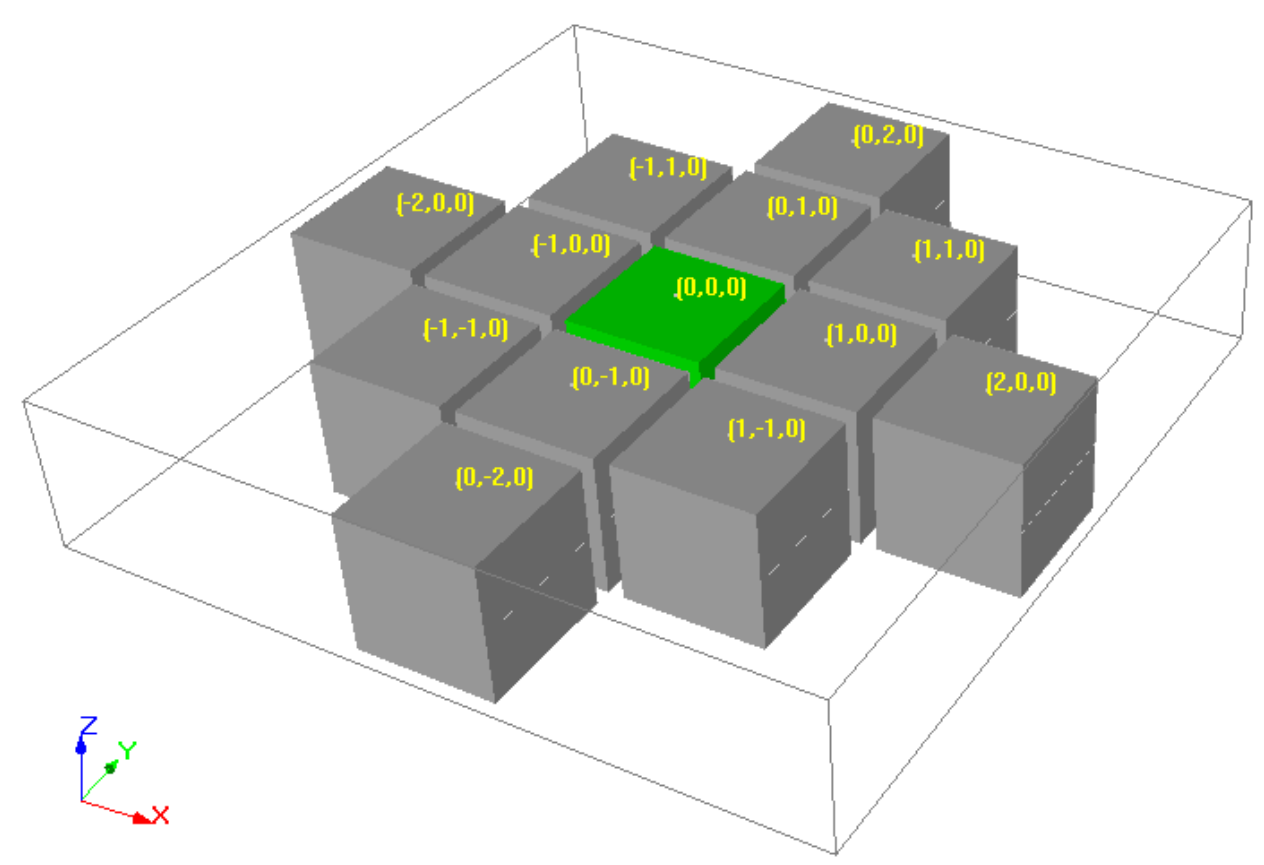

Figura 4.2: Configuração da variável $S(u)$ condicionada a um template $\tau_{n} \operatorname{com} n=12$, assim como utilizado no programa.

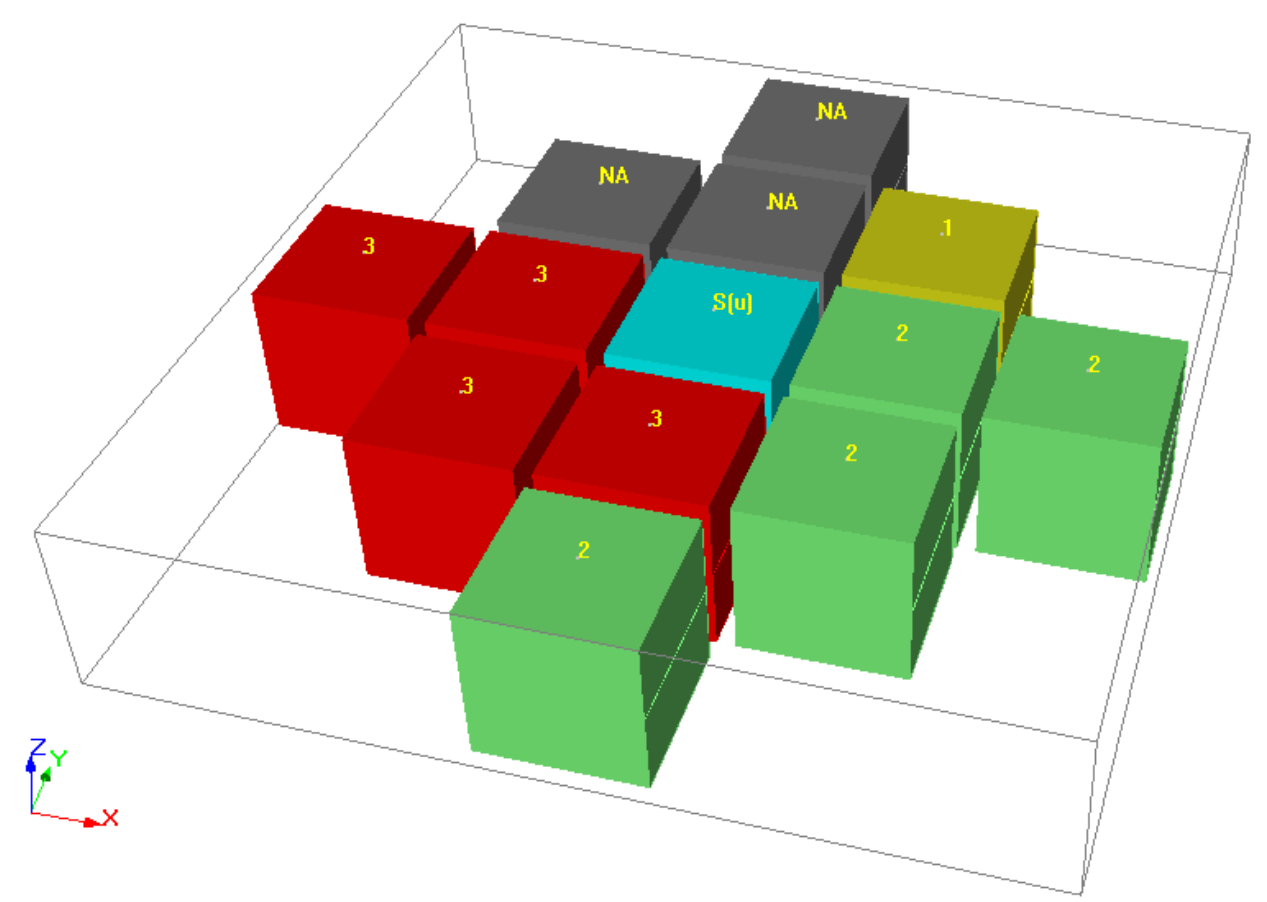

Figura 4.3: Configuração da variável $S(u)$ condicionada a um template $\tau_{n^{\prime}}$ num caso particular quando $n^{\prime}=10$, assim como utilizado no programa. 
do template $\tau_{n}$ com um número razoável de nós $n$ retidos. Por exemplo pode-se definir ao menos 100 nós, que já é um número grande de células.

A descrição dos detalhes da programação e otimização da demanda de memória para obter o número de estruturas de dados (árvore de busca) para fornecimento e classificação de números $c_{k}\left(d_{n}\right)$, podem ser vista com maiores detalhes nos capítulos posteriores. A construção de tal árvore de busca requer o escaneamento da imagem de treinamento uma única vez para a imagem de simulação. Em cada nó não amostrado, o local da distribuição da probabilidade condicional será recuperado diretamente a partir da árvore de busca e a imagem de treinamento não precisará ser escaneada novamente.

\subsection{Algoritmo de simulação}

Apresentam-se as principais etapas do algoritmo de simulação SNESIM, segundo Strebelle (2000, p. 12 e 13) e Remy et al. (2009, p. 171) e comentários a seguir:

1. Primeiramente, o template de busca é escolhido e a imagem de treinamento é escaneada para construir a árvore de probabilidades condicionais; dado um template $\tau_{n}$ constituído pelos $n$ vetores $\left\{h_{\alpha}, \alpha=1, \ldots, n\right\}$, tal que as $n$ posições $u_{\alpha}=u+h_{\alpha}, \alpha=1, \ldots, n$, correspondam aos $n$ nós da célula que são as posições mais próximas de $u$. Os $n$ vetores $h_{\alpha}$ são ordenados de forma crescente pelo valor do seu módulo (Figura 4.1) e também é possível escolher outros templates de busca (Figura 4.5). Desta forma, escolheu-se para estudo de caso o templates de busca mostrado na Figura 4.5 (V), realizando:

$$
\underbrace{\left\|h_{1}\right\|}_{u_{1}=u+h_{1}} \leq \underbrace{\left\|h_{2}\right\|}_{u_{2}=u+h_{2}} \leq \ldots \leq \underbrace{\left\|h_{n}\right\|}_{u_{n}=u+h_{n}}
$$

onde $\|\cdot\|$ é a norma do vetor ${ }^{1}$, de maneira que obtém-se as $n$ posições do vetor $\tau_{n}$.

2. Atribui-se os dados da amostra original para seus nós da célula (voxel) e define-se um caminho aleatório sobre a localização mais próxima das amostras, passando em todos os nós não amostrados que serão estimados dentro das células na área em estudo.

3. Para cada posição $u$ não amostrada mantém-se as $n$ posições dos pontos nodais $u_{\alpha}=$ $u+h_{\alpha}, \alpha=1, \ldots, n$, dos dados do template $\tau_{n}$ e apenas $n^{\prime}(\leq n)$ posições ativas informadas pelos dados para associar na árvore de probabilidades mapeadas pela imagem de treinamento. A ativação do evento é ilustrada nas Figuras (4.1) e (4.3). Seja $d_{n^{\prime}}$ o evento dos dados constituído por aqueles $n^{\prime}$ dados condicionados que é recuperado a partir da árvore de busca, o número $c_{k}\left(d_{n^{\prime}}\right)$ de treinamentos com as $d_{n^{\prime}}$ repetições para a qual o valor central em $u$ é igual para $s_{k}, k=1,2, \ldots, K$. Para obter uma melhor inferência do local das fpc, propõe-se primeiramente verificar a existência das

\footnotetext{
${ }^{1}$ No caso $3 \mathrm{D}$ a norma do vetor $h=(x, y, z)$ é $\sqrt{x^{2}+y^{2}+z^{2}}$.
} 
$d_{n^{\prime}}$ repetições desse evento associado ao espaço amostral em $d_{n^{\prime}}$ da imagem de treinamento com $c\left(d_{n^{\prime}}\right)$ repetições, e se não existir esse evento no espaço amostral criado pelo template de busca nas $n^{\prime}$ primeiras estatísticas multi-pontos, diminuímos as $n^{\prime}$ estatísticas multi-pontos em $n^{\prime}-1$ mais próximos de $u$ e assim sucessivamente até encontrar pelo menos uma estatística suficiente para realizar a estimativa para $S(u)$. Diferentemente da proposta de Strebelle (2002, p.9, no item 3.) que utiliza as probabilidades marginais a priori $p_{k}$, o trabalho desenvolvido nesta tese as substitui pelas as probabilidades condicionais $p\left(u ; s_{k} \mid d_{n}\right)$ quando não existir o evento amostral ocasionado pelas $d_{n^{\prime}}$ estatísticas multi-pontos.

4. Extrair um valor $s$ simulado para o nó $u$ através da função de probabilidade condicional $p\left(u ; s_{k} \mid d_{n}\right)$, e que o valor $s$ simulado seja adicionado aos dados para ser usado pela simulação condicionada em todos os outros nós das células e guarda-se a fpc para estudos posteriores.

5. Mover para o próximo nó ao longo do caminho aleatório e repetir os passos 3 e 4.

6. Repetir até todos os nós do modelo de células sejam simulados. Uma imagem estocástica será gerada que representará a primeira realização do processo estocástico. Repetir o processo a partir do passo 2 com outro arranjo aleatório para as próximas realizações.

\subsection{Condicionamento dos dados observados}

Segundo Strebelle (2000, p. 13), duas condições devem ser atendidas para garantir o condicionamento adequado aos dados observados (dados reais):

1. Os dados observados devem ser reproduzidos exatamente em suas localizações.

2. Nós aproximados por qualquer localização do dado observado tem maior probabilidade e, portanto, menor variância condicional. Mais precisamente, a variância dos $L$ valores simulados $\left\{s^{l}, l=1, \ldots, L\right\}$ no nó $u$, deve diminuir a variância à medida que o dado observado se aproxima da localização $u_{\alpha}$.

Segundo Strebelle (2000, p. 14), realoca-se os dados da amostra para a malha de simulação e a fixação de seus valores garante o condicionamento para a simulação dos nós não amostrados. Como a localização $u$ se aproxima dos dados observados na localização $u_{1}$, o treinamento da distribuição de probabilidade $\left\{p\left(u ; s_{k} \mid d_{n}\right), k=1, \ldots, K\right\}$ aproxima-se em um único núcleo a distribuição para $S(u)=s_{k}$ se $S\left(u_{1}\right)=s_{k}$. De fato, pela continuidade espacial da imagem de treinamento do valor central $s(u)$ de qualquer modelo de dados com $u_{1}$ perto de $u$ será cada vez mais frequente no mesmo estado, como o valor do dado condicionado $s\left(u_{1}\right)$. A menor escala da continuidade espacial da imagem de treinamento é passada para a 
realização simulada através das proporções de treinamento. Aqui como o nó que está sendo simulado, ele se aproxima para o valor observado e sua variância condicional diminui.

\subsection{Introdução da simulação multi-células}

A vizinhança dos dados condicionados definidos pelo template de busca dos dados $\tau_{n}$ retidos, não deve ser muito pequena, de certa forma, grandes estruturas de escalas da imagem de treinamento não serão reproduzidas, segundo Strebelle (2002, p. 8). Por outro lado, Remy et al. (2009, p. 170) propõem uma expressão relacionada a demanda de memória sobre um modelo de dados $\tau_{n}$ na metodologia multi-pontos, de tal forma que o tempo da demanda de memória para construção da árvore de probabilidades condicionais será proporcional ao número de níveis, quanto maior os níveis na árvore de busca, maior o número de nós, portanto, ainda maior será a demanda em tempo de processamento. A expressão é descrita como:

$$
\text { Demanda de memória } \leq \sum_{j=1}^{J} \min \left(K_{j}, N_{\tau_{n}}\right)
$$

onde $N_{\tau_{n}}$ é o número total de nós nas células da imagem de treinamento e $K_{j}$ o número de categorias na $j$-ésima célula da imagem de treinamento.

Uma solução para capturar estruturas em grande escala, enquanto se considere um template de busca de $\tau_{n}$ incluindo um número razoavelmente menor de nós das células, é a abordagem multi-células, inicialmente proposto por Gomez-Hernandez (1991, p. 109) e por Deutsch e Journel (1992, p. 124-125, p. 187) e desenvolvida por Tran (1994, p. 1165). Ilustrase o conceito de multi-células na Figura (4.4), onde observa-se a expansão do arranjo do template de busca $\tau_{n}$ de (I) passando por (II), (III) e (IV) de forma que, se atinja estruturas maiores.

।

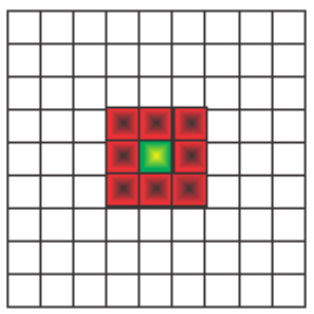

II

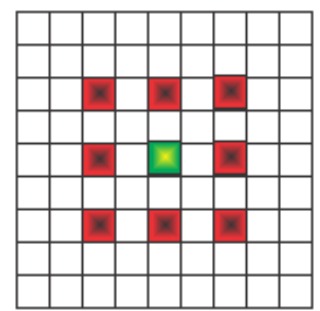

III

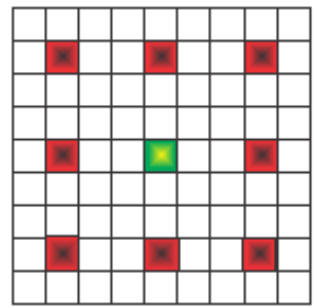

IV

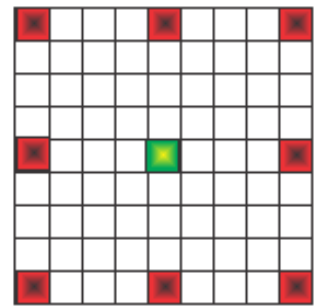

Figura 4.4: Estruturas de template de busca, configurações (I), (II), (III) e (IV), onde, captura-se informação cada vez mais abrangente.

Uma árvore de busca precisa ser construída pelas células de simulação, considerando que $n_{g}$ permanece constante, igual a $n$ ou não. Quando a $g$-ésima célula de simulação é completada seus valores simulados são mantidos como dados a serem usados para o condicionamento do próximo nó, sendo, desta forma, uma simulação mais fina. 
As primeiras amostras originais requerem de um condicionamento adequado para os nós mais próximos da célula de simulação sejam atingidos pelo passeio aleatório. Uma vez que todos os nós da malha atual são simulados, os dados da amostra são transferidos para seus locais originais. Os dados da amostra removidos dos nós da malha são simulados posteriormente seguindo os nós de uma malha mais abrangente. E uma representação em 3D dos templates de busca, tem-se na Figura (4.5).

\subsection{Reprodução da função de probabilidade condicional}

A função de probabilidade descrita por $\left\{p_{k}, k=1, \ldots, K\right\}$ é a probabilidade não condicionada, identificada na aplicação da metodologia multi-pontos e é definida como a probabilidade a priori, no nível 0 na árvore de probabilidades condicionais, segundo Strebelle (2000, p. 15, expressão 6).

Desta maneira, espera-se, por definição de probabilidade condicional, não reproduzir a probabilidade a priori na imagem de saída (imagem simulada) ao finalizar o processo da aplicação da metodologia multi-ponto, em contraste a proposta de Strebelle (2000, p. 15). $\mathrm{Na}$ realidade, esse autor ajusta e rearranja as probabilidades condicionais iterativamente, de forma que perturba os resultados da imagem simulada.

Por definição de probabilidades condicionais e especificamente no caso discreto as probabilidades a priori não serão mais reproduzidas na imagem de saída. Veja-se a definição de probabilidade condicional em (4.1), pois ao trabalhar com probabilidades condicionais diminui-se o espaço amostral, de forma que a chance de um evento acontecer diminui, pois o espaço amostral diminuí:

Definição 4.1 Probabilidade condicional: Sejam $S(u)$ e $d_{n}$ duas variáveis aleatórias, a probabilidade condicional é expressa pela função de $S(u)$ dado que $d_{n}=\tau_{n}$ e é definida para todo $d_{n}$ tal que $P\left\{d_{n}=\tau_{n}\right\}>0$ como:

$$
P\left\{S(u)=s_{k} \mid d_{n}=\tau_{n}\right\}=\frac{P\left\{S(u)=s_{k}, d_{n}=\tau_{n}\right\}}{P\left\{d_{n}=\tau_{n}\right\}},
$$

onde $k=1,2, \ldots, K$.
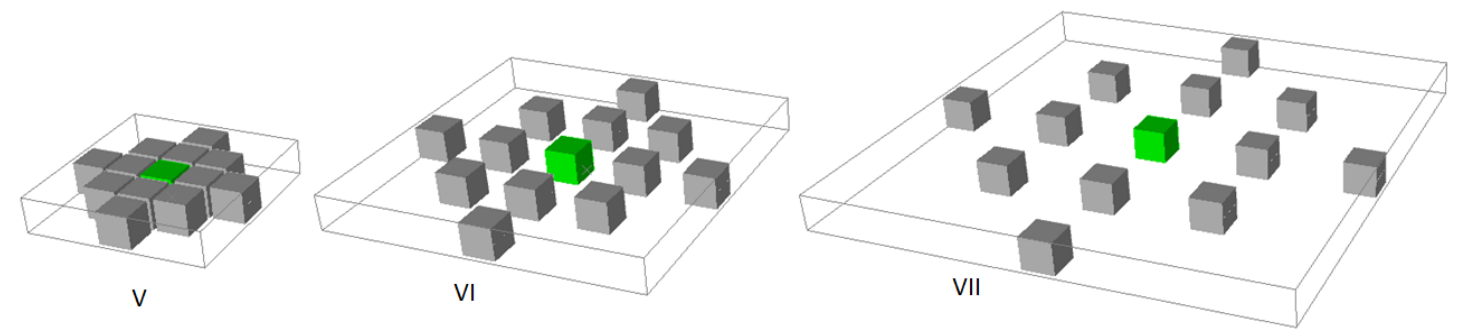

Figura 4.5: Estruturas de template de busca em 3D, configurações (V), (VI) e (VII), onde, captura-se informação cada vez mais abrangente. 
Teorema 4.1 Teorema de Bayes: assim como descrito, as variáveis na definição (4.1), pode-se escrever o seguinte:

$$
P\left\{S(u)=s_{k} \mid d_{n}=\tau_{n}\right\}=\frac{P\left\{d_{n}=\tau_{n} \mid S(u)=s_{k}\right\} P\left\{S(u)=s_{k}\right\}}{P\left\{d_{n}=\tau_{n}\right\}},
$$

onde

$$
P\left\{d_{n}=\tau_{n}\right\}=\sum_{k=1}^{K} P\left\{d_{n}=\tau_{n} \mid S(u)=s_{k}\right\} P\left\{S(u)=s_{k}\right\} .
$$

Segundo Strebelle (2002, p. 15), finitas realizações simuladas podem apresentar proporções globais significativamente diferentes da distribuição amostral. Conforme esse autor, isso ocorre particularmente se as covariâncias multi-pontos são herdadas a partir da imagem de treinamento com um histograma diferente da distribuição objetivo. Ou então pode ser o caso de um template de busca grosseiro, onde se recomenda utilizar outro template de busca de probabilidade que reconheça melhor as estruturas nas células estudadas e assim reproduzir probabilidades a posteriori ${ }^{2}$ das $K$ categorias mais próximos da probabilidade a priori $^{3}$ de $p_{k}$.

\subsection{Código da estatística multi-pontos}

A técnica da estatística multi-pontos (MPS) já foi implementada em vários softwares, tais como, ISATIS ${ }^{4}, \mathrm{SGeMS}^{5}$ e GSLIB ${ }^{6}$, nos quais os dois últimos softwares utilizaram o código Fortran 90 incluindo para uma implementação em 3D do algoritmo utilizando árvores de busca, sendo os mesmos escritos segundo notação e codificação GSLIB. Já o software ISATIS é um software comercial com código fonte não disponível.

As probabilidades condicionais são inferidas a partir da imagem de treinamento. O tamanho (número de pixels ou voxels) das imagens de treinamento e da imagem de simulação das células podem ser diferentes, mas suas categorias devem ser iguais. As unidades das coordenadas correspondem ao espaçamento dos nós da rede a ser simulada. Os vetores modelos são listados por módulo de incremento, usando a distância euclideana tradicional ou qualquer variograma baseado em distância. Ao usar uma célula, os modelos podem variar de uma célula para outra, mas um modelo de dados deve ser especificado por um célula de simulação.

Posteriormente, os parâmetros que serão usados para gerar as realizações simuladas para o código MPS em 2D e 3D serão mostrados.

\footnotetext{
${ }^{2}$ probabilidade a posteriori $p_{k}$ é mensurado após a simulação na imagem de saída

${ }^{3}$ probabilidade a priori $p_{k}$ é mensurado na imagem de treinamento

${ }^{4}$ Fonte: http://www.geovariances.com/IMG/pdf/_Geovariances_MPS-2.pdf

${ }^{5}$ Fonte: https://pangea.stanford.edu/groups/iamg09/workshop1.p $\mathrm{df}$

${ }^{6}$ Implementado por Strebelle (2000, p. 17)
} 


\section{Capítulo 5}

\section{Materiais e métodos}

Para testar a metodologia de simulação multi-ponto foram preparadas duas bases de dados contendo três litologias: uma base completa composta por 10.000 pontos dispostos em uma malha regular de 100 por 100 (Figura 5.1) e uma base reamostrada do arquivo completo composto por 400 pontos distribuídos em uma malha regular de 20 por 20 (Figura 5.2).

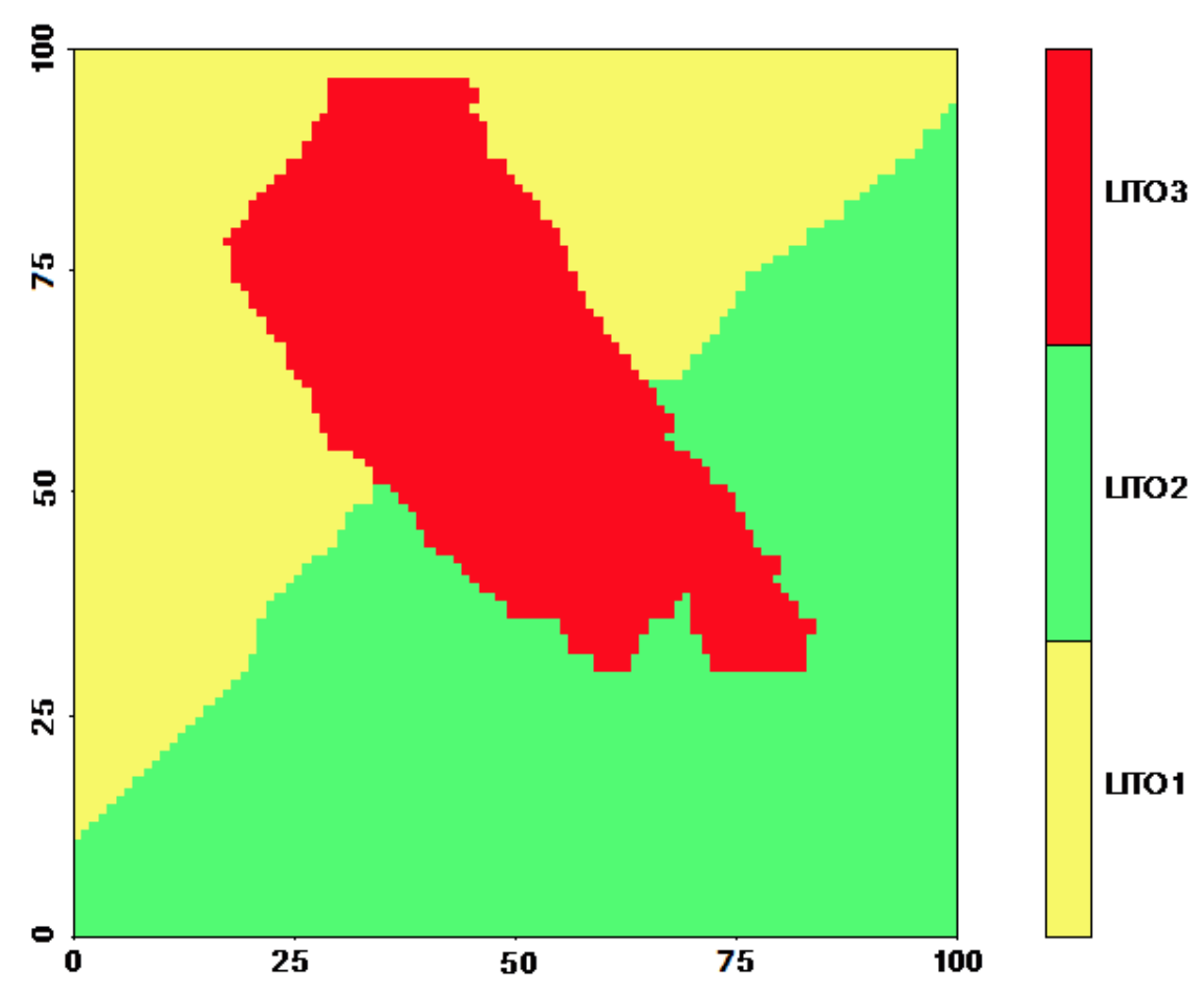

Figura 5.1: Base de dados completa constituída por 10.000 pontos.

$\mathrm{Na}$ realidade, a base de dados completa foi gerada para servir de imagem de treinamento para a simulação multi-ponto. A partir da base de dados reamostrada foi obtida uma amostra com 25 pontos de dados, que será usada para a simulação multi-ponto (Figura 5.3).

Assim, a imagem de treinamento (Figura 5.1) e a amostra de 25 pontos (Figura 5.3) 


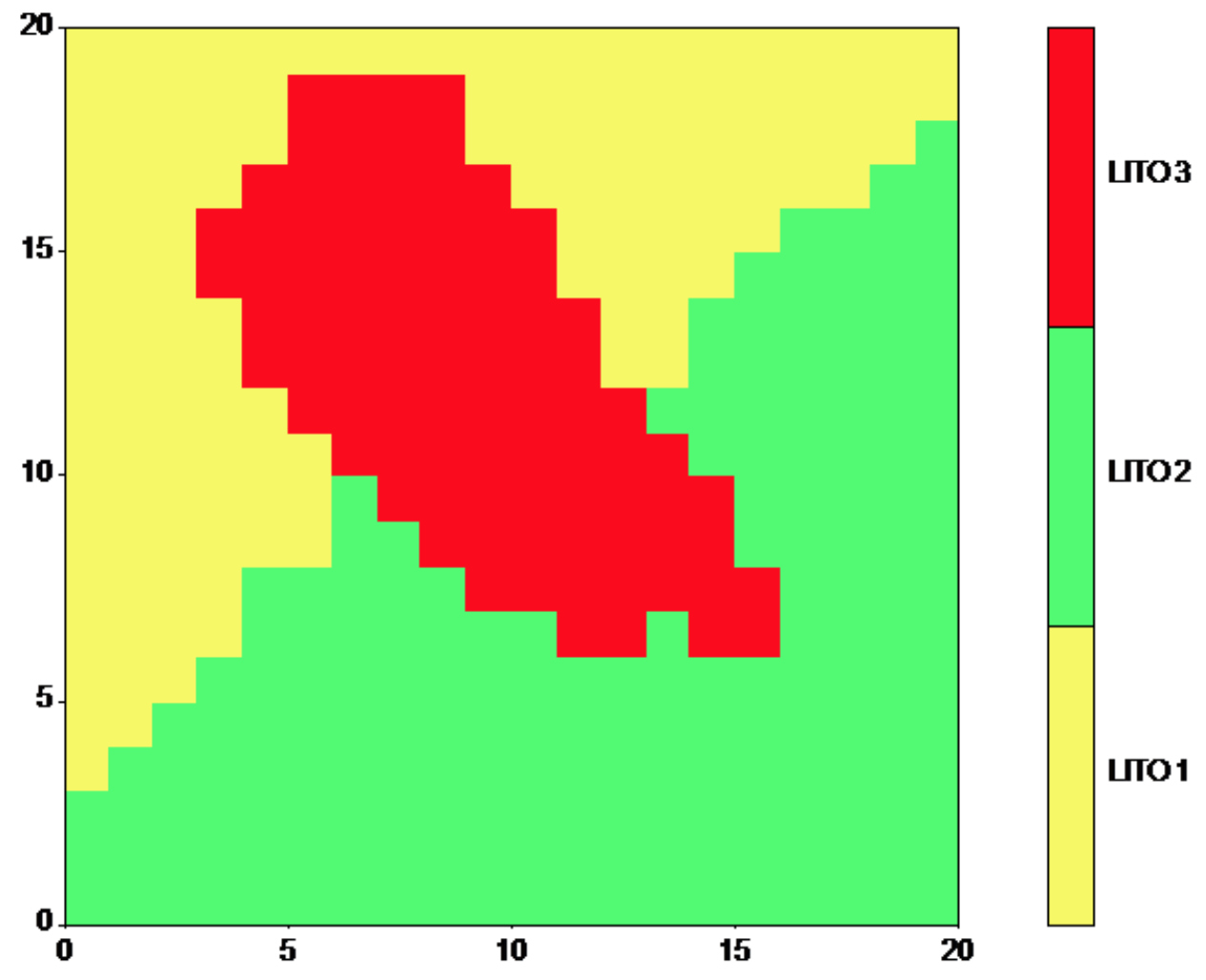

Figura 5.2: Base de dados reamostrada composta por 400 pontos.

constituem os materiais dessa pesquisa. Evidentemente, a amostra relativamente pequena com apenas 25 pontos de dados (0,25\% da Figura 5.1) foi considerada tanto para testar a metodologia multi-ponto, bem como para visualizar os resultados em uma escala razoável.

O algoritmo a ser usado foi descrito no capítulo 4. Tendo em vista a atualização do algoritmo proposto originalmente por Strebelle (2000, p. 6-13), as duas aproximações serão consideradas nesta tese, justamente para mostrar as diferenças causadas ao assumir probabilidades marginais para templates $\tau_{n}$ que nunca ocorrem, ou seja, com probabilidades nulas.

Além disso, como um dos objetivos é proporcionar o mapeamento da zona de incerteza para cada realização multi-ponto, os pixels simulados que apresentam variâncias maiores ou iguais a 0,20 foram destacadas e consideradas pertencentes à zona de incerteza, conforme proposta de Yamamoto et al. (2012, p. 149, expressão 3.38 neste trabalho). Para possibilitar a comparação das realizações multi-ponto com interpolações diretas das proporções, obtevese o mapa interpolado com a zona de incerteza mapeada (Figura 5.4). A zona de incerteza assim obtida servirá de referência à realização multi-ponto que deverá apresentar menor incerteza, haja vista estar baseada em probabilidades condicionais.

O programa que utilizou-se para realizar o desenvolvimento dos procedimentos da metodologia multi-ponto foi o software livre R (versão 3.0.0 RC), o código fonte do programa utilizado encontra-se no Apêndice 7.2. 


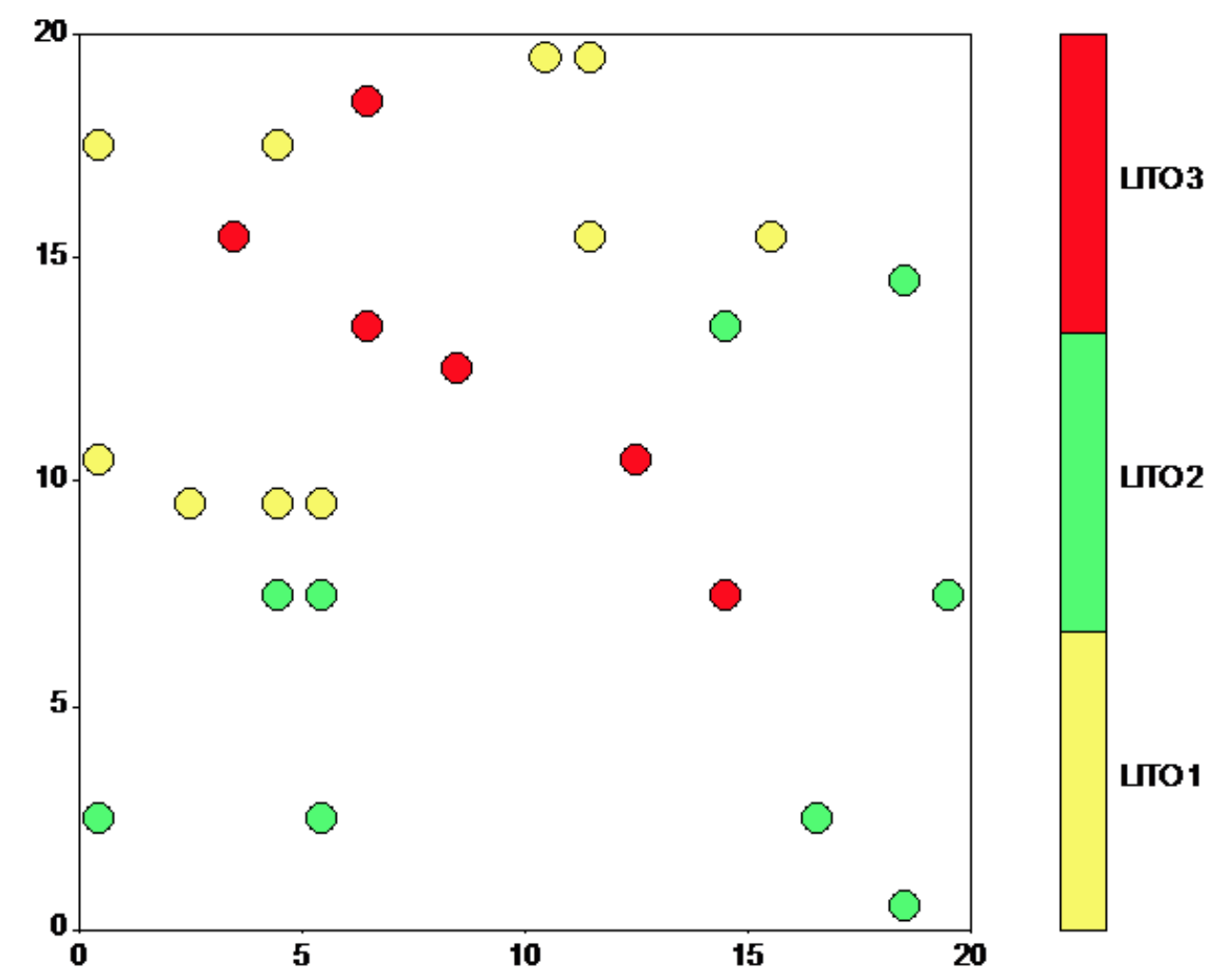

Figura 5.3: Mapa de localização dos pontos da amostra com 25 pontos.

\subsection{Mapeamento da árvore de probabilidades}

Na dinâmica de conseguir mapear as probabilidades de um evento acontecer através de um determinado template de busca $\tau_{n}$, utilizou-se um vetor dinâmico para o mapeamento das probabilidades, que segue as probabilidades mapeadas por meio da equação (3.42).

Nesta seção são apresentadas as três metodologias para o estudo da geoestatística multipontos.

\subsubsection{Metodologia por Yamamoto}

Esta metodologia é baseada no cálculo de uma única probabilidade estimada através da resolução do sistema de equações em (3.35) e os pesos $w_{i}$ estimados alocados para estimativa do valor $I_{M Q}^{*}\left(S\left(u_{0}\right) ; k\right)$ desde a equação (3.37), (Yamamoto et al., 2012).

\subsubsection{Metodologia por Strebelle}

Sabe-se muito pouco dos detalhes da implementação da metodologia desenvolvida por Strebelle (2000) dentro do software SGeMS e pelo GSLIB. Mas, pela metodologia descrita, tem-se, que utiliza uma estrutura de dados dinâmicos para o escaneamento das probabilidades condicionais desde uma imagem de treinamento dentro do espaço amostral fixado pelo template de busca, as quais são utilizadas na estimativa de cada célula não amostrada. 


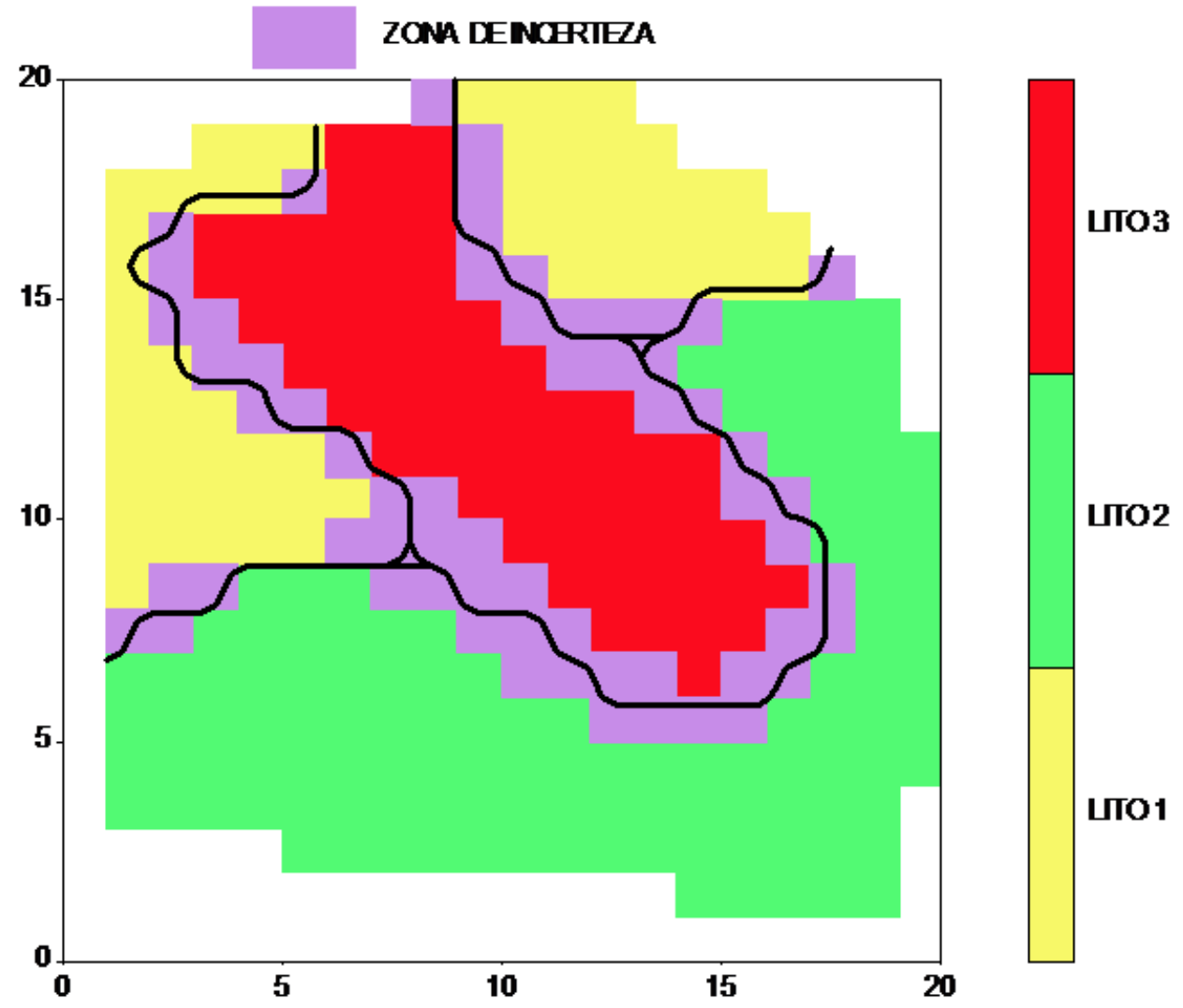

Figura 5.4: Tipos interpolados e mapeamento da zona de incerteza entre os tipos interpolados, conforme metodologia proposta por Yamamoto et al. (2012).

Tem-se dúvida em relação à terminologia utilizada sobre estrutura de dados dinâmicos, se este se refere ao template de busca ou à arvore de probabilidades condicionais. Desta forma, repassa-se as probabilidades condicionais para a imagem de estudo as quais são alocadas dentro de uma árvore imbricada para a expressão (3.42).

\subsubsection{Nova metodologia para o estudo multi-ponto}

Analisou-se as diversas informações contidas nos trabalhos de Strebelle (2000), Strebelle (2002) e Remy et al. (2009), desta forma separou-se a metodologia em três partes:

1. Escolha do template de busca e mapeia-se as probabilidades condicionais;

2. Mecanismo de utilização da árvore imbricada e dinâmica das probabilidades condicionais;

3. Simulação, alocação e atualização da imagem simulada para cada célula vazia.

\section{Parte 1}

Para esta etapa do algoritmo, primeiramente, escolhe-se o template de busca $\tau_{\alpha}$, assim como definido nesta tese, $n=12$, escolhou-se a configuração (V) da Figura (4.5), uma estrutura de template de busca em 3D, mas será trabalhada em 2D. 
Portanto, veja-se na Figura (4.1) as posições das $u_{\alpha}$ s, que estão representadas no espaço $3 \mathrm{D}$ e $u$ centralizado de cor verde, significando o sinal verde para alocar ocorrências de eventos e assim como de seus eventos vizinhos a ele. Desta forma, estas células (voxel) encontram-se espacialmente localizada sobre um arranjo de vetores $\left(u_{n}\right)$ com suas respectivas direções $(h)$, assim como descrito na Figura (4.2). Da mesma forma, pode-se descrever $u=(0,0,0)$ e seus vetores vizinhos definidos como:

$$
\begin{aligned}
& h_{1}=(0,1,0) \quad \rightarrow u_{1}=u+h_{1} \\
& h_{2}=(1,0,0) \quad \rightarrow u_{2}=u+h_{2} \\
& h_{3}=(0,-1,0) \quad \rightarrow u_{3}=u+h_{3} \\
& h_{4}=(-1,1,0) \quad \rightarrow u_{4}=u+h_{4} \\
& h_{5}=(1,1,0) \quad \rightarrow u_{5}=u+h_{5} \\
& h_{6}=(1,-1,0) \quad \rightarrow u_{6}=u+h_{6} \\
& h_{7}=(-1,-1,0) \rightarrow u_{7}=u+h_{7} \\
& h_{8}=(-1,1,0) \quad \rightarrow u_{8}=u+h_{8} \\
& h_{9}=(0,2,0) \quad \rightarrow u_{9}=u+h_{9} \\
& h_{10}=(0,-2,0) \quad \rightarrow u_{10}=u+h_{10} \\
& h_{11}=(2,1,0) \quad \rightarrow u_{11}=u+h_{11} \\
& h_{12}=(-2,1,0) \quad \rightarrow u_{12}=u+h_{12} \quad \text { (casso de estudo da tese) } \\
& h_{n}=(\ominus, \odot, \oslash) \quad \rightarrow u_{n}=u+h_{n}
\end{aligned}
$$

De maneira que pode-se escolher um número maior de vetores $h_{\alpha}$, tal que $\alpha$ seja grande, por exemplo $\alpha=100$, segundo um template $\tau_{n}$ que se queira aplicar aos dados.

Assim, captura-se a cada nó os valores de seus vizinhos numa matriz, assim como descrito 
na Figura (4.3), veja-se:

$$
\begin{aligned}
S(u) & =s_{0} \\
S\left(u_{1}\right) & =s_{1} \\
S\left(u_{2}\right) & =s_{2} \\
S\left(u_{3}\right) & =s_{3} \\
S\left(u_{4}\right) & =s_{4} \\
S\left(u_{5}\right) & =s_{5} \\
S\left(u_{6}\right) & =s_{6} \\
S\left(u_{7}\right) & =s_{7} \\
S\left(u_{8}\right) & =s_{8} \\
S\left(u_{9}\right) & =s_{9} \\
S\left(u_{10}\right) & =s_{10} \\
S\left(u_{11}\right) & =s_{11} \\
S\left(u_{12}\right) & =s_{12} \quad \text { (casso de estudo da tese) } \\
& \vdots
\end{aligned}
$$

Desta forma, aloca-se numa matriz (Imagem-treinamento $\left.=X^{1}\right) t \times(n+1)$ os valores avaliados de $S\left(u_{\alpha}\right)$, onde $\alpha=1, \ldots, n$ para cada i-ésimo nó das células na imagem de treinamento, assim como: $[i]=,\left[s_{0}^{i}, s_{1}^{i}, s_{2}^{i}, s_{3}^{i}, s_{4}^{i}, s_{5}^{i}, s_{6}^{i}, s_{7}^{i}, s_{8}^{i}, s_{9}^{i}, s_{10}^{i}, s_{11}^{i}, s_{12}^{i}\right]$ para cada $i=1$ até o último nó da imagem de treinamento, onde cada $s$ pode tomar os $K$ valores da variável categórica, totalizando $K^{t}$, onde $t$ o tamanho de nós nas células em estudo. No caso em estudo $i=1,2, \ldots, 10.000$. O resultado da construção dessa matriz será a matriz que carregará as ocorrências de eventos na imagem de treinamento. Utilizando a função table e prop.table do $\mathrm{R}$, tabula-se todas as possíveis combinações entre as colunas da matriz $X$.

$$
X=\left[\begin{array}{ccccccccccccccc}
s_{0}^{1} & s_{1}^{1} & s_{2}^{1} & s_{3}^{1} & s_{4}^{1} & s_{5}^{1} & s_{6}^{1} & s_{7}^{1} & s_{8}^{1} & s_{9}^{1} & s_{10}^{1} & s_{11}^{1} & s_{12}^{1} & \ldots & s_{n}^{1} \\
s_{0}^{2} & s_{1}^{2} & s_{2}^{2} & s_{3}^{2} & s_{4}^{2} & s_{5}^{2} & s_{6}^{2} & s_{7}^{2} & s_{8}^{2} & s_{9}^{2} & s_{10}^{2} & s_{11}^{2} & s_{12}^{2} & \ldots & s_{n}^{2} \\
s_{0}^{3} & s_{1}^{3} & s_{2}^{3} & s_{3}^{3} & s_{4}^{3} & s_{5}^{3} & s_{6}^{3} & s_{7}^{3} & s_{8}^{3} & s_{9}^{3} & s_{10}^{3} & s_{11}^{3} & s_{12}^{3} & \ldots & s_{n}^{3} \\
\vdots & \vdots & \vdots & \vdots & \vdots & \vdots & \vdots & \vdots & \vdots & \vdots & \vdots & \vdots & \vdots & \ddots & \vdots \\
s_{0}^{t} & s_{1}^{t} & s_{2}^{t} & s_{3}^{t} & s_{4}^{t} & s_{5}^{t} & s_{6}^{t} & s_{7}^{t} & s_{8}^{t} & s_{9}^{t} & s_{10}^{t} & s_{11}^{t} & s_{12}^{t} & \ldots & s_{n}^{t}
\end{array}\right]
$$

Para o caso em estudo da tese $t=10.000$.

\footnotetext{
${ }^{1}$ Imagem - treinamento $=X$ : é nome da matriz no código fonte
} 


\section{Parte 2}

Em contraste, a árvore de probabilidades condicionais descrita por Strebelle (2000), onde utiliza o nível zero de probabilidade, ou seja $p_{k}, k=1, \ldots, K$, esses eventos não pertencem ao subconjunto de eventos do espaço amostral condicionado. Portanto, uma alternativa ainda melhor é proposta da seguinte maneira: primeiramente, as probabilidades do nível zero são expurgadas e constrói-se a seguinte estrutura de abordagem imbricada por níveis:

1. Se o nó escolhido para simulação pertence ao nível $n$ de probabilidades condicionais dado o $\tau_{n}$, procura-se a probabilidade associada através da expressão (3.42) e associa-se a matriz $X$ de forma que se obtenha as probabilidades condicionais:

$$
\operatorname{Prob}\left\{\frac{S(u)=s_{k}, S\left(u_{1}\right), S\left(u_{2}\right), S\left(u_{3}\right), \ldots, S\left(u_{n}\right)}{S\left(u_{1}\right), S\left(u_{2}\right), S\left(u_{3}\right), \ldots, S\left(u_{n}\right)}\right\}, \forall k=1, \ldots, K
$$

$$
\frac{\text { Nro. de eventos }\left\{S(u)=s_{k}, S\left(u_{1}\right), S\left(u_{2}\right), S\left(u_{3}\right), \ldots, S\left(u_{n}\right)\right\}}{\text { Nro. de eventos }\left\{S\left(u_{1}\right), S\left(u_{2}\right), S\left(u_{3}\right), \ldots, S\left(u_{n}\right)\right\}}, \forall k=1, \ldots, K,
$$

e por 3.50 tem-se: $\operatorname{Prob}\left\{S(u)=s_{k} \mid d_{n}\right\} \simeq \frac{c_{k}}{c}$, onde, $\sum_{k=1}^{K} c_{k}=c$.

Caso o evento descrito no denominador não exista, diminui-se um nível, retirando o último vetor $S\left(u_{n}\right)$ que ocasionou o nível $d_{n}$ e repete-se até encontrar um subconjunto de estatísticas suficientes para formar uma probabilidade condicional válida.

2. Se o nó escolhido para simulação pertence ao nível $n^{\prime}=n-1$ de probabilidades condicionais dado o $\tau_{n^{\prime}}$, procura-se a probabilidade associada através da expressão (3.42) e associa-se a matriz $X$ de forma que se obtenha as probabilidades condicionais:

$$
\operatorname{Prob}\left\{\frac{S(u)=s_{k}, S\left(u_{1}\right), S\left(u_{2}\right), S\left(u_{3}\right), \ldots, S\left(u_{n^{\prime}}\right)}{S\left(u_{1}\right), S\left(u_{2}\right), S\left(u_{3}\right), \ldots, S\left(u_{n^{\prime}}\right)}\right\}, \forall k=1, \ldots, K
$$

$$
\frac{\text { Nro. de eventos }\left\{S(u)=s_{k}, S\left(u_{1}\right), S\left(u_{2}\right), S\left(u_{3}\right), \ldots, S\left(u_{n^{\prime}}\right)\right\}}{\text { Nro. de eventos }\left\{S\left(u_{1}\right), S\left(u_{2}\right), S\left(u_{3}\right), \ldots, S\left(u_{n^{\prime}}\right)\right\}}, \forall k=1, \ldots, K,
$$

e por 3.50 tem-se: $\operatorname{Prob}\left\{S(u)=s_{k} \mid d_{n^{\prime}}\right\} \simeq \frac{c_{k}}{c}$, onde, $\sum_{k=1}^{K} c_{k}=c$.

Caso o conjunto de eventos no denominador não exista, diminui-se um nível, retirando o último vetor $S\left(u_{n^{\prime}}\right)$, que ocasionou o nível $d_{n^{\prime}}$ e repete-se até encontrar um subconjunto de estatísticas suficientes para formar uma probabilidade condicional válida. Da mesma forma, tem-se outras $\left(\begin{array}{c}n \\ n-1\end{array}\right)$ combinações, exemplo: $S(u)=s_{k}, S\left(u_{2}\right), S\left(u_{3}\right), \ldots, S\left(u_{n}\right)$.

3. Se o nó escolhido para simulação pertence ao nível $n^{\prime \prime}=n-2$ de probabilidades condicionais dado o $\tau_{n^{\prime \prime}}$, procura-se a probabilidade associada através da expressão (3.42) e associa-se a matriz $X$ de forma que se obtenha as probabilidades condicionais: 


$$
\operatorname{Prob}\left\{\frac{S(u)=s_{k}, S\left(u_{1}\right), S\left(u_{2}\right), S\left(u_{3}\right), \ldots, S\left(u_{n^{\prime \prime}}\right)}{S\left(u_{1}\right), S\left(u_{2}\right), S\left(u_{3}\right), \ldots, S\left(u_{n^{\prime \prime}}\right)}\right\}, \forall k=1, \ldots, K
$$

$\frac{\text { Nro. de eventos }\left\{S(u)=s_{k}, S\left(u_{1}\right), S\left(u_{2}\right), S\left(u_{3}\right), \ldots, S\left(u_{n^{\prime \prime}}\right)\right\}}{\text { Nro. de eventos }\left\{S\left(u_{1}\right), S\left(u_{2}\right), S\left(u_{3}\right), \ldots, S\left(u_{n^{\prime \prime}}\right)\right\}}, \forall k=1, \ldots, K$,

e por 3.50 tem-se: $\operatorname{Prob}\left\{S(u)=s_{k} \mid d_{n^{\prime \prime}}\right\} \simeq \frac{c_{k}}{c}$, onde, $\sum_{k=1}^{K} c_{k}=c$.

Caso o conjunto de eventos no denominador não exista, diminui-se um nível, retirando o último vetor $S\left(u_{n^{\prime \prime}}\right)$, que ocasionou o nível $d_{n^{\prime \prime}}$ e repete-se até encontrar um subconjunto de estatísticas suficientes para formar uma probabilidade condicional válida. Da mesma forma, tem-se outras $\left(\begin{array}{c}n \\ n-2\end{array}\right)$ combinações, exemplo: $S\left(u_{3}\right), S\left(u_{4}\right), \ldots, S\left(u_{n-1}\right), S\left(u_{n}\right)$.

4. Desta forma, realiza-se sucessivamente para construir as probabilidades condicionais de nós menores, assim como descrito a seguir.

5. Se o nó escolhido para simulação pertence ao nível $n^{\prime \prime \prime}=3$ de probabilidades condicionais dado o $\tau_{n^{\prime \prime \prime}}$, procura-se a probabilidade associada através da expressão (3.42) e associa-se a matriz $X$ de forma que se obtenha as probabilidades condicionais:

$$
\operatorname{Prob}\left\{\frac{S(u)=s_{k}, S\left(u_{1}\right), S\left(u_{2}\right), S\left(u_{3}\right)}{S\left(u_{1}\right), S\left(u_{2}\right), S\left(u_{3}\right)}\right\}, \forall k=1, \ldots, K
$$

$$
\frac{\text { Nro. de eventos }\left\{S(u)=s_{k}, S\left(u_{1}\right), S\left(u_{2}\right), S\left(u_{3}\right)\right\}}{\text { Nro. de eventos }\left\{S\left(u_{1}\right), S\left(u_{2}\right), S\left(u_{3}\right)\right\}}, \forall k=1, \ldots, K,
$$

e por 3.50 tem-se: $\operatorname{Prob}\left\{S(u)=s_{k} \mid d_{n^{\prime \prime \prime}}\right\} \simeq \frac{c_{k}}{c}$, onde, $\sum_{k=1}^{K} c_{k}=c$.

Caso o conjunto de eventos no denominador não exista, diminui-se um nível, retirando o último vetor $S\left(u_{n^{\prime \prime \prime}}\right)$, que ocasionou o nível $d_{n^{\prime \prime \prime}}$ e repete-se até encontrar um subconjunto de estatísticas suficientes para formar uma probabilidade condicional valida. Da mesma forma, tem-se outras $\left(\begin{array}{l}n \\ 3\end{array}\right)$ combinações, exemplo: $S\left(u_{7}\right), S\left(u_{9}\right), S\left(u_{10}\right)$.

6. Se o nó escolhido para simulação pertence ao nível $n^{\prime \prime \prime \prime}=2$ de probabilidades condicionais dado o $\tau_{n^{\prime \prime \prime \prime}}$, procura-se a probabilidade associada através da expressão (3.42) e associa-se a matriz $X$ de forma que se obtenha as probabilidades condicionais:

$$
\operatorname{Prob}\left\{\frac{S(u)=s_{k}, S\left(u_{1}\right), S\left(u_{2}\right)}{S\left(u_{1}\right), S\left(u_{2}\right)}\right\}, \forall k=1, \ldots, K
$$

$$
\frac{\text { Nro. de eventos }\left\{S(u)=s_{k}, S\left(u_{1}\right), S\left(u_{2}\right), S\left(u_{3}\right)\right\}}{\text { Nro. de eventos }\left\{S\left(u_{1}\right), S\left(u_{2}\right), S\left(u_{3}\right)\right\}}, \forall k=1, \ldots, K,
$$

e por 3.50 tem-se: $\operatorname{Prob}\left\{S(u)=s_{k} \mid d_{n^{\prime \prime \prime \prime}}\right\} \simeq \frac{c_{k}}{c}$, onde, $\sum_{k=1}^{K} c_{k}=c$. 
Caso o conjunto de eventos no denominador não exista, diminui-se um nível, retirando o último vetor $S\left(u_{n^{\prime \prime \prime \prime}}\right)$, que ocasionou o nível $d_{n^{\prime \prime \prime \prime}}$ e repete-se até encontrar um subconjunto de estatísticas suficientes para formar uma probabilidade condicional valida. Da mesma forma tem-se outras $\left(\begin{array}{l}n \\ 2\end{array}\right)$ combinações, exemplo: $S\left(u_{7}\right), S\left(u_{12}\right)$.

7. Se o nó escolhido para simulação pertence ao nível $n^{\prime \prime \prime \prime \prime}=1$ de probabilidades condicionais dado o $\tau_{n^{\prime \prime \prime \prime \prime \prime}}$, procura-se a probabilidade associada através da expressão (3.42) e associa-se a matriz $X$ de forma que se obtenha as probabilidades condicionais:

$$
\begin{gathered}
\operatorname{Prob}\left\{\frac{S(u)=s_{k}, S\left(u_{1}\right)}{S\left(u_{1}\right)}\right\}, \forall k=1, \ldots, K, \\
\frac{\text { Nro. de eventos }\left\{S(u)=s_{k}, S\left(u_{1}\right)\right\}}{\text { Nro. de eventos }\left\{S\left(u_{1}\right)\right\}}, \quad \forall k=1, \ldots, K,
\end{gathered}
$$

e por 3.50 tem-se: $\operatorname{Prob}\left\{S(u)=s_{k} \mid d_{n^{\prime \prime \prime \prime \prime}}\right\} \simeq \frac{c_{k}}{c}$, onde, $\sum_{k=1}^{K} c_{k}=c$.

Caso o conjunto de eventos no denominador não exista ou não possua vizinhos dentro do template de busca, este nó terá que aguardar o novo passeio aleatório, até pelo menos obter um nó vizinho existente. Em contraste, a metodologia de Strebelle (2000) atribui probabilidade a priori $p_{k} \forall k=1, \ldots, K$ neste caso.

Pouco se sabe sobre o dinamismo reverso de níveis de probabilidades que é realizado no SNESIM, e que neste trabalho foi desenvolvido (ver código fonte em 7.2).

\section{Parte 3}

Em cada nó, onde encontrou-se eventos válidos dentro do espaço amostral condicional, simula-se um valor aleatório respeitando suas devidas probabilidades encontradas.

Desta forma, uma realização de uma imagem estocástica será gerada e assim repetir estes passos desde o passo 2 para obter uma nova realização, veja-se na seção seguinte várias realizações desde uma amostra de dados.

\subsection{Fluxograma multi-pontos}

A seguir mostre-se a figura (5.5), o passo a passo do algoritmo utilizando a nova metodologia proposta nesta tese através de um fluxograma. 


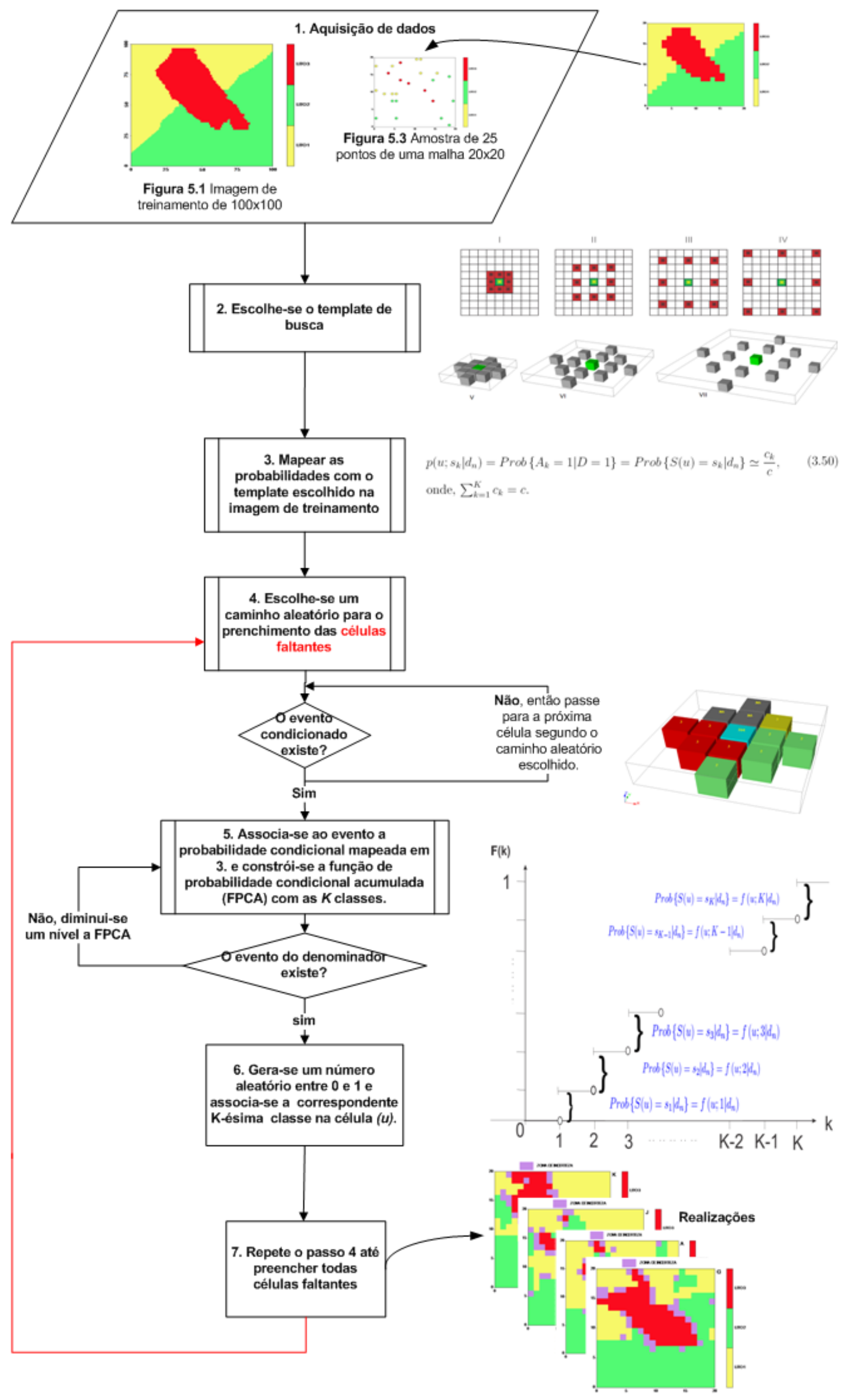

Figura 5.5: Fluxograma com a nova metodologia multi-pontos. 


\section{Capítulo 6}

\section{Resultados e discussão}

Os resultados obtidos da simulação multi-ponto conforme os métodos descritos no capítulo anterior encontram-se nas Figuras 6.1 6.2, 6.3 e 6.4, conforme Strebelle (2000) e de acordo com a metodologia proposta nesta tese usando o software R.

Como se pode observar nessas figuras, foram obtidas 12 realizações para ambas as metodologias com o objetivo de analisar a ergodicidade das mesmas. Além disso, todas as realizações estão com as zonas de incerteza destacadas.

As zonas de incerteza foram mapeadas aplicando-se a metodologia proposta por Yamamoto et al. (2012), que sugerem separar um evento certo do incerto, com base na sua variância. Como se trabalha com as probabilidades, ou seja, com proporções de tipos da variável categórica $\left(P_{k}\right)$, as variâncias associadas são facilmente determinadas como $P_{k}(1-$ $\left.P_{k}\right)$.

É importante ressaltar que em geoestatística é comum usar as realizações da simulação estocástica para determinar a variância ou incerteza associada à média das realizações. Mas, deve-se ter em mente que cada realização também está sujeita a uma incerteza, pois é condicional aos pontos amostrais. Na metodologia de Strebelle (2000) e na proposta da nova metodologia, consideram-se o mapeamento da zona de incerteza de cada realização multi-ponto como algo inovador e efetiva contribuição dessa pesquisa.

Comparando-se os resultados, verifica-se que a metodologia proposta por Strebelle (2000), proporciona realizações com incertezas maiores devido à atribuição de probabilidades marginais a templates que nunca ocorrem. Como se trata de método sequencial, ao assumir uma probabilidade à priori para simular um nó, esse método atualiza a base de dados e assim propaga-se para toda a região de estudo. A maior incerteza decorre do peso de probabilidades marginais ao invés das probabilidades condicionais.

Ao revermos esse fato, o algoritmo de Strebelle (2000) foi estudado à exaustão, que permitiu descobrir o peso de probabilidades marginais para templates que nunca ocorrem e, portanto, com probabilidades nulas. Assim, o algoritmo proposto nesse trabalho retorna ao nível imediatamente anterior àquele que foi verificado um template que nunca ocorre. Portanto, conforme a alteração proposta trabalha-se somente com probabilidades condicionais, 
Tabela 6.1: Frequências das litologias na imagem reamostrada de 400 pontos.

\begin{tabular}{|c|c|c|c|}
\hline \multicolumn{4}{|c|}{ Frequências } \\
\hline Litologia 1 & Litologia 2 & Litologia 3 & Total \\
\hline 127 & 185 & 88 & 400 \\
\hline
\end{tabular}

Tabela 6.2: Frequências das litologias conforme as realizações multi-ponto de acordo com a metodologia de Strebelle (2000).

\begin{tabular}{|c|c|c|c|c|}
\hline \multirow{2}{*}{ Realização } & \multicolumn{4}{|c|}{ Frequências } \\
\cline { 2 - 5 } & Litologia 1 & Litologia 2 & Litologia 3 & ZI \\
\hline 1 & 73 & 120 & 89 & 118 \\
\hline 2 & 75 & 153 & 76 & 96 \\
\hline 3 & 97 & 126 & 73 & 104 \\
\hline 4 & 72 & 135 & 100 & 93 \\
\hline 5 & 73 & 162 & 87 & 78 \\
\hline 6 & 67 & 170 & 84 & 79 \\
\hline 7 & 100 & 107 & 65 & 128 \\
\hline 8 & 72 & 156 & 71 & 101 \\
\hline 9 & 71 & 153 & 76 & 100 \\
\hline 10 & 82 & 152 & 82 & 84 \\
\hline 11 & 73 & 120 & 89 & 118 \\
\hline 12 & 75 & 153 & 76 & 96 \\
\hline Média: & 77,50 & 142,25 & 80,67 & 99,58 \\
\hline Desvio Padrão: & 9,97 & 19,02 & 9,26 & 15,02 \\
\hline
\end{tabular}

que são sempre maiores que as probabilidades marginais e, assim, com menores incertezas associadas.

Os resultados obtidos nas realizações devem ser comparados com a Figura 5.2, a imagem reamostrada da imagem de treinamento (Figura 5.1), pois a malha é idêntica às malhas das realizações efetuadas.

Assim, como ponto de partida mostra-se na Tabela 6.1 as frequências da imagem reamostrada em termos das três litologias que compõem uma variável categórica.

As estatísticas das realizações conforme a metodologia de Strebelle (2000) encontram-se na Tabela 6.2.

A síntese dos resultados das realizações conforme a alteração proposta neste trabalho encontra-se na Tabela 6.3 .

Como se pode observar nas Tabelas (6.2 e 6.3), os resultados das realizações multi-ponto de acordo com a alteração proposta desta tese são superiores (em relação à média) àqueles conforme a metodologia clássica de Strebelle (2000).

Embora os resultados observados na Tabela (6.4) pela metodologia Yamamoto et al. tenha problemas de estimativa nas bordas e seja gerada por uma única realização (única probabilidade), por se tratar de uma metodologia originada pela krigagem indicadora, ela ainda deve ser utilizada em casos que não se possua uma imagem de treinamento (informação a priori). 
Tabela 6.3: Frequências das litologias conforme as realizações multi-ponto de acordo com a nova metodologia proposta nesta tese.

\begin{tabular}{|c|c|c|c|c|}
\hline \multirow{2}{*}{ Realização } & \multicolumn{4}{|c|}{ Frequências } \\
\cline { 2 - 5 } & Litologia 1 & Litologia 2 & Litologia 3 & ZI \\
\hline 1 & 119 & 136 & 107 & 38 \\
\hline 2 & 97 & 174 & 89 & 40 \\
\hline 3 & 124 & 178 & 59 & 39 \\
\hline 4 & 107 & 141 & 116 & 36 \\
\hline 5 & 102 & 174 & 86 & 38 \\
\hline 6 & 109 & 169 & 82 & 40 \\
\hline 7 & 91 & 191 & 85 & 33 \\
\hline 8 & 97 & 146 & 103 & 54 \\
\hline 9 & 95 & 183 & 80 & 42 \\
\hline 10 & 107 & 166 & 82 & 45 \\
\hline 11 & 90 & 199 & 77 & 34 \\
\hline 12 & 147 & 156 & 59 & 38 \\
\hline Média: & 107,08 & 167,75 & 85,42 & 39,75 \\
\hline Desvio Padrão: & 15,68 & 18,87 & 16,44 & 5,31 \\
\hline
\end{tabular}

Tabela 6.4: Frequências das litologias conforme a realização multi-ponto de acordo com a metodologia Yamamoto et al..

\begin{tabular}{|c|c|c|c|c|c|}
\hline \multirow{2}{*}{ Realização } & \multicolumn{5}{|c|}{ Frequências } \\
\cline { 2 - 6 } & Litologia 1 & Litologia 2 & Litologia 3 & ZI & Nó não estimado \\
\hline 1 & 59 & 119 & 68 & 61 & 93 \\
\hline Média: & 59 & 119 & 68 & 61 & 93 \\
\hline
\end{tabular}

Neste sentido, as proporções simuladas conforme a nova aproximação (Tabela 6.3) são mais próximas àquelas iniciais (Tabela 6.1), mesmo considerando a zona de incerteza. As zonas de incerteza associadas às realizações multi-ponto conforme a alteração proposta são muito menores que aquelas mapeadas segundo as outras duas metodologias estudadas. 


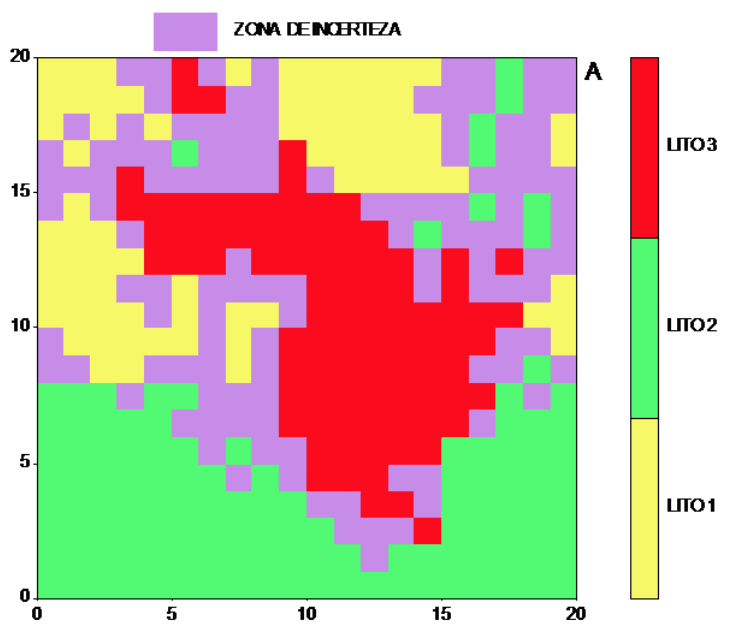

(a)

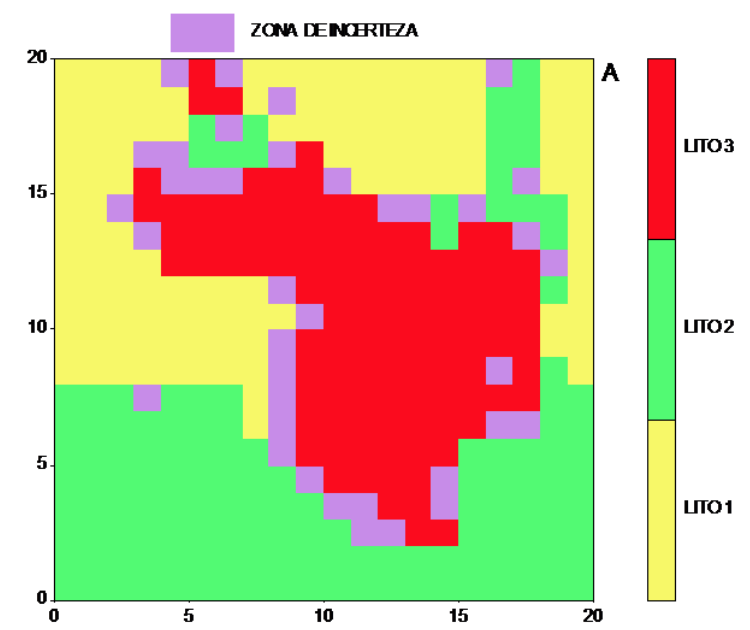

(b)

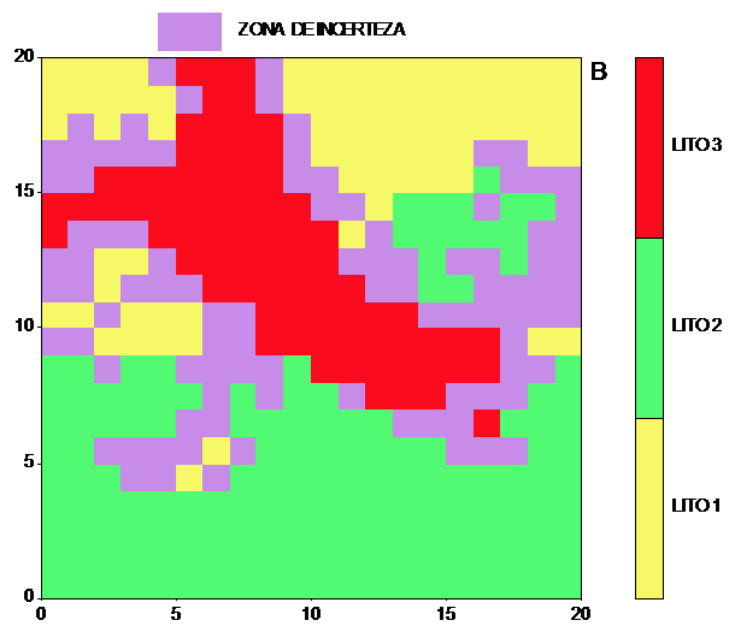

(c)

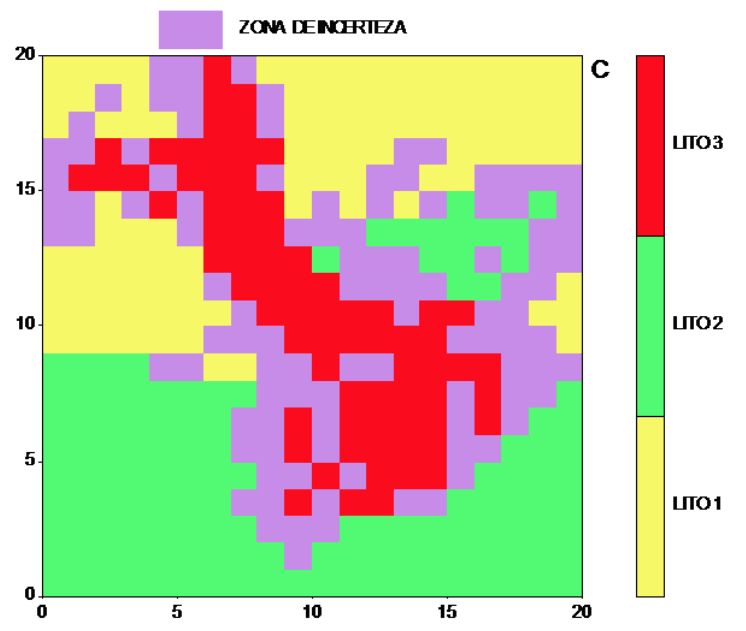

(e)

Rodriguez com R
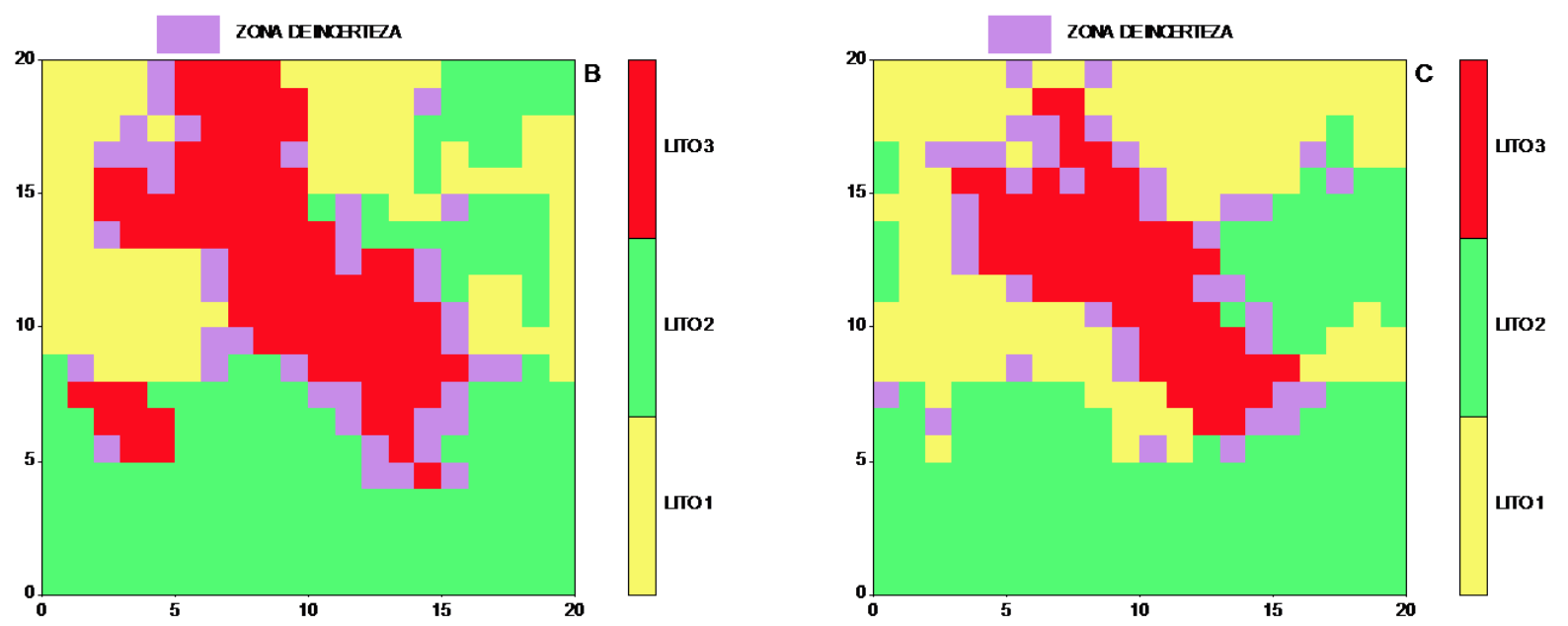

(d)

Figura 6.1: (a) $1^{\circ}$ realização segundo Strebelle, (b) $1^{\circ}$ realização segundo Rodriguez, (c) $2^{\circ}$ realização segundo Strebelle, (d) $2^{\circ}$ realização segundo Rodriguez, (e) $3^{\circ}$ realização segundo Strebelle, (f) $3^{\circ}$ realização segundo Rodriguez. 


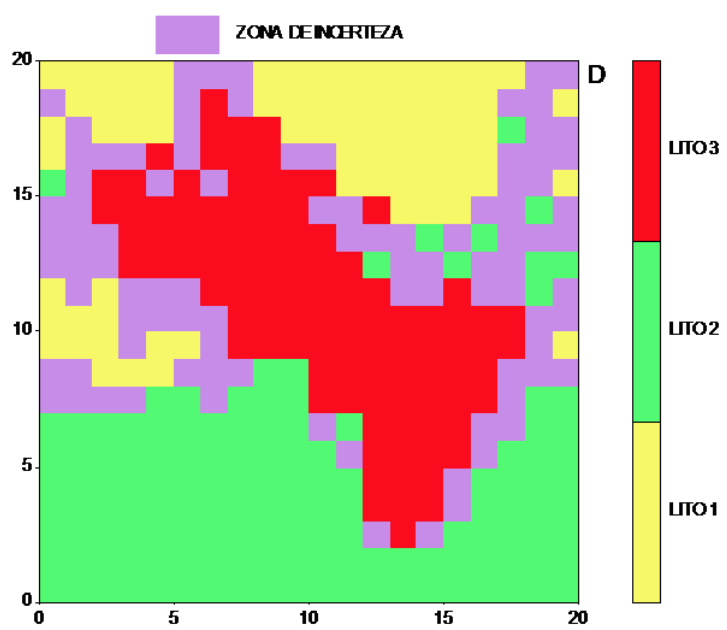

(g)

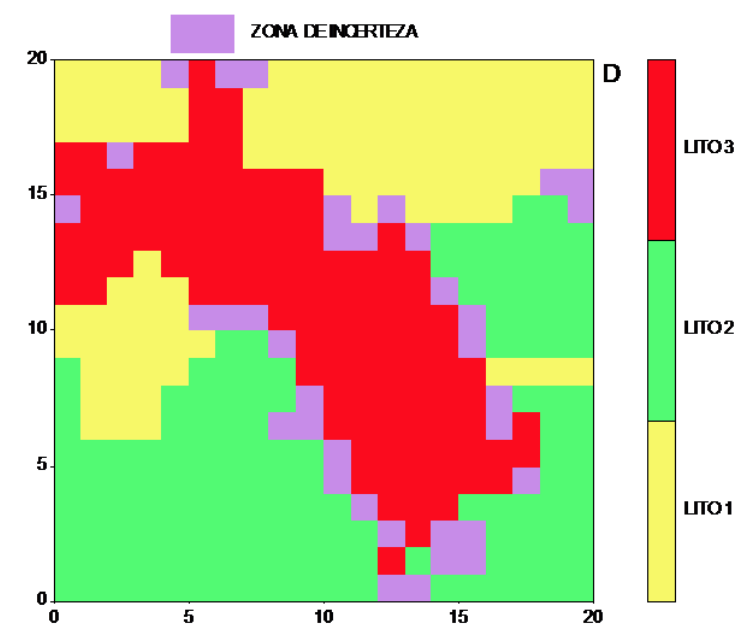

(h)

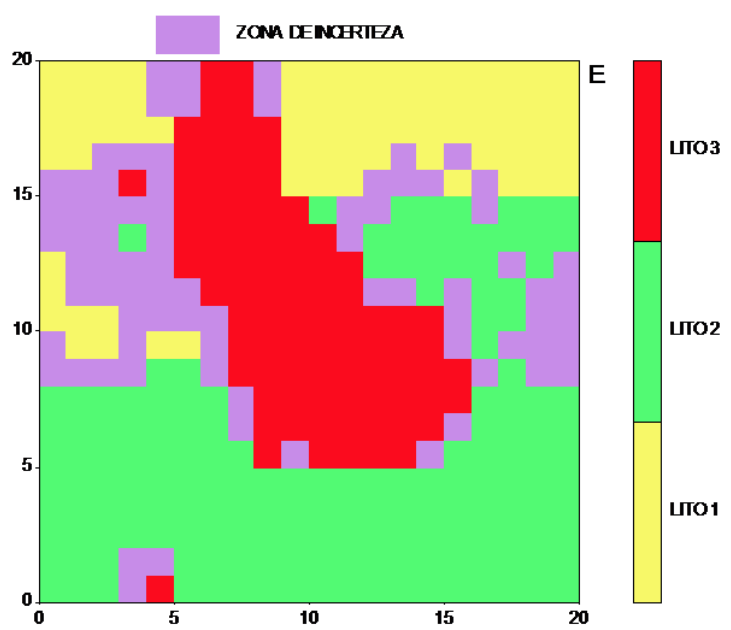

(i)

Rodriguez com R

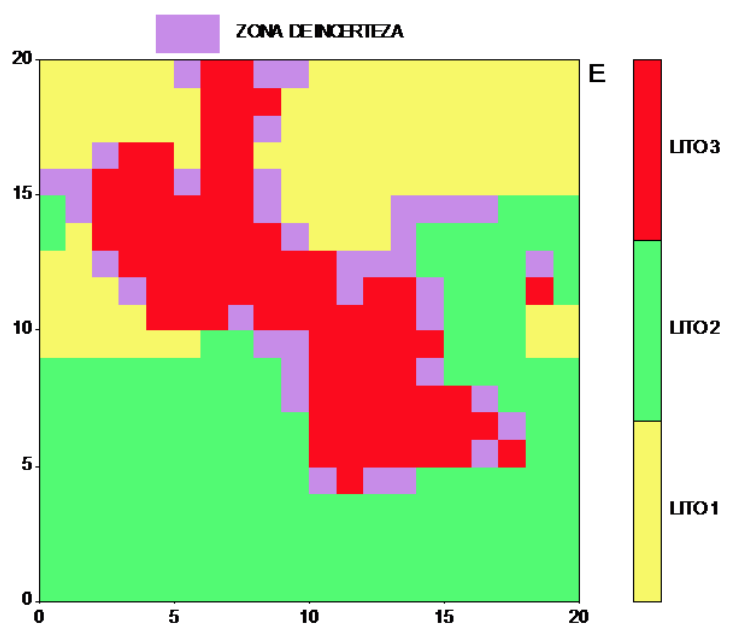

(j)

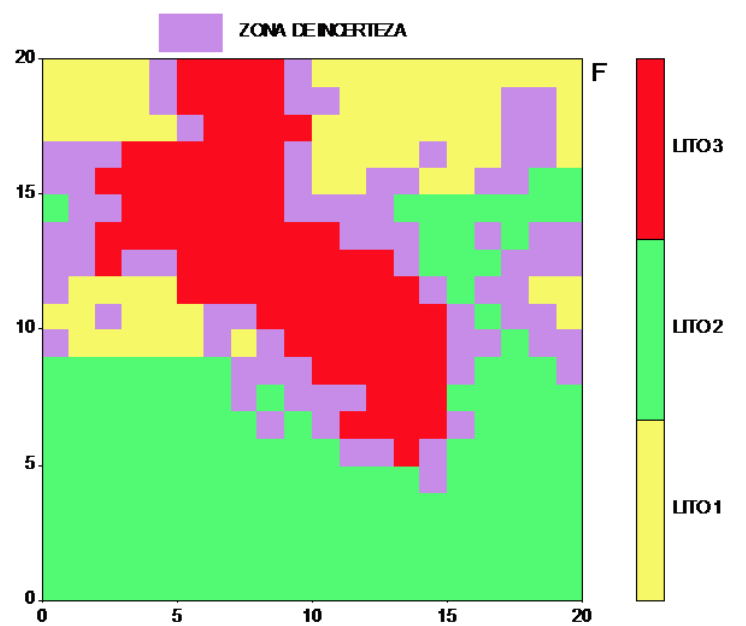

(k)

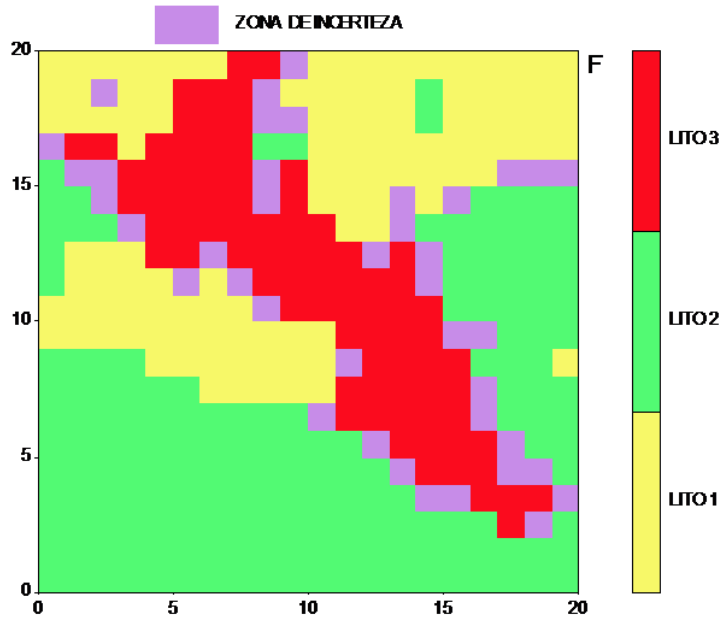

(1)

Figura 6.2: (g) $4^{\circ}$ realização segundo Strebelle, (h) $4^{\circ}$ realização segundo Rodriguez, (i) $5^{\circ}$ realização segundo Strebelle, (j) $5^{\circ}$ realização segundo Rodriguez, (k) $6^{\circ}$ realização segundo Strebelle, (l) $6^{\circ}$ realização segundo Rodriguez. 


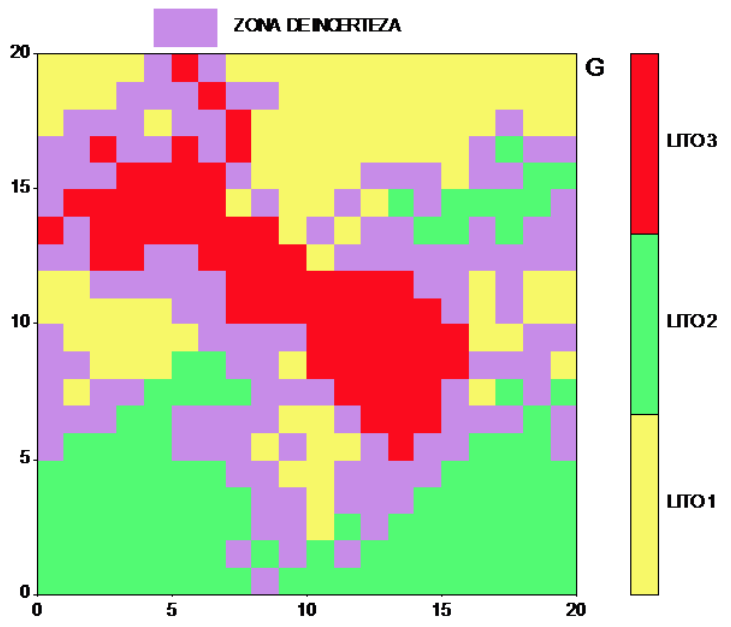

(m)

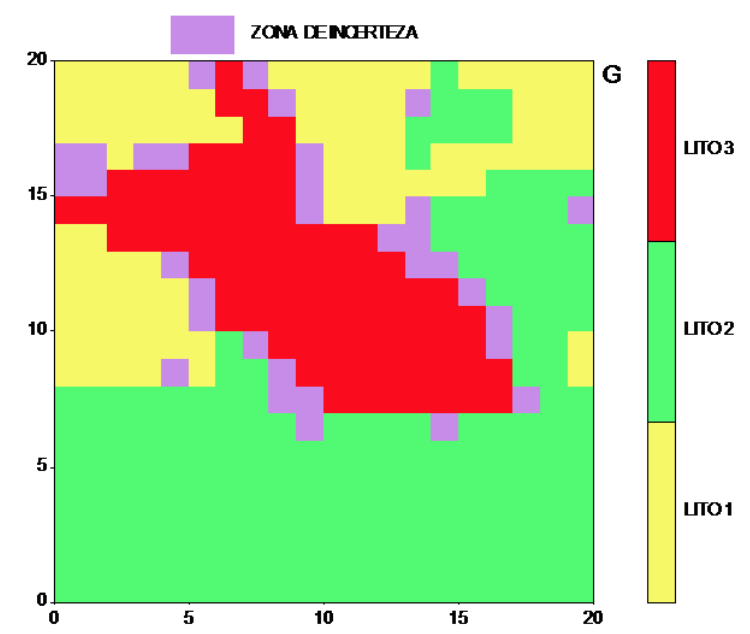

(n)

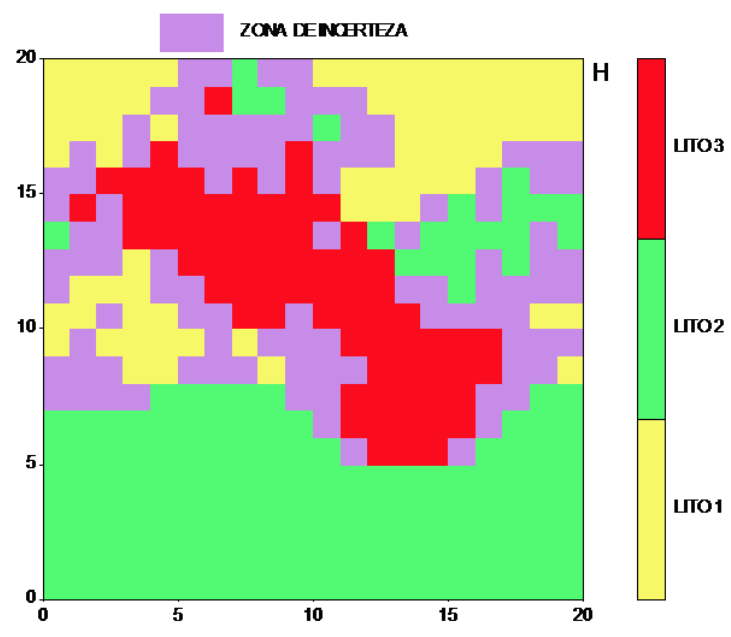

(o)

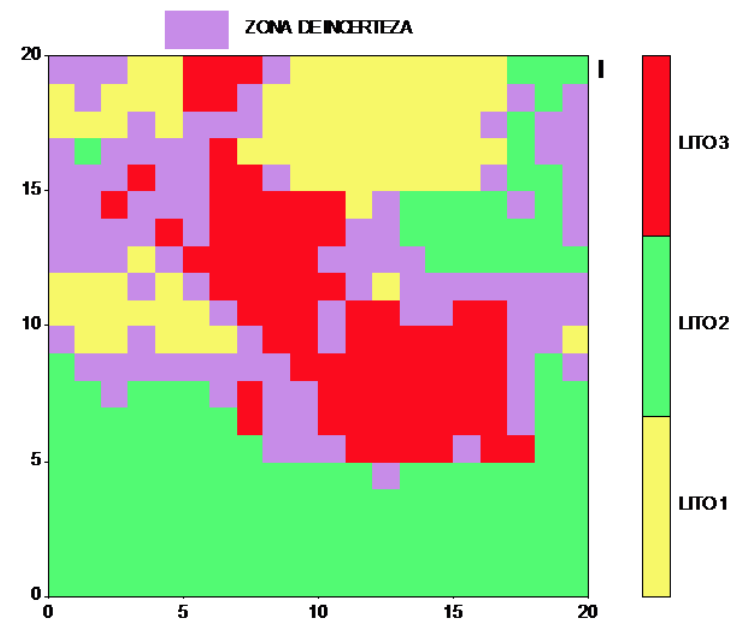

(q)

Rodriguez com R

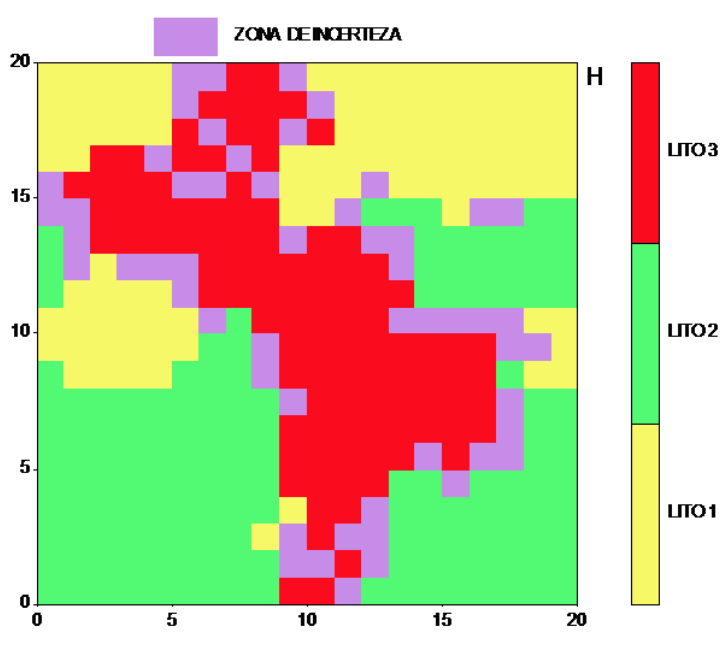

(p)

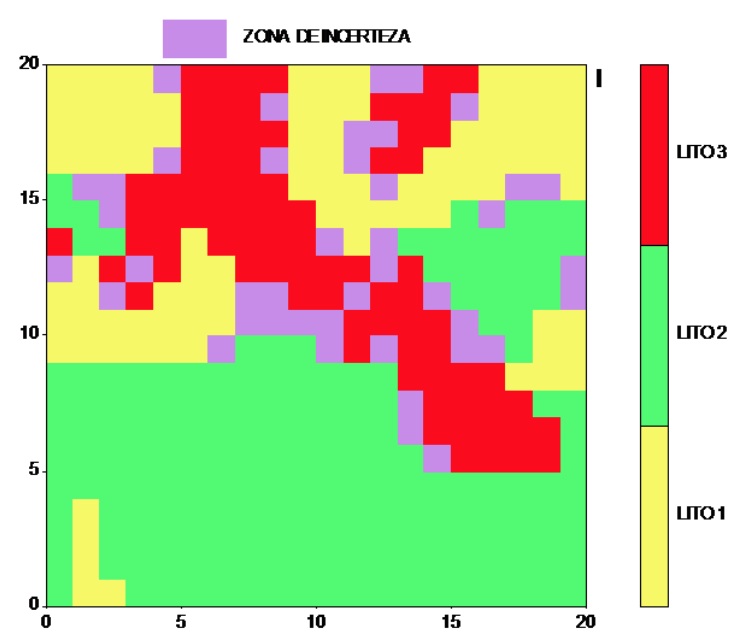

(r)

Figura 6.3: (m) $7^{\circ}$ realização segundo Strebelle, (n) $7^{\circ}$ realização segundo Rodriguez, (o) $8^{\circ}$ realização segundo Strebelle, $(\mathrm{p}) 8^{\circ}$ realização segundo Rodriguez, (q) $9^{\circ}$ realização segundo Strebelle, (r) $9^{\circ}$ realização segundo Rodriguez. 


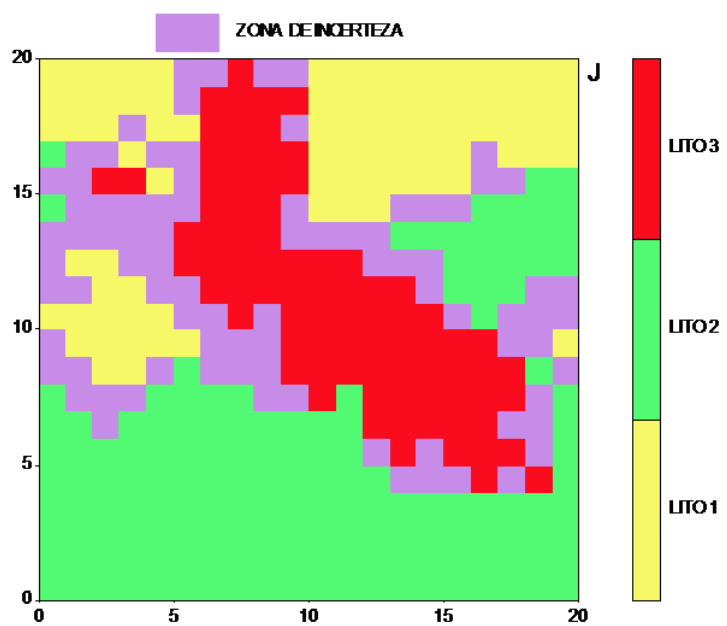

(s)

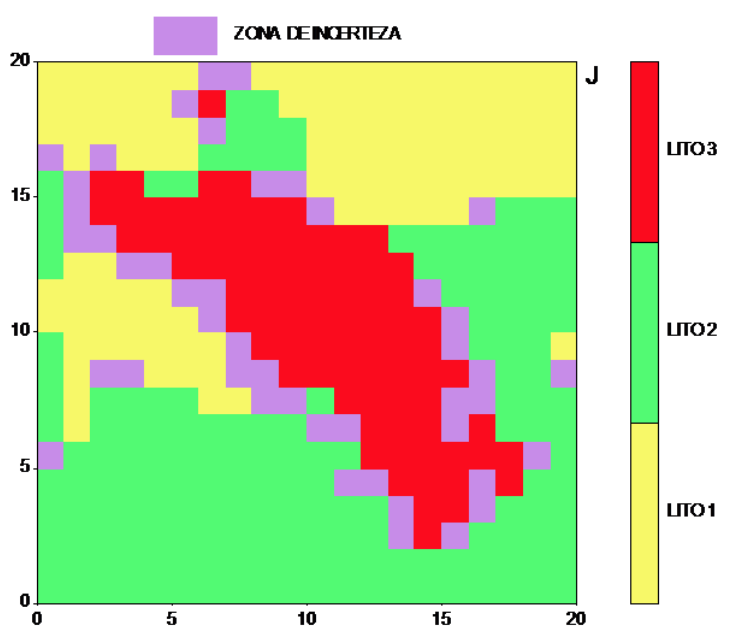

(t)

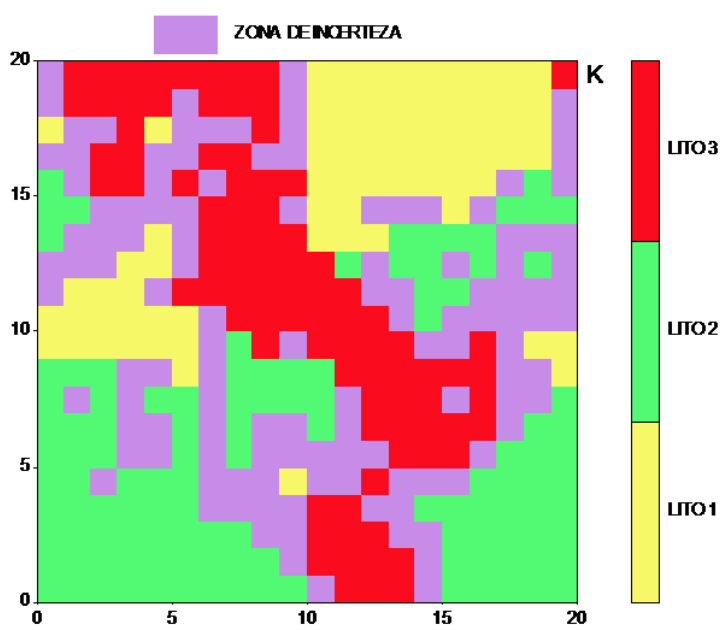

(u)

Rodriguez com R

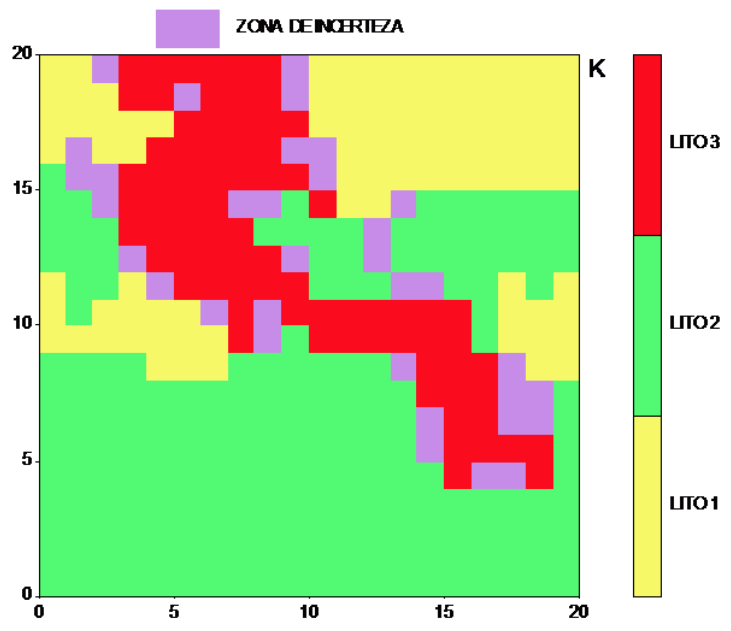

(v)

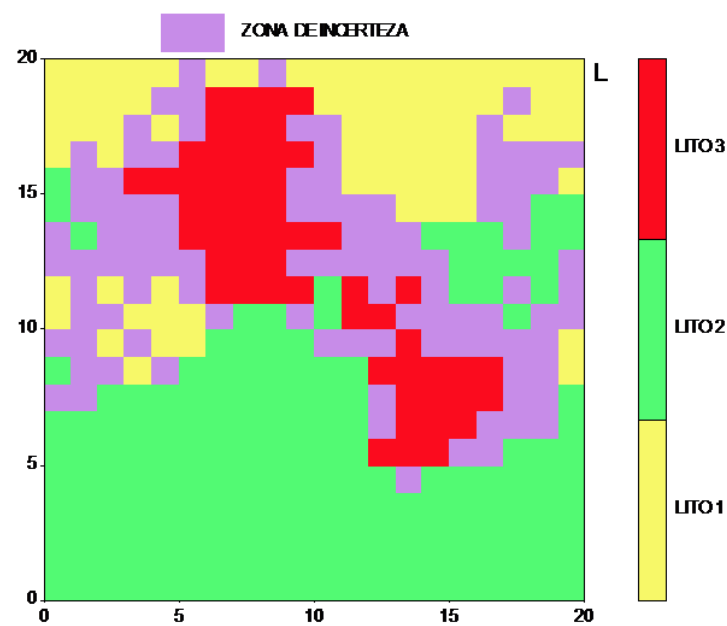

(w)

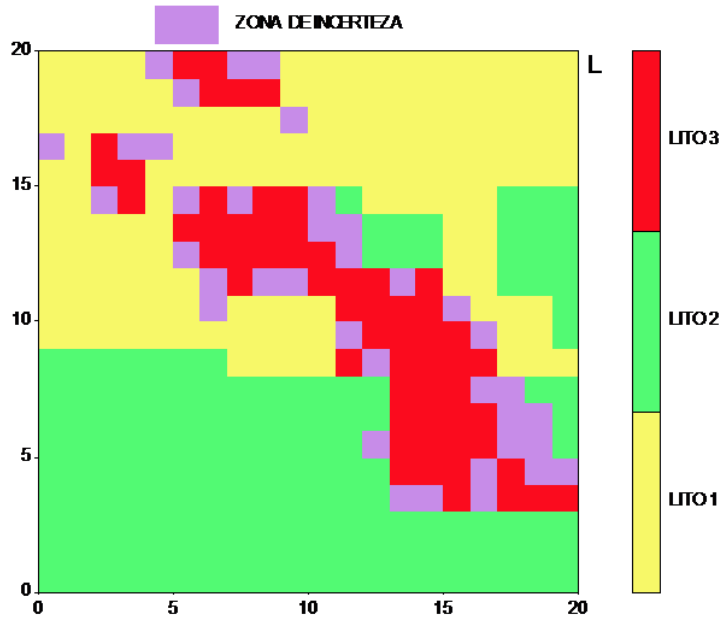

(x)

Figura 6.4: (s) $10^{\circ}$ realização segundo Strebelle, (t) $10^{\circ}$ realização segundo Rodriguez, (u) $11^{\circ}$ realização segundo Strebelle, (v) $11^{\circ}$ realização segundo Rodriguez, (w) $12^{\circ}$ realização segundo Strebelle, (x) $12^{\circ}$ realização segundo Rodriguez. 


\section{Capítulo 7}

\section{Conclusões}

O programa SGeMS ${ }^{1}$ com seu código fonte snesim.h, serviu de referência para a criação de um algoritmo de simulação estocástica escrito no programa $\mathrm{R}$ para o desenvolvimento da inovadora metodologia multi-pontos, que utiliza distribuições de probabilidade local inferida através da imagem de treinamento para variáveis qualitativas e quantitativas discretas. Em contraste à geoestatística clássica de dois pontos (modelo de variograma), o método, reproduz complexos padrões, como a previsão de facies geológicas, propriedades físicas, geofísicas e geoquímicos da subsuperfície de qualquer fenômeno em 2D (pixels) e 3D (voxels), assim como para reservas minerais, reservatórios petrolíferos, entre outros fenômenos com variáveis regionalizadas.

Em alternativa às técnicas baseadas em objetos (voxels), a proposta de aproximação é geral; esta mesma metodologia permite simular qualquer tipo de fenômeno com comportamento heterogêneo, de qualquer forma e em qualquer escala.

Este algoritmo baseado em árvore de busca não necessita ser modificado para estimar um objeto particular e/ou geometria; sua linguagem simples, reproduz qualquer padrão complexo. A razão para tal generalidade é que o usuário não precisa decidir com antecedência quais estatísticas ou parâmetros geoestatísticos são essenciais, pois esses parâmetros serão reproduzidos nas realizações simuladas. Todas as estatísticas são diretamente providenciadas com a imagem de treinamento, portanto, devem exibir as heterogeneidades consideradas relevantes para a subsuperfície. O algoritmo de árvore de busca, segue os passos dos modelos clássicos de modelagem de variogramas e krigagem.

A simplicidade desta metodologia, no entanto, a partir de uma maior dependência sobre a decisão a priori da estacionariedade local, onde serão herdados os valores estimados a partir da imagem de treinamento. A partir de agora, o algoritmo herda estruturas presentes nas imagens de treinamento, sem limites, com o risco de exportação de detalhes irrelevantes, podendo ser repassados a área ou subsuperfície de estudo. Mas, também consegue-se controlar a frequência das propriedades a serem exportadas para a área ou subsuperfície, a imagem de treinamento pode mostrar uma tendência que não é relevante, mantendo apenas

\footnotetext{
${ }^{1}$ Software de modelagem geoestatístico desenvolvido pela Universidade Stanford.
} 
as características de alta frequência que permitem a filtragem dessas tendências. Inversamente, utilizou-se uma imagem suavizada de treinamento gerada por um algoritmo baseado em objeto, apenas as estatísticas de baixa frequência correspondentes aos objetos em grande escala deverá ser reproduzida.

Observa-se uma melhor compreensão da imagem de treinamento, onde pode-se controlar o impacto sobre o fluxo de respostas. Portanto, esta tese demonstra a "essência" da metodologia multi-pontos a ser compreendida, na qual visualiza-se a técnica de identificação sobre os padrões reproduzidos. Observa-se também, uma seleção adequada dos eventos dos dados condicionados ao restringir características não desejáveis produzidas pela metodologia multipontos, porem, que são repassadas a partir da imagem de treinamento, o que implica na plena dependência das restrições geométricas dos dados do evento condicionado.

Conforme os resultados apresentados no capítulo anterior, a metodologia proposta permite obter realizações multi-ponto com incertezas muito menores que aquelas resultantes da metodologia clássica. As realizações da simulação estocástica devem ser acompanhadas de suas incertezas, uma vez que elas são baseadas em dados amostrais e, portanto, sempre sujeitas a incertezas. Evidentemente, no caso da simulação multi-ponto, deve ser considerada a correlação da imagem de treinamento com os dados amostrais, pois se a correlação for baixa pode-se introduzir outra fonte de erro além daquela inerente à amostragem.

Em conclusão, esta pesquisa permitiu obter realizações multi-ponto com menores incertezas, bem como, possibilitou o mapeamento da zona de incerteza que é fundamental para o exame das realizações individuais.

A utilização desta nova ferramenta servirá como base para futuras e diversas aplicações geoestatísticas dentro do conceito da geoestatística multi-pontos e compartilhada mediante o R. Além de contribuir na avaliação de projetos geológicos, geofísicos e petrolíferos, permitirá amparar tecnicamente decisões estratégicas com maior grau de confiança e precisão. O programa e os bancos de dados utilizados podem ser solicitados diretamente via email: joaneime.usp.br.

\subsection{Recomendações}

Assim como na estatística bayesiana, sempre que possível é bom integrar ao modelo preditivo as informações com conhecimento prévio, como é o casso da metodologia multiponto, desta forma, leva a previsões mais precisas. Portanto, quando tiver uma boa imagem de treinamento que reflita o comportamento da área amostral, recomenda-se esta ferramenta, que caracterizará e identificará alvos com menor incerteza. 


\subsection{Sugestões para pesquisas futuras}

Este trabalho oferece várias possibilidades para futuras pesquisas, entre estas destaca-se:

- Resolver problemas com diferentes tipos de template de busca na nova metodologia multi-ponto.

- Desenvolver diversas aplicações em sistemas de dados georreferenciados onde a informação esteja faltando (perdidas).

- Implementar generalização funcional com interface do software estatístico R.

- Desenvolver a metodologia multi-ponto com co-variáveis utilizando a nova proposta desta tese. 


\section{Referências Bibliográficas}

Anterion, F., Eymard, R., e Karcher, B. (1989). Use of parameter gradients for reservoir history matching. In Source SPE Symposium on Reservoir Simulation, Houston, Texas. Society of Petroleum Engineers. 7

Arpat, G. B. (2005). Sequential simulation with patterns. PhD thesis, Stanford University, Stanford, CA. 8

Breiman, L., Friedman, J. H., Olshen, R. A., e Stone, C. J. (1984). Classification and regression trees. Wadsworth and Brooks, Monterey, CA. 8

Caers, J. e Journel, A. G. (1998). Stochastic reservoir simulation using neural networks trained on outcrop data. In Source SPE Symposium on Reservoir Simulation, 49026, New Orleans, Louisiana. Society of Petroleum Engineers. 8

Certes, C. e De Marsily, G. (1991). Application of the pilot point method to the identification of aquifer transmissivities. Advances in Water Resources, 14(5):284-300. 7

Chiles, J.-P. e Delfiner, P. (1999). Geostatistics: Modeling Spatial Uncertainty. New York City. 7

Clark, I. (1979). Practical Geostatistics. Applied Science Publishers, London. 2

David, M. (1977). Geostatistical Ore Reserve Estimation. Elsevier, New York. 2

Deutsch, C. (2002). Geostatistical reservoir modeling. Applied geostatistics series. Oxford University Press. 7

Deutsch, C. V. (1992). Annealing techniques applied to reservoir modeling and the integration of geological and engineering (Well Test) data. PhD thesis, Stanford University, Stanford, CA. 8,20

Deutsch, C. V. e Journel, A. G. (1992). GSLIB: Geostatistical software library and User's Guide. Oxford University Press. revised edition and printed in 06/11/1997. 30

Duarte, O. d. O. (2003). Dicionário Enciclopédico Inglês-Português de Geofísica e Geologia. $2^{a}$ edição Rio de Janeiro: SBGf. 12 
Farmer, C. (1992). Numerical rocks. in p. king (ed.). the mathematical generation of reservoir geology. Technical report, Clarendon Press. 8

Franke, R. (1982). Scattered data interpolation: Tests of some method. Mathematics of Computation, 38(157):181-200. 17

Gómez-Hernández, J., Sahuquillo, A., e Capilla, J. (1997). Stochastic simulation of transmissitivity fields conditional to both transmissivity and piezometric data - 1. theory. Journal of Hydrology, 203:162-174. 7

Gomez-Hernandez, J. (1991). A stochastic approach to the simulation of block fields conductivity upon data measured at a smaller scale. PhD thesis, Stanford University. 30

Goovaerts, P. (1997). Geostatistics for natural resources evaluation. Oxford University Press, page $470.2,7,25$

Guardiano, F. e Srivastava, M. (1993). Multivariate geostatistics: Beyond bivariate moments, volume 1. Geostatistics-Troia, Kluwer Academic Publications, Dordrecht, in a. soares edition. 20, 25

Haldorsen, H. H. e Damsleth, E. (1990). Stochastic modelling. Journal of Petroleum Technology, 4:404-412. 8

Hardy, R. L. (1971). Multiquadric equations of topography and other irregular surfaces. Journal of Geophysical Research, 76:1905-1915. 17

Isaaks, E. e Srivastava, R. (1989). An introduction to applied geostatistics. Oxford University Press, New York. 2

Journel, A. (1989). Fundamentals of Geostatistics in Five Lessons. Short course in geology, v. 8. American Geophysical Union. 12

Journel, A. G. (1974). Geostatistics for conditional simulation of ore bodies. Economic Geology, 69(5):673-687. ix, 6, 7

Journel, A. G. (1982). The Indicator Approach To Estimation Of Spatial Distributions. SME. 15

Journel, A. G. (1983). Nonparametric estimation of spatial distributions. Journal of the International Association for Mathematical Geology, 15(3):445-468. 15

Journel, A. G. e Huijbregts, C. (1978). Mining Geostatistics. Academic Press (London and New York). 2, 7

Kader, G. D. e Perry, M. (2007). Mapping an uncertainty zone between interpolated types of a categorical variable. Journal of Statistics Education, 15(2):1-16. 16 
Krige, D. G. (1951). A statistical approach to some basic mine valuation problems on the witwatersrand. Journal of the Chemical, Metallurgical and Mining Society of South Africa, 52(6):119-139. 6

Krige, D. G. (1966). Two-dimensional weighted moving average trend surfaces for oreevaluation. Journal of the African Institute of Mining and Metallurgy, 66:13-38. ix, 1, 2

LaVenue, A. M. e Pickens, J. (1992). Application of a coupled adjoint sensitivity and kriging approach to calibrate a ground water flow model. Water Resources Research, 6:1543-1569. 7

Leuangthong, O., Khan, K., e Deutsch, C. V. (2008). Solved Problems in Geostatistics. John Wiley \& Sons. 16

Matheron, G. (1962). Traité de géostatistique appliquée, tome i: Mémoires du bureau de recherches géologiques et minières. Pairs: Editions Technip, 14. 6

Matheron, G. (1963). Traité de géostatistique appliquée, tome ii: Le krigeage. mémoires du bureau de recherches geologiques et minieres. 6

Matheron, G. (1965). Les variables régionalisées et leur estimation: Paris. 6, 12

Matheron, G. (1971). The theory of regionalized variables and its applications. Cahiers du Centre de Morphologie Mathématique, Fountoinebleau, 5. 1, 6, 11

Matheron, G. (1973). The intrinsic random functions and their application. Advances in Applied Probability, 5:439-468. 6

Olea, R. A. (1999). Geostatistics for engineers and earth scientists. Kluwer Academic Publishers, Boston. 2

R, C. T. (2013). R: A Language and Environment for Statistical Computing. R Foundation for Statistical Computing, Vienna, Austria. 4, 5, 10, 26, 34, 38, 43, 46, 47, 48, 49, 50, 51, 52

RamaRao, B. S., LaVenue, A. M., De Marsily, G., e Marietta, M. G. (1995). Pilot point methodology for automated calibration of an ensemble of conditionally simulated transmissivity fields: 1. theory and computational experiments. Water Resources Research, 31(3):475-493. 7

Remy, N., Boucher, A., e Wu, J. (2009). Applied Geostatistics with SGeMS: A user ̌s Guide. Cambridge University Press. 25, 28, 30, 36 
Roggero, F. e Hu, L. (1998). Gradual deformation of continuous geostatistical models for history mactching. In of Petroleum Engineers, S., editor, 27-30 September. New Orleans, Louisiana. 8

Srinivasan, S. e Caers, J. (2000). Conditioning reservoir models to dynamic data - a forward modeling perspective. In of Petroleum Engineers, S., editor, Annual Technical Conference and Exhibition. Dallas, Texas. 10

Srivastava, R. M. (1992). Reservoir characterization with probability field simulation. Society of Petroleum Engineers, Paper 24753. 6, 8

Stoyan, D., Kendall, W., e Mecke, J. (1987). Stochastic geometry and its applications. New York. 8

Strebelle, S. (2000). Sequential simulation drawing structure from training images. Phd thesis, Stanford University, Stanford, CA, USA. viii, xi, 20, 21, 22, 28, 29, 31, 32, 34, 35, $36,39,41,43,44,46,47,48,49$

Strebelle, S. (2002). Conditional simulation of complex geological structures using multiplepoint statistics. Mathematical Geology, 34:1-21. 10, 18, 19, 21, 22, 23, 24, 25, 26, 29, 30, 32,36

Tjelmeland, H. (1996). Stochastic Models in Reservoir Characterization and Markov Random Fields for Compact Objects. PhD thesis, Norwegian University of Science and Technology, Trondheim, Norway. 7

Tjelmeland, H. e Besag, J. (1998). Markov random fields with higher-order interactions. Scandinavian Journal of Statistics, 25(3):415-433. ix, 7, 9

Tran, T. T. (1994). Improving variogram reproduction on dense simulation grids. Computers Ef Geosciences, 20(7Ü8):1161-1168. 30

Trangmar, B. B., Yost, R. S., e Uehara, G. (1985). Aplications of geostatistics to espatial studies of soil properties. Advances in Agronomy, Academic Press, 38:45-94. 1

Vieira, S., Nielsen, D., e Biggar, J. (1981). Spatial variability of field-measured infiltration rate. Soil Science Society of America Journal, Oxford, 45:1040-1048. ix, 1, 3

Yamamoto, J. K. (2000). An alternative measure of the reliability of ordinary kriging estimates. Mathematical Geology, 32:489-509. viii, 17, 18, 35

Yamamoto, J. K. (2001). Avaliação e Classificação de Reservas Minerais, volume 38. Editora da Universidade de São Paulo, São Paulo. ix, 14 
Yamamoto, J. K., Mao, X. M., Koike, K., Crosta, A. P., Landim, P. M. B., Hu, H. Z., Wang, C. Y., e Yao, L. Q. (2012). Mapping an uncertainty zone between interpolated types of a categorical variable. Computers \& Geosciences, 31(7):146-152. x, xi, xiv, 17, 18, 34, 35, $36,43,44,45$ 


\section{Código snesim.R por Rodriguez, J.N.C.}

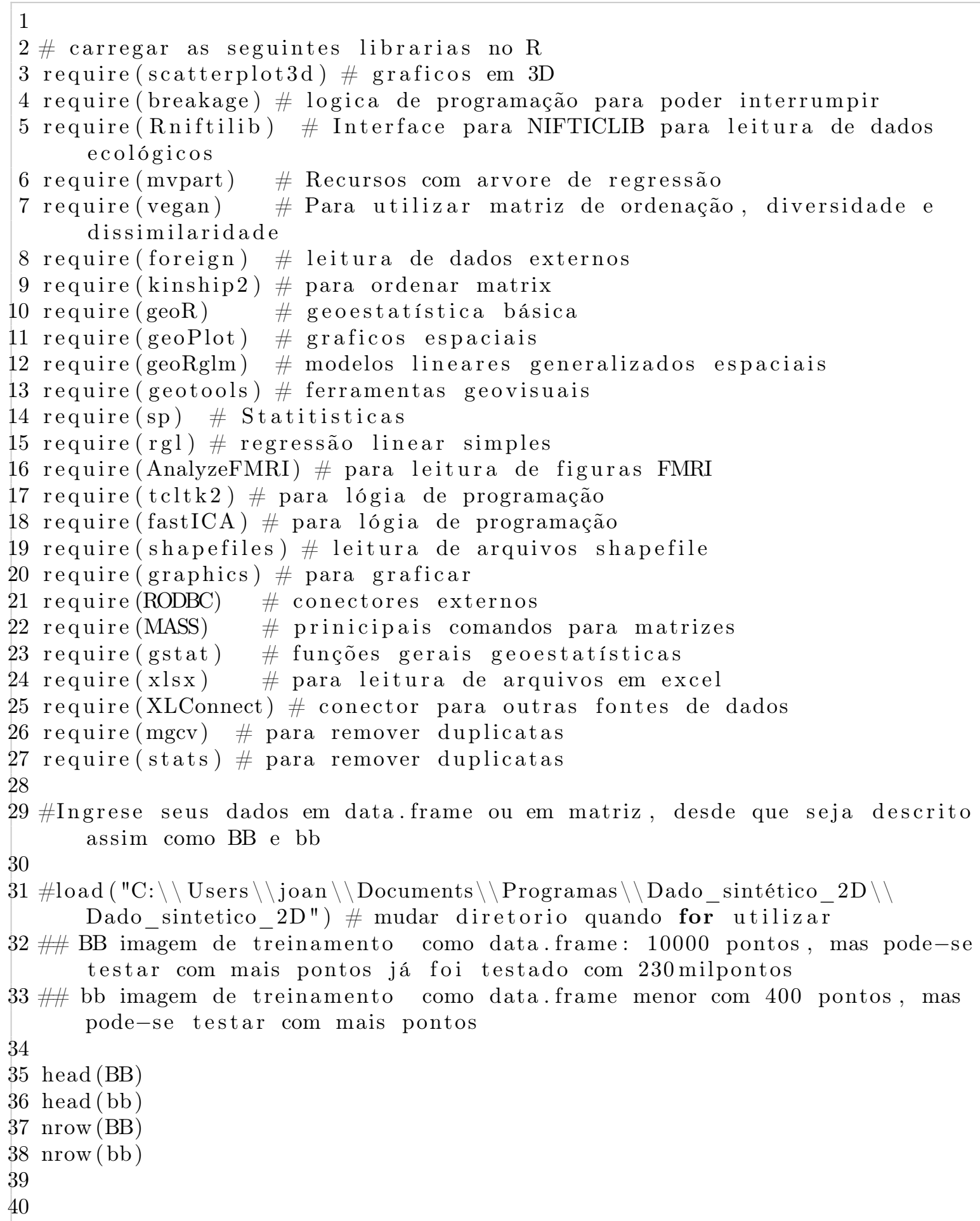


41 if else ( is . matrix $(\mathrm{BB})=\mathrm{TRUE},\{$

$42 \mathrm{BB} 1<$-data.frame $(\mathrm{BB}[, 1], \mathrm{BB}[, 2], \mathrm{BB}[, 3], \mathrm{BB}[, 4]) \quad$ \# realocamos a matriz de dados de treinamento num data frame do $\mathrm{R}$

43 names $(\mathrm{BB} 1)[1]<-$ "i_x" \# renomeamos o nome da coluna de X

44 names $(\mathrm{BB} 1)[2]<-" \mathrm{j}_{-} \mathrm{y} " \quad$ \# renomeamos o nome da coluna de Y

45 names $(\mathrm{BB} 1)[3]<-\quad \mathrm{k}$ z" \# renomeamos o nome da coluna de Z

46 names(BB1) [4] <- "categ" \# renomeamos o nome da coluna das respostas categóricas representadas em $1,2,3, \ldots, \mathrm{K}$. Agora, estuda-se com $\mathrm{K}=3$

47 print ("Sua entrada de dados é matriz, ela será convertido em data frame $\mathrm{BB} 1 ")\}$,

48 if else ( is . data . frame $(\mathrm{BB})=\mathrm{TRUE},\{$

$49 \mathrm{BB} 1<-\mathrm{BB} \quad$ \# realocamos o data frame de dados de treinamento em BB1

50 names $(\mathrm{BB} 1)[1]<-" \mathrm{i} \mathrm{x}^{\prime} \quad$ \# renomeamos o nome da coluna de $\mathrm{X}$

51 names $(\mathrm{BB} 1)[2]<-" \mathrm{j}_{-} \mathrm{y} " \quad$ \# renomeamos o nome da coluna de Y

52 names $(\mathrm{BB} 1)[3]<-$ "k_z" \# renomeamos o nome da coluna de Z

53 names(BB1) [4] - " categ" \# renomeamos o nome da coluna das respostas categóricas representadas em $1,2,3, \ldots, \mathrm{K}$. Agora, estuda-se com $\mathrm{K}=3$

54 print("Sua entrada de dados é data frame, ela será repassado no data frame $\mathrm{BB} 1 ")$

$55\},\{$ print ("Mudar Sua entrada de dados para que o programa comece a

56 )) trabalhar, pode ser formato matriz ou data frame")\}

57

58

59 \# As categorias utilizadas são: $\operatorname{preto}=1$, vermelho $=2$, verde $=3$

60 vec_categ<-sort (unique (BB1[,"categ"])) \# obtem as categorias codificadas em números

61 vec_categ

62

63 \# Tratamento dos dados de entrada

64 \# manter a posição das variáveis:

65 \# X na coluna 1, Y na coluna 2, Z na coluna 3 e categ na coluna 4, os nomes das colunas é indiferentes

$66 \#[, 1] \quad[, 2] \quad[, 3] \quad[, 4]$

$67 \# \quad \mathrm{X} \quad \mathrm{Y} \quad \mathrm{Z} \quad$ categ

68

69

70 \#Verifique se a área é um poligono retangular

71

72

73 \# Entrada de dados da imagem de treinamento: BB

74 \# Entrada de dados reais com coordenadas UTM Datum WGS84 em metros

75

76 \# Definir tamanho dos bloquinhos para as imagens

77 tam_x $<-1 \quad$ \# Exemplo: $50 \mathrm{~m}$

78 tam_y $<-1 \quad$ \# Exemplo: $50 \mathrm{~m}$

79 tam_z $<-1 \quad \#$ Exemplo: $1 \mathrm{~m}$

80

81 \# Definindo ponto inicial da imagem de treinamento

$82 \times 1$ inic $<-\min (\mathrm{BB} 1[, 1]) \quad$ \# mínimo em $\mathrm{X}$

83 y1_inic $<-\min (\mathrm{BB} 1[, 2]) \quad \#$ mínimo em $\mathrm{Y}$

$84 \mathrm{z}^{-}{ }_{-}^{-}$inic $<-\min (\mathrm{BB} 1[, 3]) \quad$ \# mínimo em Z

85 \# $\bar{D}$ efinindo ponto final da imagem de treinamento

$86 \times 1$ final $<-\max (\mathrm{BB} 1[, 1])$ \# máximo em $\mathrm{X}$

87 y1_final $<-\max (\mathrm{BB} 1[, 2])$ \# máximo em $\mathrm{Y}$

$88 \mathrm{z} 1$ _final $<-\max (\mathrm{BB} 1[, 3]) \quad \#$ máximo em Z 
91 \# parâmetro de controle

92 \# Definir o tamanho do bloco

93 tam $\mathrm{x}$ \# $50 \mathrm{~m}$ para nosso casso real

94 tam_y \# $50 \mathrm{~m}$ para nosso casso real

95 tam_z \# $1 \mathrm{~m}$ para nosso casso real

96

97 \# Ponto inicial da imagem de treinamento

$98 \times 1$ inic \# coordenadas inicial em $\mathrm{X}$

99 y 1 inic \# coordenadas inicial em Y

100 z1_inic \# coordenadas inicial em Z

101

102 \# Ponto inicial da imagem de treinamento

$103 \times 1$ final \# coordenadas final em $X$

104 y1_final \# coordenadas final em $Y$

$105 \mathrm{z}^{-}$final \# coordenadas final em Z

106

107 \# Calcula-se o número de bloquinhos em $\mathrm{X}, \mathrm{Y}$ e Z

$108 \mathrm{nx}<-(\mathrm{x} 1$ final-x1_inic $) \% / \%$ tam_x $+1 \quad$ \# calcula-se o número de bloquinhos em $\overline{\mathrm{X}}$

109 ny $<-(y 1$ final-y1_inic $) \% / \%$ tam_y +1 \# calcula-se o número de bloquinhos em $\overline{\mathrm{Y}}$

$110 \mathrm{nz}<-(\mathrm{z} 1$ final-z1_inic $) \% / \%$ tam_z +1 \# calcula-se o número de bloquinhos

em $\bar{Z}$, quando nz é 1 estamos em duas dimensões 2D

111

112 nx \# número de bloquinhos em X

113 ny \# número de bloquinhos em Y

$114 \mathrm{nz}$ \# número de bloquinhos em Z, se nz é 1 estamos em duas dimensões 2D

115 nxyz $<-\mathrm{nx} * \mathrm{ny} * \mathrm{nz} \quad \#$ calcula - se o número total de bloquinhos em estudo

116 nxyz \# número total de bloquinhos em estudo na imagem de treinamento

117

118 \# Entrada de dados das amostras

119 \# Estuda-se como imagem de saída, uma amostra de uma imagem controlada para garantir a análise de diagnóstico da metodologia multiponto

120

121 \# Imagem de treinamento será definido como: Imagem_treino<-bb1

122 \# para isto, define-se um vetor aleatorio para selecionar amostras no data frame bb

123 \# para controlar o arranjo aleatorio utilizamos a função set.seed(número de controle)

124

125 set.seed(3456) \# função que controla o arranjo aleatório em "3456", é para gerar a mesma amostra apresentada na tese

126 bb1<-data.frame (bb [sample $(1:$ nrow $(b b), 25)$,$] , row names =$ NULL $)$ \# escolhe-se o tamanho das amostras para testar a metodologia multiponto em $25(6.25 \%), 50(12.5 \%)$ e $150(37.5 \%)$

127 open3d() \# função para preparar o ambiente de gráficos

128 plot3d(bb1[,1], bb1 [,2], bb1[,3], col=bb1[,4], type='s', radius=c $(0.55,0.55,0.55), x l a b=" X ", y l a b=" Y "$, zlab="Z") \# função que gráfica em $3 \mathrm{D}$

129

130

131 \# Definindo ponto inicial da imagem de saida

$132 \mathrm{x} 1$ inic_s $<-\min (\mathrm{bb1}[, 1])$ \# mínimo em $\mathrm{X}$ da imagem de saída, verificar o mínimo em $\mathrm{X} / \mathrm{Y} / \mathrm{Z}$, pois a amostra não necessariamente irá conter mínimo desejado na coordenada $\mathrm{X} / \mathrm{Y} / \mathrm{Z}$

133 y1_inic_s $<-\min (b b 1[, 2]) \quad \#$ mínimo em $\mathrm{Y}$ da imagem de saída

134 z1_inic_s $<-\min (\mathrm{bb} 1[, 3]) \quad$ \# mínimo em $\mathrm{Z}$ da imagem de saída

135 \# Definindo ponto final da imagem de saida 
136 x1_final_s $<-\max (\mathrm{bb} 1[, 1])$ \# máximo em $\mathrm{X}$ da imagem de saída, verificar o máximo em $\mathrm{X} / \mathrm{Y} / \mathrm{Z}$, pois a amostra não necessariamente irá conter o máximo desejado na coordenada $\mathrm{X} / \mathrm{Y} / \mathrm{Z}$

137 y1_final_s $<-\max (\mathrm{bb} 1[, 2])$ \# máximo em Y da imagem de saída

138 z1_final_s $<-\max (\mathrm{bb} 1[, 3])$ \# máximo em Z da imagem de saída

139

$140 \mathrm{x} 1$ inic_s \# coordenadas inicial em $\mathrm{X}$ da imagem de saída

141 y1_inic_s \# coordenadas inicial em Y da imagem de saída

142 z1 inic $\mathrm{s}$ \# coordenadas inicial em Z da imagem de saída

143

144 \# Ponto inicial da imagem de treinamento

145 x1_final_s \# coordenadas final em $\mathrm{X}$ da imagem de saída

146 y1_final_s \# coordenadas final em Y da imagem de saída

147 z1_final_s \# coordenadas final em Z da imagem de saída

148

149 \# O tamanho dos bloquinhos a priori tem que ser o mesmo que a imagem de treinamento

150 \# Calcula-se o número de bloquinhos em $\mathrm{X}$, Y e Z da imagem de saída

151 nx_s $<-\left(\mathrm{x} 1\right.$ final_s $-\mathrm{x} 1{ }_{-}$inic_s $) \% / \%$ tam_x $+1 \quad \#$ calcula-se o número de bloquinhos em $\mathrm{X}$

152 ny_s $<-(y 1$ final_s $-y 1$ inic_s $) \% / \%$ tam_y $+1 \quad \#$ calcula - se o número de bloquinhos em Y

153 nz_s $<-(z 1$ final_s-z1_inic_s $) \% / \%$ tam_z $+1 \quad$ \# calcula - se o número de bloquin̄hos em Z, quando nz é 1 estamos em duas dimensões 2D

154

155 \# precisamos saber o tamanho do cubo de dados da imagem de treinamento 156

157 nx_s \# número de bloquinhos em X na imagem de saida

158 ny_s \# número de bloquinhos em Y na imagem de saida

159 nz_s \# número de bloquinhos em Z na imagem de saida, como nz é 1 estamos em duas dimensões 2D

160 nxyz_s $<-n x \_s * n y \_s * n z \_s \quad \#$ calcula-se o número total de bloquinhos em estudo na imagem de saida

161 nxyz_s \# número total de bloquinhos em estudo na imagem de treinamento 162

163 nrow(bb1) \# quantidade de informações na imagem de saída, neste casso equivalente a $25(6.25 \%)$ ou $50(12.5 \%)$ ou $150(37.5 \%)$

164

165

166 \# Construindo o template de busca

167 \# escolhe-se um template em cruz, assim como pode-se escolher muitos outros 12 arranjos distintos

$168 \mathrm{M} 1<-$ matrix $(\mathrm{c}($

$1690,1,0, \quad \#(0,1,0) \quad$ é $\mathrm{h} 1$, o vetor que levará até a posição u1=u+h1 para avaliar a resposta da variável em $\mathrm{S}(\mathrm{u} 1)$

$1701,0,0, \quad \#(1,0,0) \quad$ é h2, o vetor que levará até a posição u1=u+h2 para avaliar a resposta da variável em $\mathrm{S}(\mathrm{u} 2)$

$1710,-1,0, \quad \#(0,-1,0)$ é h3, o vetor que levará até a posição u1=u+h3 para avaliar a resposta da variável em S(u3)

$172-1,0,0, \quad \#(-1,0,0)$ é $\mathrm{h} 4$, o vetor que levará até a posição u1=u+h4 para avaliar a resposta da variável em $\mathrm{S}(\mathrm{u} 4)$

$1731,1,0, \quad \#(1,1,0) \quad$ é $\mathrm{h} 5$, o vetor que levará até a posição u1=u+h5 para avaliar a resposta da variável em S( 45$)$

$1741,-1,0, \quad \#(1,-1,0)$ é h6, o vetor que levará até a posição u $1=u+h 6$ para avaliar a resposta da variável em $\mathrm{S}(\mathrm{u} 6)$

$175-1,-1,0$, \# $(-1,-1,0)$ é h7, o vetor que levará até a posição u1=u+h7 para avaliar a resposta da variável em $\mathrm{S}(\mathrm{u} 7)$

$176-1,1,0, \#(-1,1,0)$ é h8, o vetor que levará até a posição u $1=\mathrm{u}+\mathrm{h} 8$ para avaliar a resposta da variável em S(u8) 
$1770,2,0, \quad \#(0,2,0) \quad$ é $\mathrm{h} 9$, o vetor que levará até a posição u1=u+h9 para avaliar a resposta da variável em $\mathrm{S}(\mathrm{u} 9)$

$1780,-2,0, \quad \#(0,-2,0)$ é $\mathrm{h} 10$, o vetor que levará até a posição u1=u+h10 para avaliar a resposta da variável em $\mathrm{S}(\mathrm{u} 10)$

$1792,0,0, \quad \#(2,0,0)$ é h11, o vetor que levará até a posição u1=u+h11 para avaliar a resposta da variável em $\mathrm{S}(\mathrm{u} 11)$

$180-2,0,0$ \# $(-2,1,0)$ é h12, o vetor que levará até a posição u1=u+h12 para avaliar a resposta da variável em $\mathrm{S}(\mathrm{u} 12)$

181 ) $, 12,3$, byrow=TRUE) \# os vetores formam uma matriz de 12 linhas $\mathrm{x} 3$ colunas

182

183

184 \# Definimos Valores NA para verificar o valor da categoria na localização u segundo o template que irá ter 12 vetores

185

$186 \mathrm{u} 1<-\mathrm{u} 2<-\mathrm{u} 3<-\mathrm{u} 4<-\mathrm{u} 5<-\mathrm{u} 6<-\mathrm{u} 7<-\mathrm{u} 8<-\mathrm{u} 9<-\mathrm{u} 10<-\mathrm{u} 11<-\mathrm{u} 12<-\mathrm{NA}$

187 nM1<-nrow(M1) \# é o número de níveis realacionado ao template de busca com 12 níveis na arvore de probabilidades condicionais

188

189 \# a imagem de treinamento será alocada na matriz "Imagem_treino"

190 \# preparando a tabela para calcular as probabilidades condicionais

191 Imagem_treino<-data.frame $(\mathrm{BB} 1[, 1], \mathrm{BB} 1[, 2], \mathrm{BB} 1[, 3], \mathrm{BB} 1[, 4], \mathrm{u} 1, \mathrm{u} 2, \mathrm{u} 3, \mathrm{u} 4, \mathrm{u} 5$ $, \mathrm{u} 6, \mathrm{u} 7, \mathrm{u} 8, \mathrm{u} 9, \mathrm{u} 10, \mathrm{u} 11, \mathrm{u} 12)$ \# o objeto "Imagem_treino" recebe os valore

do data frame BB1 (imagem de treinamento)

192 names (Imagem_treino) [1] <- "i_x" \# renomeamos o nome da coluna de $\mathrm{X}$

193 names (Imagem_treino) $[2]<-" j_{-}{ }^{-}$" \# renomeamos o nome da coluna de Y

194 names (Imagem_treino) [3] <- "k_z" \# renomeamos o nome da coluna de Z

195 names (Imagem_treino) [4] - " categ" \# renomeamos o nome da coluna das respostas categóricas representadas em $1,2,3, \ldots, \mathrm{K}$. Agora, estuda-se $\operatorname{com~} \mathrm{K}=3$

196

197

198 n_imag_t<-nrow(Imagem_treino) \# é o numero de linhas que o template passara procurando padrões

199

200 \# Mapeamento das probabilidades condicionais em cada bloquinho da imagem de treinamento

201 for $\left(\mathrm{i}\right.$ in $1: \mathrm{n}_{-}$imag_t $)\{$

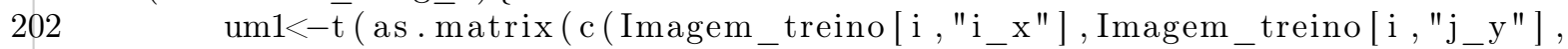

Imagem_treino $\left.\left.\left.\left[\mathrm{i}, " \mathrm{k}_{-} \mathrm{z}^{\prime}\right]\right)+\mathrm{M} 1[1],\right)\right)$ \# captura a posição do vector

h1 no $\overline{\mathrm{i}}$-ésimo bloquinho, desta forma se obtem o valor de $\mathrm{S}(\mathrm{u} 1)$ um2<-t (as . matrix (c (Imagem_treino [i, "i_x"], Imagem_treino [i , "j_y"],

Imagem_treino [i, "k_z"] $+\mathrm{M} 1[2])$,$) \# captura a posição do vector$ h2 no $\bar{i}$-ésimo bloquinho, desta forma se obtem o valor de $\mathrm{S}(\mathrm{u} 2)$ um3<-t (as . matrix (c (Imagem_treino [i, "i_x"], Imagem_treino [i, "j_y"],

Imagem_treino $\left.\left.\left.\left[\mathrm{i}, " \mathrm{k}_{-} \mathrm{z}^{\prime}\right]\right)+\mathrm{M} 1[3],\right)\right)$ \# captura a posição do vector h3 no $\overline{\mathrm{i}}$-ésimo bloquinho, desta forma se obtem o valor de $\mathrm{S}(\mathrm{u} 3)$ um4<-t (as . matrix (c (Imagem_treino [i , "i_x"], Imagem_treino [i , "j_y"],

Imagem_treino [i, "k_z"]) $+\mathrm{M} 1[4]$,$) ) \# captura a posição do vector$ h4 no $\overline{\mathrm{i}}$-ésimo bloquinho, desta forma se obtem o valor de $\mathrm{S}(\mathrm{u} 4)$ um5<-t (as . matrix (c (Imagem_treino [i , "i_x"], Imagem_treino [i , "j_y" ],

Imagem_treino $\left.\left.\left.\left[\mathrm{i}, " \mathrm{k} \_\mathrm{z}^{\prime}\right]\right)+\mathrm{M} 1[5],\right)\right)$ \# captura a posição do vector h5 no $\overline{\mathrm{i}}$-ésimo bloquinho, desta forma se obtem o valor de $\mathrm{S}(\mathrm{u} 5)$ um6<-t (as . matrix (c (Imagem_treino [i, "i_x"], Imagem_treino [i, "j_y"],

Imagem_treino $\left.\left.\left.\left[\mathrm{i}, " \mathrm{k}_{-} \mathrm{z}^{\prime}\right]\right)+\mathrm{M} 1[6],\right)\right)$ \# captura a posição do vector h6 no $\overline{\mathrm{i}}$-ésimo bloquinho, desta forma se obtem o valor de $\mathrm{S}(\mathrm{u} 6)$ um7<-t (as . matrix (c (Imagem_treino [i , "i_x"], Imagem_treino[i , "j_y"],

Imagem_treino [i,"k_z"])+M1[7,])) \# captura a posição do vector h7 no $\overline{\mathrm{i}}$-ésimo bloquinho, desta forma se obtem o valor de $\mathrm{S}(\mathrm{u} 7)$ 
um8<-t (as.matrix (c(Imagem_treino[i, "i_x"], Imagem_treino[i , "j_y"], Imagem_treino $\left.\left.\left.\left[i, " k \_z "\right]\right)+\mathrm{M} 1[8],\right)\right)$ \# captura a posição do vector h8 no $\bar{i}$-ésimo bloquinho, desta forma se obtem o valor de $\mathrm{S}(\mathrm{u} 8)$ um9<-t (as . matrix (c (Imagem treino[i, "i_x"], Imagem treino[i , $\left.j \_y "\right]$, Imagem_treino[i,"k_z"] $+\mathrm{M} 1[9])$,$) \# captura a posição do vector$ h9 no i-ésimo bloquinho, desta forma se obtem o valor de S(u9) Imagem treino[i,"k $\left.\left.\left.\mathrm{z}^{\prime \prime}\right]\right)+\overline{\mathrm{M}} 1[10,1)\right)$ \# captura a posição do vector h10 no $\overline{\mathrm{i}}$-ésimo bloquinho, desta forma se obtem o valor de $\mathrm{S}(\mathrm{u} 10)$ um $11<-$ t (as . matrix (c (Imagem_treino [i , "i_x"], Imagem_treino [i, "j_y" ] , Imagem_treino[i, "k_z"])+M1[11,])) \# captura a posição do vector h11 no $\overline{\mathrm{i}}$-ésimo bloquinho, desta forma se obtem o valor de $\mathrm{S}(\mathrm{u} 11)$ um12<-t (as . matrix (c (Imagem_treino [i , "i_x"], Imagem_treino [i, "j_y"], Imagem_treino $\left.\left.\left.\left[\mathrm{i}, " \mathrm{k} \_\mathrm{z}\right]\right)+\overline{\mathrm{M}} 1[12],\right)\right)$ \# captura a posição do vector h12 no $\overline{\mathrm{i}}$-ésimo bloquinho, desta forma se obtem o valor de $\mathrm{S}(\mathrm{u} 12)$ \# incremente aqui quando aumentar mais vetores para o template de busca

$\mathrm{a}<-$ as .vector $\left(\operatorname{Imagem}\right.$ treino [which $\left(\operatorname{Imagem}\right.$ treino $\left[, " \mathrm{i}_{-} \mathrm{x}^{\prime \prime}\right]==\mathrm{um} 1[1,1] \&$ Imagem_treino $\left[, " j_{-} \mathrm{y}^{\prime \prime}\right]==\mathrm{um} 1[1,2] \&$ Imagem_treino $\left[, " \mathrm{k}_{-} \mathrm{z} "\right]==\mathrm{um} 1$ $[1,3]), "$ categ"]) \# captura o valor do vetor h1 no $\overline{\mathrm{i}}$-ésimo bloquinho

ifelse $(\operatorname{length}(\mathrm{a})==0, \mathrm{a} 1<-\mathrm{NA}, \mathrm{a} 1<-\mathrm{a}) \#$ validando existência

$\mathrm{b}<-$ as . vector (Imagem_treino [which (Imagem_treino [, "i_x" $]==\mathrm{um} 2[1,1] \&$ Imagem_treino $\left[, " \mathrm{j}_{-} \mathrm{y} "\right]==\mathrm{um} 2[1,2] \&$ Imagem_treino $\left[, " \mathrm{k}_{-} \mathrm{z}^{\mathrm{N}}\right]=\mathrm{um} 2$ $[1,3]), "$ categ"]) \# captura o valor do vetor h2 no $\overline{\mathrm{i}}$-ésimo bloquinho

ifelse $(\operatorname{length}(\mathrm{b})==0, \mathrm{~b} 1<-\mathrm{NA}, \mathrm{b} 1<-\mathrm{b}) \quad \#$ validando existencia

$\mathrm{c}<-$ as . vector (Imagem_treino [which (Imagem_treino $\left[, " \mathrm{i}_{-} \mathrm{x}^{\prime \prime}\right]==\mathrm{um} 3[1,1] \&$ Imagem_treino $\left[, " \mathrm{j}_{-} \mathrm{y} "\right]==\mathrm{um} 3[1,2] \&$ Imägem_treino $\left[, " \mathrm{k}_{-} \mathrm{z}\right]==\mathrm{um} 3$ $[1,3]), "$ categ"]) \# captura o valor do vetor h3 no $\overline{\mathrm{i}}$-ésimo bloquinho

ifelse $(\operatorname{length}(\mathrm{c})=0, \mathrm{c} 1<-\mathrm{NA}, \mathrm{c} 1<-\mathrm{c}) \#$ validando existência

$\mathrm{d}<-$ as . vector (Imagem_treino [which (Imagem_treino [, "i_x" $]==\mathrm{um} 4[1,1] \&$ Imagem_treino $\left[, " \mathrm{j}_{-} \mathrm{y}^{\prime \prime}\right]==\mathrm{um} 4[1,2] \quad \&$ Imagem_treino $\left[, " \mathrm{k}_{-} \mathrm{z}^{\prime \prime}\right]==\mathrm{um} 4$ $[1,3]), "$ categ"]) \# captura o valor do vetor h4 no $\overline{\mathrm{i}}$-ésimo bloquinho

ifelse $(\operatorname{length}(\mathrm{d})==0, \mathrm{~d} 1<-\mathrm{NA}, \mathrm{d} 1<-\mathrm{d}) \#$ validando existência

$\mathrm{e}<-$ as . vector (Imagem_treino [which (Imagem_treino [, "i_x"] $==$ um5 [1, 1] \& Imagem_treino $\left[, " \mathrm{j}_{-} \mathrm{y}^{\mathrm{N}}\right]==\mathrm{um} 5[1,2] \&$ Imagem_treino $\left[, " \mathrm{k}_{-} \mathrm{z} "\right]==\mathrm{um} 5$ $[1,3]), \bar{c}$, categ"]) \# captura o valor do vetor h5 no $\overline{\mathrm{i}}$-ésimo bloquinho

ifelse $(\operatorname{length}(\mathrm{e})==0, \mathrm{e} 1<-\mathrm{NA}, \mathrm{e} 1<-\mathrm{e}) \#$ validando existência

$\mathrm{f}<-$ as . vector (Imagem_treino [which (Imagem_treino [, "i_x"] $==$ um6 [1, 1] \& Imagem_treino $\left[, " \mathrm{j}_{-} \mathrm{y} "\right]==\mathrm{um} 6[1,2] \&$ Imagem_treino $\left[, " \mathrm{k}_{-} \mathrm{z}^{\mathrm{N}}\right]==\mathrm{um} 6$ $[1,3])$, "categ"]) \# captura o valor do vetor h6 no $\overline{\mathrm{i}}$-ésimo bloquinho

ifelse $(\operatorname{length}(\mathrm{f})==0, \mathrm{f} 1<-\mathrm{NA}, \mathrm{f} 1<-\mathrm{f})$ \# validando existência

$\mathrm{g}<-$ as . vector (Imagem_treino [which (Imagem_treino [, "i_x"]==um7[1,1] \& Imagem_treino $\left[, " \mathrm{j}_{-} \mathrm{y} "\right]==\mathrm{um} 7[1,2] \&$ Imägem_treino $\left[, " \mathrm{k}_{-} \mathrm{z}^{\prime \prime}\right]==\mathrm{um} 7$ $[1,3]), \bar{c}$, categ"]) \# captura o valor do vetor h7 no $\overline{\mathrm{i}}$-ésimo bloquinho

ifelse $(\operatorname{length}(\mathrm{g})==0, \mathrm{~g} 1<-\mathrm{NA}, \mathrm{g} 1<-\mathrm{g}) \quad \#$ validando existência 
271 \# salve a matriz de probabilidades para reutilizar para outros testes 272 \#save . image ( $\mathrm{C}: \backslash \backslash$ Users $\backslash \backslash$ joan $\backslash \backslash$ Documents $\backslash \backslash$ Programas $\backslash \backslash$ Dado_sintético_2D $\backslash \backslash$ figuras $\backslash \backslash$ Colocar_na_tese $\backslash \backslash$ matriz_deprobabilidades" )

273 \#load ("C: $\backslash \backslash$ Users $\backslash \backslash$ joan $\backslash \backslash$ Documents $\backslash \backslash$ Programas $\backslash \backslash$ Dado_sintético_2D $\backslash \backslash$ figuras $\backslash \backslash$ Colocar_na_tese $\backslash \backslash$ matriz_deprobabilidades")

275 \# repasando as amostras para a matriz AA 
277 AA<-matrix (NA, nxyz_s,42) \# quando precisar de mais colunas pode utilizar , atualmente fixei em 30 colunas, será necessário quando incrementar os votores do template de busca

278 cont $<-0$ \#contador para construir a matriz

279 \# construimos a matriz de saida, que receberá todos os calculos realizados pela estatística multiponto

280 for $\left(\mathrm{k}\right.$ in $\left.1: \mathrm{nz} \_\mathrm{s}\right)\{$

281 for $(\mathrm{j}$ in $1:$ ny_s $)\{$

$282 \quad$ for $\left(\mathrm{i}\right.$ in $\left.1: \mathrm{nx}_{-} \mathrm{s}\right)\{$

$283 \operatorname{cont}<-\operatorname{con} \mathrm{t}+1$

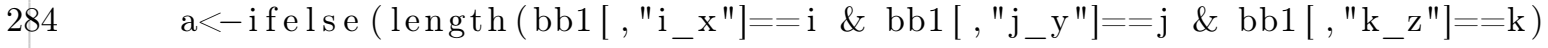
$>=1$

285 array (bb1 [which (bb1 $\left[, " i_{-}{ }^{\prime \prime}\right]==\mathrm{i} \& \operatorname{bb} 1\left[, " j_{-} y "\right]==\mathrm{j} \& \operatorname{bb} 1[$," $\left.\left.\left.\left.\left.\mathrm{k} \_\mathrm{z}^{\prime \prime}\right]==\mathrm{k}\right), 4\right]\right), \mathrm{NA}\right)$

286 AA[cont, 1$]<-\mathrm{i} \quad$ \# a coluna 1 da matriz AA recebe a coordenada em X

287 AA[cont 2$]<-\mathrm{j} \quad$ \# a coluna 2 da matriz AA recebe a coordenada em Y

288 AA[cont, 3$]<-\mathrm{k} \quad$ \# a coluna 3 da matriz AA recebe a coordenada em $Z$

$289 \quad \mathrm{AA}[\operatorname{cont}, 4]<-\mathrm{a}$

$290 \quad$ AA $[$ cont, 5$]<-(\mathrm{k}-1) * \mathrm{nx} \_\mathrm{s} * \mathrm{ny} \_\mathrm{s}+(\mathrm{j}-1) * \mathrm{nx} \_\mathrm{s}+\mathrm{i}$

291

292

293

294

295

296 AA<-AA[order $(\mathrm{AA}[, 5])$,$] \#ordenamos a matriz de saida AA onde receberá todos$ os cálculos

297 \#head (AA)

298

299 AA_antes $<-$ AA \# guardamos a matriz AA em AA_antes antes de começar qualquer cálculo \#AA<-AA_antes

300

301 vetor_e<-as.vector ( AA [which(is.na(AA [,4])=TRUE) ,5] ) \# vetor que contem as posições que precisam receber estimativas na matriz de cálculo AA

302

303 vetor_amostras<-as.vector ( $\mathrm{AA}[$ which (is.na $(\mathrm{AA}[, 4]) !=\mathrm{TRUE}), 5]) \quad \#$ vetor que contem as posições das amostras na matriz de cálculo AA

304

305 posicao_a $<$-vetor_e \# arranjo aleatório

306

307 vetor_e \# mostra o vetor que contem as posições que precisam receber estimativas na matriz de cálculo AA

308 length (vetor_e )

309

310 vetor_amostras \# vetor que contem as posições das amostras na matriz de cálculo AA

311 length (vetor_amostras)

312

313 conta_sem $<-$ conta $0<-$ conta $1<-$ conta $2<-$ conta $3<-$ conta $4<-$ conta $5<-$ conta $6<-$ conta 7 $<-$ conta $8<-$ conta $9<-$ conta $10<-$ conta $11<-$ conta $12<-$ conta $13<-$ conta $14<-0$

316 \# inicio do while enquanto

317

318 while (length (AA [ which (is . na (AA $[, 4])=$ TRUE) , 4])>0) \{

320 posicao_a $<-$ sample $($ posicao_a $)$

322 if else (is .na(AA[posicao_a [1] ,4]) =TRUE, \{ 
324 um<-matrix (NA,nM1,3) \# criação da matriz que recebe as novas posições do bloquinho posicionado em u

325 um1<-matrix (NA,nM1,1) \# criação da matriz um para receber os valores das observações vizinhas

326

327

328

330

331

332

333

334

335

336

337

338

339

340

341

for $(\mathrm{w}$ in 1:nM1) \{

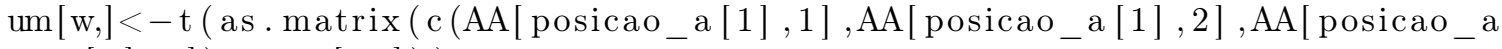
$[1], 3])+\mathrm{M} 1[\mathrm{w}])$,$) \# matriz um recebe os vectores de coordenadas que$ fazem parte dos vizinhos segundo o template de busca

$\mathrm{um} 1[\mathrm{w}, 1]<-(\mathrm{um}[\mathrm{w}, 3]-1) * \mathrm{nx} \_\mathrm{s} * \mathrm{ny} \_\mathrm{s}+(\mathrm{um}[\mathrm{w}, 2]-1) * \mathrm{nx} \_\mathrm{s}+\mathrm{um}[\mathrm{w}, 1] \#$ matriz um recebe as observações (categorias codificadas em 1,2,3) nas coordenadas que fazem parte dos vizinhos segundo o template de busca \}

\# Alocando e resgatando os valores em $\mathrm{S}(\mathrm{u}+\mathrm{h})$

$\mathrm{S}<-\mathrm{c}()$

if els e $(\operatorname{length}(\mathrm{AA}[$ which $(\mathrm{AA}[, 5]==\operatorname{um} 1[1,1]), 4])==0, \mathrm{~S}[1]<-\mathrm{NA}, \mathrm{S}[1]<-\mathrm{AA}[\mathrm{which}($ $\mathrm{AA}[, 5]==\operatorname{um} 1[1,1]), 4]) \quad$ \#captura o valor da variável $\mathrm{S}(\mathrm{u} 1)$

if els e $(\operatorname{length}(\mathrm{AA}[$ which $(\mathrm{AA}[, 5]==\operatorname{um} 1[2,1]), 4])==0, \mathrm{~S}[2]<-\mathrm{NA}, \mathrm{S}[2]<-\mathrm{AA}[$ which ( $\mathrm{AA}[, 5]==\operatorname{um} 1[2,1]), 4]) \quad$ \#captura o valor da variável $\mathrm{S}(\mathrm{u} 2)$

if els e $(\operatorname{length}(\mathrm{AA}[$ which $(\mathrm{AA}[, 5]==\operatorname{um} 1[3,1]), 4])==0, \mathrm{~S}[3]<-\mathrm{NA}, \mathrm{S}[3]<-\mathrm{AA}[\mathrm{which}($ $\mathrm{AA}[, 5]==\operatorname{um} 1[3,1]), 4]) \quad$ \#captura o valor da variável $\mathrm{S}(\mathrm{u} 3)$

if els e (length $(\mathrm{AA}[$ which $(\mathrm{AA}[, 5]==\operatorname{um} 1[4,1]), 4])==0, \mathrm{~S}[4]<-\mathrm{NA}, \mathrm{S}[4]<-\mathrm{AA}[$ which ( $\mathrm{AA}[, 5]==\operatorname{um} 1[4,1]), 4]) \quad$ \#captura o valor da variável $\mathrm{S}(\mathrm{u} 4)$

if els e $(\operatorname{length}(\mathrm{AA}[$ which $(\mathrm{AA}[, 5]==\operatorname{um} 1[5,1]), 4])==0, \mathrm{~S}[5]<-\mathrm{NA}, \mathrm{S}[5]<-\mathrm{AA}[\mathrm{which}($ $\mathrm{AA}[, 5]==\operatorname{um} 1[5,1]), 4]) \quad$ \#captura o valor da variável $\mathrm{S}(\mathrm{u} 5)$

ifelse (length $(\mathrm{AA}[$ which $(\mathrm{AA}[, 5]==\operatorname{um} 1[6,1]), 4])==0, \mathrm{~S}[6]<-\mathrm{NA}, \mathrm{S}[6]<-\mathrm{AA}[$ which ( $\mathrm{AA}[, 5]==\operatorname{um} 1[6,1]), 4]) \quad$ \#captura o valor da variável $\mathrm{S}(\mathrm{u} 6)$

ifelse $($ length $(\mathrm{AA}[$ which $(\mathrm{AA}[, 5]==\operatorname{um} 1[7,1]), 4])==0, \mathrm{~S}[7]<-\mathrm{NA}, \mathrm{S}[7]<-\mathrm{AA}[$ which ( $\mathrm{AA}[, 5]==\operatorname{um} 1[7,1]), 4]) \quad$ \#captura o valor da variável $\mathrm{S}(\mathrm{u} 7)$

ifelse (length $(\mathrm{AA}[$ which $(\mathrm{AA}[, 5]==\operatorname{um} 1[8,1]), 4])==0, \mathrm{~S}[8]<-\mathrm{NA}, \mathrm{S}[8]<-\mathrm{AA}[$ which ( $\mathrm{AA}[, 5]==\operatorname{um} 1[8,1]), 4]) \quad$ \#captura o valor da variável $\mathrm{S}(\mathrm{u} 8)$

if els e (length $(\mathrm{AA}[$ which $(\mathrm{AA}[, 5]==\operatorname{um} 1[9,1]), 4])==0, \mathrm{~S}[9]<-\mathrm{NA}, \mathrm{S}[9]<-\mathrm{AA}[$ which ( $\mathrm{AA}[, 5]==\operatorname{um} 1[9,1]), 4]) \quad$ \#captura o valor da variável $\mathrm{S}(\mathrm{u} 9)$

if els e $(\operatorname{length}(\mathrm{AA}[$ which $(\mathrm{AA}[, 5]==\operatorname{um} 1[10,1]), 4])==0, \mathrm{~S}[10]<-\mathrm{NA}, \mathrm{S}[10]<-\mathrm{AA}[$ which $(\mathrm{AA}[, 5]==\operatorname{um} 1[10,1]), 4])$ \#captura o valor da variável $\mathrm{S}(\mathrm{u} 10)$

if else $(\operatorname{length}(\mathrm{AA}[$ which $(\mathrm{AA}[, 5]==\operatorname{um} 1[11,1]), 4])==0, \mathrm{~S}[11]<-\mathrm{NA}, \mathrm{S}[11]<-\mathrm{AA}[$ which $(\mathrm{AA}[, 5]==\operatorname{um} 1[11,1]), 4])$ \#captura o valor da variável $\mathrm{S}(\mathrm{u} 11)$

if els e $(\operatorname{length}(\mathrm{AA}[$ which $(\mathrm{AA}[, 5]==\operatorname{um} 1[12,1]), 4])==0, \mathrm{~S}[12]<-\mathrm{NA}, \mathrm{S}[12]<-\mathrm{AA}[$ which $(\mathrm{AA}[, 5]==\operatorname{um} 1[12,1]), 4]) \quad$ \#captura o valor da variável $\mathrm{S}(\mathrm{u} 12)$

\# incrementar aqui se aumentar os níveis do template

\# modificando desde aqui para probabilidades de todo tipo

Valor_S<-as.vector $(\mathrm{S}[$ which $($ is . na $(\mathrm{S}) !=\mathrm{TRUE})])$ \# O valor do posto $\mathrm{S}$ posto_S $<$-as.vector (which (is . na $(\mathrm{S}) !=$ TRUE) ) \# Posto $\mathrm{S}$

ifelse (length ( posto_S $)>0$, Nivel_prob $<-$ length $($ posto_S $)$, Nivel_prob $<-0)$

ifelse $($ Nivel_prob $==0$, conta $13<-$ conta $13+1,\{\quad \#$ garantindo amostras dentro do template de busca Nível 0 não entra

$\mathrm{X}<-$ matrix $(\mathrm{NA}$, nrow $($ Imagem_treino $)$, Nivel_prob $) \quad$ \# matriz dinâmica

if $($ Nivel_prob $>0)\{$

for ( $\mathrm{i}$ in $1:$ length (posto_s $)$ )

$\{\mathrm{X}[, \mathrm{i}]<-$ Imagem_treino $[, 4 \overline{+}$ posto_S $[\mathrm{i}]]\}$

\} 
390

391

392

393

394

\# $\mathrm{p}_{-} \mathrm{util}<-\mathrm{c}()$

\# calculo das probabilidades condicionais que serão alocadas para a amostra sorteada

if else (Nivel_prob $==1,\{$

prop_1<-data.frame (prop.table (table (Imagem_treino $[, 4], \mathrm{X}[, 1]), 2: 2)$ )

prop_u1<-prop_1[which (is . nan (prop_1 [, "Freq"])!=TRUE) ,]

$p_{-} u t i l<-a s . \operatorname{vector}\left(\operatorname{prop}_{-} u 1\left[\operatorname{which}\left(\operatorname{prop}_{-} u 1[, 2]==\right.\right.\right.$ Valor_S [1] $\left.\left.), 3\right]\right)$

if else $\left(\right.$ length $\left(p_{-}\right.$util $)==0$, conta $1<-\operatorname{conta} 1+1,\{$

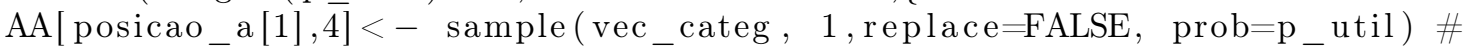
estimativa de novos valores

$\mathrm{AA}\left[\right.$ posicao_a[1],21] $<-\mathrm{p}_{\text {_util }}[1]$ \# a probabilidade não condicional utilizada para estimativa da categoria 1: Level

AA[posicao_a[1],22]<- p_util[2] \# a probabilidade não condicional utilizada para estimativa da categoria 2: Level

AA[posicao_a[1],23]<- p_util[3] \# a probabilidade não condicional utilizada para estimativa da categoria 3: Level

AA[posicao_a[1],24]<- p_util[1]*(1- p_util[1]) \# a incerteza relacionada a categoria 1: Level

$\mathrm{AA}\left[\right.$ posicao_a[1],25]<- _ $_{-}$util [2]*(1- _ $_{-}$util [2] $)$\# a incerteza relacionada a categoria 2: Level

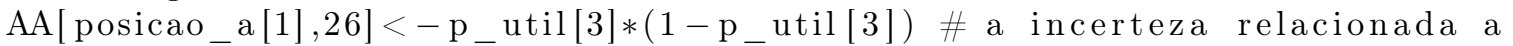
categoria 3: Level

$\mathrm{AA}[$ posicao_a[1],6]<-Nivel_prob \# Level

AA[posicao_a[1],7]<-Valor_S [1]

$\mathrm{AA}[$ posicao_a[1],31]<- posto_S [1]

posicao_a $<-$ posicao_a $[-1]$

\}

\}

, ifelse (Nivel_prob $==2,\{$

prop_1<-data.frame (prop.table (table (Imagem_treino [,4],X[,1]),2:2))

prop_u $1<-$ prop_$_{-} 1$ [which $($ is . nan (prop_1 [, "Freq"] $) !=$ TRUE) ,]

p_util1<-as.vector $\left(\operatorname{prop} \_u 1\left[\operatorname{which}\left(\overline{p r o p}_{-} u 1[, 2]==\right.\right.\right.$ Valor_S $\left.\left.\left.[1]\right), 3\right]\right)$

prop_2<-data.frame (prop.table (table (Imagem_treino [,4],X[,1],X[,2]) ,2:3)) prop_u $2<-$ prop_2 [which (is . nan (prop_2 [, "Freq"] $) !=$ TRUE) ,]

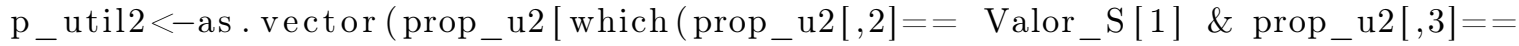
Valor_S [2]),4])

ifelse (length $\left(p_{-}\right.$util2 $) !=0, p_{-}$util $<-p_{-}$util2, ifelse $\left(\right.$length $\left(p_{-}\right.$util1 $) !=0$, $\left.\left.\mathrm{p}_{\text {_util }}<-\mathrm{p}_{-} \mathrm{util} 1, \mathrm{p}_{\text {_ }} \mathrm{util}<-\mathrm{c}()\right)\right)$

if else $\left(\right.$ length $\left(p_{-}\right.$util $)==0, \quad$ conta $2<-\operatorname{conta} 2+1,\{$

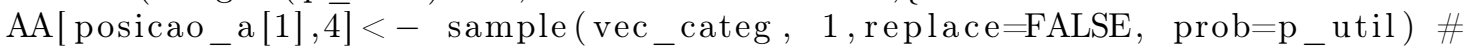
estimativa de novos valores

$\mathrm{AA}[$ posicao_a[1],21]<- p_util[1] \# a probabilidade não condicional utilizada para estimativa da categoria 1: Level 
410 AA[posicao_a[1],22]<-p_util[2] \# a probabilidade não condicional utilizada para estimativa da categoria 2: Level

411 AA[posicao_a[1],23]<-p_util[3] \# a probabilidade não condicional utilizada para estimativa da categoria 3: Level

412 AA[posicao_a[1],24]<-p_util[1]*(1-p_util[1]) \# a incerteza relacionada a categoria 1: Level

413 AA[posicao_a[1],25]<-p_util[2]*(1-p_util[2]) \# a incerteza relacionada a categoria 2: Level

414 AA[posicao_a[1],26]<- p_util[3]*(1-p_util[3]) \# a incerteza relacionada a categoria 3: Level

415 AA[ posicao_a[1],6]<-Nivel_prob \# Level

416 AA[ posicao_a [1],7]<-Valor_S [1]

417 AA[posicao_a $[1], 8]<-$ Valor_S $[2]$

418 AA[posicao_a $[1], 31]<-$ posto_S $[1]$

419 AA[posicao_a $[1], 32]<-$ posto_S $[2]$

420 posicao_a $<-$ posicao_a $[-1]$

$421\}$

422 )

423

424

425

426

427

428

429

430

431

432

433

434

435

436

437

438

, if else (Nivel_prob $==3,\{$

prop_1<-data.frame (prop.table (table (Imagem_treino [,4], X [, 1]),2:2))

prop_u1<-prop_1[which(is .nan (prop_1[, "Freq"] $) !=$ TRUE), ]

$p_{-} u t i 11<-a s . v e c t o r\left(p_{1} p_{-} u 1\left[w h i c h\left(\operatorname{prop}_{-} u 1[, 2]==\right.\right.\right.$ Valor_S $\left.\left.\left.[1]\right), 3\right]\right)$

prop_2<-data.frame (prop.table(table (Imagem_treino [,4],X[,1],X[,2] ), 2:3))

prop_u $2<-$ prop_2[which(is .nan(prop_2[, "Freq"] $) !=$ TRUE), ]

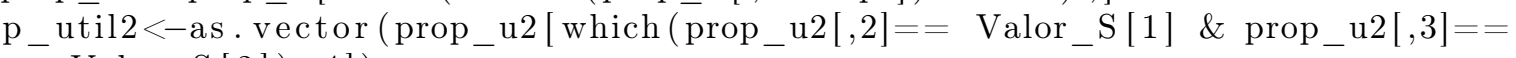
Valor_S [2]),4])

prop_3<-data.frame (prop.table (table (Imagem_treino [ , 4] , X [ , 1] , X [ , 2] , X [ , 3]) $, 2: 4))$

prop_u3<-prop_3[which (is .nan (prop_3 [, "Freq"])!=TRUE) ,]

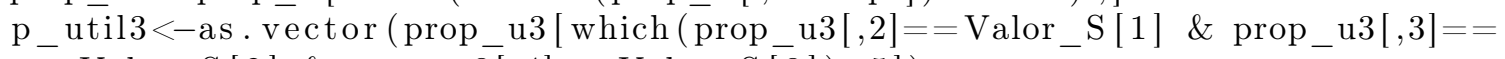
Valor_S [2] \& prop_u3[,4]==Valor_S $[3]), 5])$

442

ifelse (length $\left(p_{-}\right.$util3 $) !=0, p_{-} u t i l<-p_{-} u t i l 3$, ifelse $\left(\right.$ length $\left(p_{-}\right.$util2 $) !=0$, $p_{-} u t i l<-p_{-} u t \overline{t i} 2$, ifelse $\left(\right.$ length $\left(p_{-}\right.$util1 $) !=0, p_{-} u t i l<-p_{-} u t i l 1, p_{-} u t i l<-c$ ()$\left.\left.^{-}\right)\right)$

443

444 ifelse $\left(\right.$ length $\left(p_{-}\right.$util $)=0$, conta $3<-\operatorname{conta} 3+1,\{$

445 AA[posicao_a $[1], 4]<-$ sample $\left(\right.$ vec_categ, $_{-}$, replace=FALSE, prob=p_util) \# estimativa de novos valores

446 AA[posicao_a[1],21]<- p_util[1] \# a probabilidade não condicional utilizada para estimativa da categoria 1: Level

447 AA[posicao_a[1],22]<- p_util[2] \# a probabilidade não condicional utilizada para estimativa da categoria 2: Level

448 AA[posicao_a[1],23]<- p_util[3] \# a probabilidade não condicional utilizada para estimativa da categoria 3: Level

449 AA[posicao_a[1],24]<- _ $_{-}$util[1]*(1- p_util[1]) \# a incerteza relacionada a categoria 1: Level

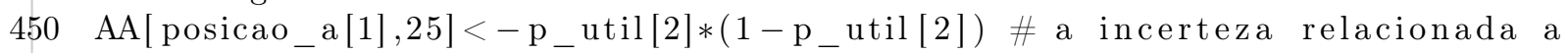
categoria 2: Level

451 AA[posicao_a[1],26]<- _ $_{-}$util [3]*(1- p_util[3]) \# a incerteza relacionada a categoria 3: Level 
461

462 )

463

464

465

466

467

468

469

470

471

472

473

474

475

476

477

478

479

480

481

482

483

484

485

486

487

488

489

490 ifelse $\left(\right.$ length $\left(p_{-}\right.$util $)==0, \quad$ conta $4<-$ conta $4+1,\{$

491

492

493

494

495

496

$\mathrm{AA}[$ posicao_a[1],6]<-Nivel_prob \# Level

$\mathrm{AA}[$ posicao_a [1],7]<-Valor_S [1]

$\mathrm{AA}[$ posicao_a[1],8]<-Valor_S $[2]$

$\mathrm{AA}[$ posicao_a[1],9]<-Valor_S [3]

$\mathrm{AA}[$ posicao_a[1],31]<-posto_S [1]

$\mathrm{AA}[$ posicao_a [1], 32$]<-$ posto_S [2]

$\mathrm{AA}[$ posicao_a[1],33]<-posto_S [3]

posicao_a $<-$ posicao_a $[-1]$

\}

\}

, ifelse (Nivel_prob $==4,\{$

Valor_S [2]),4]) $, 2: 4))$ $[, 4]), 2: 5))$ estimativa de novos valores categoria 1: Level prop_1<-data.frame (prop.table (table (Imagem_treino [,4],X[,1]),2:2))

prop_u $1<-\operatorname{prop}_{-} 1\left[\right.$ which $\left(\right.$ is . nan $\left(\operatorname{prop}_{-} 1[\right.$, "Freq"] $) !=$ TRUE) ,]

p_util1<-as.vector $\left(\operatorname{prop} \_u 1\left[\operatorname{which}\left(\overline{p r o p}_{-} u 1[, 2]==\right.\right.\right.$ Valor_S $\left.\left.\left.[1]\right), 3\right]\right)$

prop_2<-data.frame (prop.table (table (Imagem_treino [,4],X[,1],X[,2]),2:3))

prop_u $2<-$ prop_2[which (is . nan (prop_2 [, "Freq"] )!=TRUE) ,]

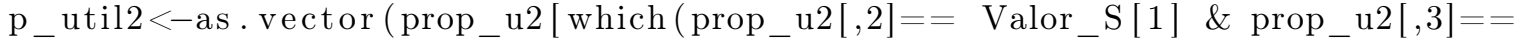

prop_3<-data.frame (prop.table (table (Imagem_treino [,4],X[,1],X[,2],X[,3])

prop_u $3<-$ prop_3 [which (is . nan (prop_3 [, "Freq"])!=TRUE) ,]

p_util3<-as.vector (prop_u3 [which (prop_u3[,2]==Valor_S [1] \& prop_u3[,3]==

Valor_S [2] \& prop_u3[, 4]==Valor_S $[3]), 5])$

prop_4<-data.frame (prop.table (table (Imagem_treino [,4],X[, 1], X[, 2] ,X [, 3], X

prop_u $4<-$ prop_4 [which ( is . nan (prop_4 [, "Freq"])!=TRUE) ,]

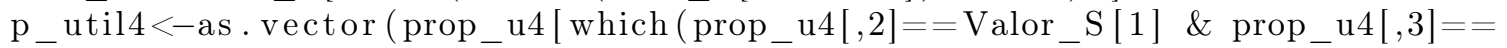

Valor_S [2] \& prop_u4[,4]==Valor_S [3] \& prop_u4[, $\overline{5}]==$ Valor_S $[4]), 6])$

ifelse (length $\left(p_{-}\right.$util4 $) !=0, p_{-}$util $<-p_{-}$util4, ifelse $\left(\right.$length $\left(p_{-}\right.$util 3$) !=0$,

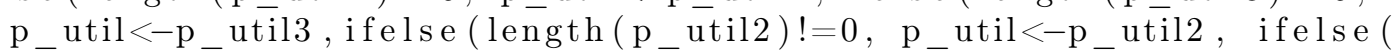
length $\left(p_{-}\right.$utill $\left.\left.\left.) !=0, p_{-} u t i l<-p_{-} u t i l 1, p_{-} u t i l<-\bar{c}() \quad\right)\right)\right)$

$\mathrm{AA}\left[\right.$ posicao_a[1],4]<- sample $_{\text {(vec_categ, }}$ 1, replace=FALSE, prob=p_util) \#

$\mathrm{AA}[$ posicao_a[1],21]<-p_util[1] \# a probabilidade não condicional utilizada para estimativa da categoria 1: Level

$\mathrm{AA}[$ posicao_a[1],22]<-p_util[2] \# a probabilidade não condicional utilizada para estimativa da categoria 2: Level

AA[posicao_a[1],23]<-p_util[3] \# a probabilidade não condicional utilizada para estimativa da categoria 3: Level

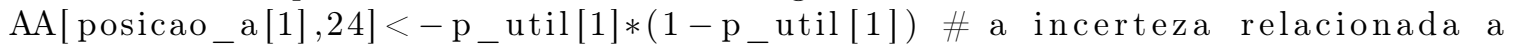

$\mathrm{AA}\left[\right.$ posicao_a[1],25]<- _ $_{-}$util[2]*(1-p_util[2]) \# a incerteza relacionada a categoria 2: Level 
49

498

499

500

501

502

503

504

505

506

507

508

509

510 )

511

512

513

514

515

516

517

518

519

520

521

522

523

524

525

526

527

528

529

530

531

532

533

534

535

536

537

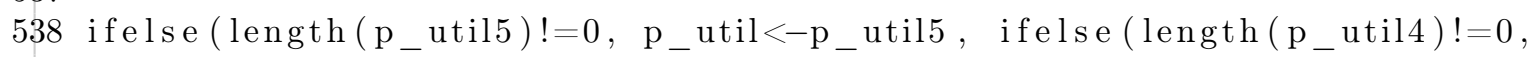
$p_{-}$util $<-p_{-}$util4, if else $\left(\operatorname{length}\left(p_{-} \overline{u t i l}_{3}\right) !=0, p_{-}\right.$util $<-p_{-} u t i \bar{l} 3$, ifelse ( length $\left(p_{-}\right.$util2 $) !=0, p_{-} u t i l<-p_{-} u t i l 2, \quad$ ifelse $\left(\overline{l e n g t h}\left(p_{-} u t i l 1\right) !=0, p_{-}\right.$util 539

540

541

542 ifelse $\left(\operatorname{length}\left(p_{-}\right.\right.$util $)==0, \operatorname{conta} 5<-\operatorname{conta} 5+1,\{$

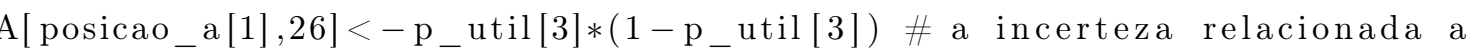
categoria 3: Level

$\mathrm{AA}[$ posicao_a[1],6]<-Nivel_prob \# Level

$\mathrm{AA}[$ posicao_a [1],7]<-Valor_S [1]

$\mathrm{AA}[$ posicao_a $[1], 8]<-$ Valor_S $[2]$

$\mathrm{AA}[$ posicao_a[1],9]<-Valor_S [3]

$\mathrm{AA}[$ posicao_a[1],10]<-Valor_S [4]

$\mathrm{AA}[$ posicao_a[1],31]<-posto_S $[1]$

$\mathrm{AA}[$ posicao_a [1],32]<- posto_S [2]

$\mathrm{AA}[$ posicao_a [1],33]<-posto_S $[3]$

$\mathrm{AA}[$ posicao_a [1],34]<- posto_S [4]

posicao_a $<-$ posicao_a $[-1]$

\}

\}

, ifelse (Nivel_prob $==5,\{$

prop_1<-data.frame (prop.table (table (Imagem_treino $[, 4], \mathrm{X}[, 1]), 2: 2))$

prop_u $1<-$ prop_1 $_{\text {[ which }}\left(\right.$ is . nan $\left(\operatorname{prop}_{-} 1[\right.$, "Freq"] $) !=$ TRUE),$]$

p_util1<-as.vector $\left(\operatorname{prop} \_u 1\left[\operatorname{which}\left(\operatorname{prop}_{-} u 1[, 2]==\right.\right.\right.$ Valor_S [1] $\left.\left.), 3\right]\right)$

prop_2<-data.frame (prop.table (table (Imagem_treino [,4],X[,1],X[,2]),2:3))

prop_u $2<-$ prop_2 [which (is . nan (prop_2 [, "Freq"] )!=TRUE), ]

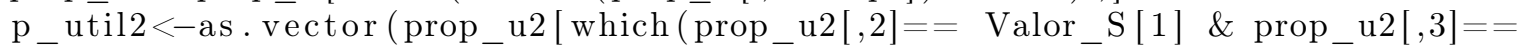
Valor_S [2]),4])

prop_3<-data.frame (prop.table (table (Imagem_treino [,4],X[,1],X[,2],X[, 3]) $, 2: 4))$

prop_u $3<-$ prop_3 [which ( is . nan (prop_3 [, "Freq"])!=TRUE) ,]

p_util3<-as.vector (prop_u3 [which ( Valor_S [2] \& prop_u3[,4]==Valor_S [3]), 5])

prop_4<-data.frame (prop.table (table (Imagem_treino [, 4], X[, 1], X [ , 2] , X [, 3], X $[, 4]), 2: 5))$

prop_u4<-prop_4 [which (is . nan (prop_4 [, "Freq"])!=TRUE) ,]

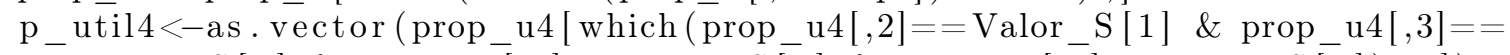

Valor_S [2] \& prop_u4[,4]==Valor_S [3] \& prop_u4[, $\overline{5}]==$ Valor_S $[4]), 6])$

prop_5<-data.frame (prop.table (table (Imagem_treino [,4],X[, 1], X [ , 2], X [, 3], X $[, 4], \mathrm{X}[, 5]), 2: 6))$

prop_u $5<-$ prop_5[which (is . nan (prop_5 [, "Freq"])!=TRUE),]

$p_{\_}$util $5<-$ as.vector $\left(\operatorname{prop}_{-}\right.$u $5\left[\right.$ which $\left(\operatorname{prop}_{-} \mathrm{u} 5[, 2]==\right.$ Valor_S $[1] \&$ prop_u $5[, 3]==$ Valor_S [2] \& prop_u $\overline{\mathrm{u}}[, 4]==$ Valor_S $_{3}[3] \&$ prop_u$_{-}[, 5]==$ Valor_S $[4] \&$ prop_u $_{-} \overline{5}[, 6]==$ Valor_S $\left.\left.\left.[5]\right), 7\right]\right)$ $<-p_{-}$util1, p_util<-c() $\left.\left.\left.)\right)\right)\right)$) 
543 AA[posicao_a[1],4]<- sample(vec_categ, 1, replace=FALSE, prob=p_util) \# estimativa de novos valores

544 AA[posicao_a[1],21]<- p_util[1] \# a probabilidade não condicional utilizada para estimativa da categoria 1: Level

545 AA[posicao_a[1],22]<- p_util[2] \# a probabilidade não condicional utilizada para estimativa da categoria 2: Level

546 AA[posicao_a[1],23]<-p_util[3] \# a probabilidade não condicional utilizada para estimativa da categoria 3: Level

547 AA[posicao_a[1],24]<- _ $_{-}$util[1]*(1- p_util[1]) \# a incerteza relacionada a categoria 1: Level

548 AA[posicao_a[1],25]<- p_util[2]*(1- p_util[2]) \# a incerteza relacionada a categoria 2: Level

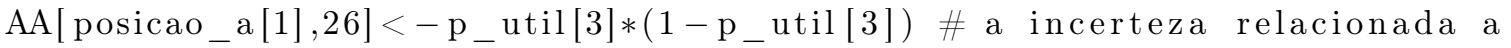
categoria 3: Level

prop_4<-data.frame (prop.table (table (Imagem_treino [, 4], X[, 1], X [ , 2] , X [ , 3], X $[, 4]), 2: 5))$

prop_u $4<-$ prop_4 [which ( is . nan (prop_4 [, "Freq"])!=TRUE) ,]

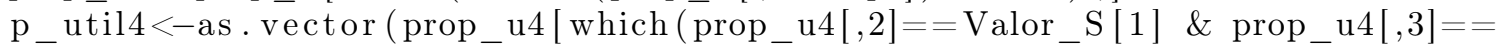
Valor_S [2] \& prop_u4[,4]==Valor_S $[3] \&$ prop_u$_{-}[\overline{, 5}]==$ Valor_S $\left.\left.\left.[4]\right), 6\right]\right)$ 
590

591

601

602

prop_5<-data.frame (prop.table (table (Imagem_treino [ , 4], X [ , 1], X $[, 2], \mathrm{X}[, 3], \mathrm{X}$ $[, 4], \mathrm{X}[, 5]), 2: 6))$ prop_u $5<-$ prop_5[which(is .nan(prop_5[, "Freq" ])!=TRUE), ] p_util5<-as.vector (prop_u5[which(prop_u5 $[, 2]==$ Valor_S $[1] \&$ prop_u5 $[, 3]==$ Valor_S[2] \& prop_u5[,4]==Valor_S[3] \& prop_u5[,5]==Valor_S[4] \& prop_us $[, 6]==$ Valor_S $[5]), 7]$ )

prop_6<-data.frame (prop.table(table (Imagem_treino [ , 4] , X [ , 1] , X [ , 2], X [ , 3] , X $[, 4], \mathrm{X}[, 5], \mathrm{X}[, 6]), 2: 7))$

prop_u6<-prop_6[which(is .nan(prop_6[, "Freq"])!=TRUE), ]

p_util6<-as.vector (prop_u6[which (prop_u6[,2]==Valor_S [1] \& prop_u6[,3]== Valor_S[2] \& prop_u6[,4]==Valor_S[3] \& prop_u6[,5]== Valor_S[4] \& prop_u6[,6]==Valor_S[5] \& prop_u6[,7]==Valor_S[6]),8])

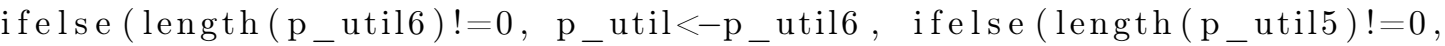

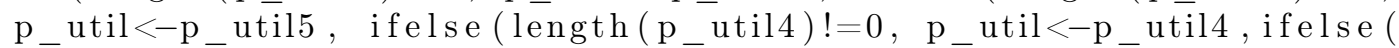
length $\left(p_{-}\right.$util3 $) !=0, p_{-}$util $<-p_{-}$util3, ifelse $\left(\right.$length $\left(p_{-}\right.$util 2$) !=0, p_{-}$util $<-$ $p_{-}$util2, ifelse $\left.\left.\left.\left(\operatorname{length}\left(p_{-} u t i l \overline{1}\right) !=0, p_{-} u t i l<-p_{-} u t i l 1, p_{-} u t i l<-c() \overline{)}\right)\right)\right)\right)$ )

ifelse $\left(\right.$ length $\left(p_{-}\right.$util $)==0, \operatorname{conta} 6<-\operatorname{conta} 6+1,\{$

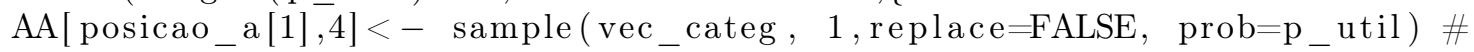
estimativa de novos valores

AA[posicao_a[1],21]<- p_util[1] \# a probabilidade não condicional utilizada para estimativa da categoria 1: Level

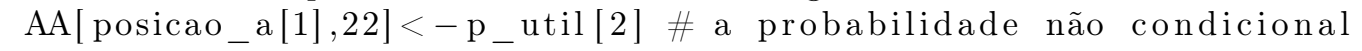
utilizada para estimativa da categoria 2: Level

$\mathrm{AA}[$ posicao_a[1],23]<- p_util[3] \# a probabilidade não condicional utilizada para estimativa da categoria 3: Level

AA[posicao_a [1],24] $<-p_{-}$util $[1] *\left(1-p_{-}\right.$util [1]) \# a incerteza relacionada a categoria 1: Level

$\mathrm{AA}[$ posicao_a $[1], 25]<-\mathrm{p}_{-} \mathrm{util}[2] *\left(1-\mathrm{p}_{-}\right.$util[2] $) \#$ a incerteza relacionada a categoria 2: Level

$\mathrm{AA}[$ posicao_a $[1], 26]<-p_{\text {_ }}$ util $[3] *\left(1-\mathrm{p}_{\text {_ }}\right.$ util[3] $)$ \# a incerteza relacionada a categoria 3: Level

AA[posicao_a [1],6]<-Nivel_prob \# Level

$\mathrm{AA}[$ posicao_a $[1], 7]<-$ Valor_S $[1]$

$\mathrm{AA}[$ posicao_a[1],8]<-Valor_S $[2]$

AA[posicao_a[1],9]<-Valor_S $[3]$

AA [posicao_a [1],10]<-Valor_S $[4]$

AA [ posicao_a [1],11]<-Valor_S [5]

AA [ posicao_a [1],12]<-Valor_S [6]

$\mathrm{AA}[$ posicao_a [1],31]<-posto_S [1]

AA [posicao_a [1],32]<-posto_S [2]

AA [ posicao_a [1],33]<-posto_S [3]

AA [ posicao_a [1],34]<-posto_S $[4]$

AA[posicao_a $[1], 35]<-$ posto_S $[5]$

$\mathrm{AA}[$ posicao_a $[1], 36]<-$ posto_S $[6]$

posicao_a $<-$ posicao_a $[-1]$

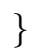


632

633

634

635

636

637

638

639

640

641

642

643

644

645

646

647

648

649

650

651

652

653

654

655

656

657

658

659

660

661

662

663

664

665

666

667 prop_u $1<-$ prop_$_{-} 1\left[\right.$ which $\left(\right.$ is . nan $\left(\operatorname{prop}_{-} 1[\right.$, "Freq" ])!=TRUE) ,]

p_util1<-as . vector $\left(\operatorname{prop}_{-} u 1\left[\right.\right.$ which $\left(\operatorname{prop}_{-} u 1[, 2]==\right.$ Valor_S [1] $\left.\left.), 3\right]\right)$

prop_2<-data.frame (prop.table (table (Imagem_treino [,4],X[,1],X[,2]),2:3)) prop_u $2<-$ prop_2[which (is .nan (prop_2[, "Freq"] )!=TRUE) ,]

p_util $2<-$ as . vector $\left(\operatorname{prop} \_u 2\left[\right.\right.$ which $\left(\operatorname{prop}_{-} u 2[, 2]==\right.$ Valor_S $[1]$ \& prop_u2[,3]== Valor_S [2]),4])

prop_3<-data.frame (prop.table (table (Imagem_treino [, 4], X[, 1], X[, 2] , X [ , 3]) ,2:4))

prop_u $3<-$ prop_3 [which ( is .nan (prop_3 [, "Freq"])!=TRUE) ,]

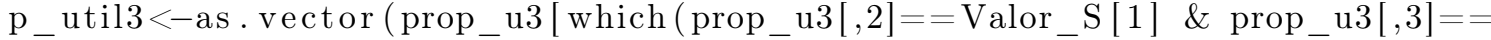

Valor_S [2] \& prop_u3[,4]==Valor_S $[3]), 5])$

prop_4<-data.frame (prop.table (table (Imagem_treino [,4],X[, 1], X [ ,2], X [, 3], X $[, 4]), 2: 5))$

prop_u $4<-$ prop_4 [which ( is .nan $\left(\operatorname{prop}_{-} 4[\right.$, "Freq" $\left.]\right) !=$ TRUE $\left.),\right]$

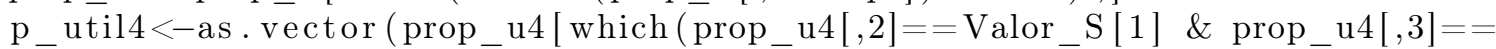

Valor_S [2] \& prop_u4[,4]==Valor_S [3] \& prop_u4[, 5$]==$ Valor_S $[4]), 6])$

prop_5<-data.frame (prop.table (table (Imagem_treino [,4],X[, 1], X [ , 2] ,X [, 3], X $[, 4], \mathrm{X}[, 5]), 2: 6))$

prop_u $5<-$ prop_5[which ( is . nan (prop_5 [, "Freq"])!=TRUE) ,]

p_util5<-as.vector (prop_u5[which(prop_u5[,2]== Valor_S [1] \& prop_u5[,3]==

Valor_S [2] \& prop_u5[,4]==Valor_S [3] \& prop_u5[,5]==Valor_S [4] \& prop_u $\overline{5}[, 6]==$ Valor_S $[5]), 7])$

prop_6<-data.frame (prop.table (table (Imagem_treino [, 4], X[ , 1], X [ , 2] , X [ , 3], X $[, 4], \mathrm{X}[, 5], \mathrm{X}[, 6]), 2: 7))$

prop_u6<-prop_6[which (is .nan (prop_6 [, "Freq"])!=TRUE) ,]

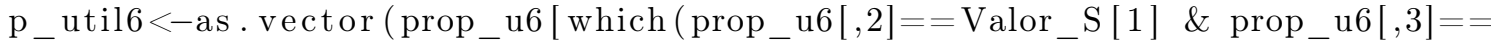

Valor_S [2] \& prop_u6[,4]==Valor_S [3] \& prop_u6[, 5$]==$ Valor_S $[4] \&$

prop_u6[,6]==Valor_S[5] \& prop_u6[,7]==Valor_S[6]) ,8])

prop_ $7<$-data.frame (prop.table (table (Imagem_treino [, 4],X[, 1], X[ , 2], X [, 3], X $[, 4], \mathrm{X}[, 5], \mathrm{X}[, 6], \mathrm{X}[, 7]), 2: 8))$

prop_u $7<-$ prop_7 [which (is . nan (prop_7 [, "Freq"])!=TRUE) ,]

p_util7<-as.vector (prop_u7 [which(prop_u7 [,2]== Valor_S [1] \& prop_u7 [,3]==

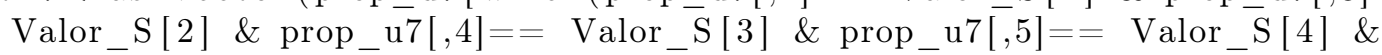

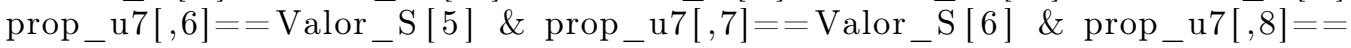
Valor_S [7]),9])

ifelse $\left(\operatorname{length}\left(p_{-}\right.\right.$util 7$) !=0, p_{\_}$util $<-p_{-}$util7, ifelse $\left(\operatorname{length}\left(p_{-}\right.\right.$util6 $) !=0$, $p_{-}$util $<-p_{-}$util6, ifelse $\left(\operatorname{length}\left(p_{-}\right.\right.$util5 $) !=0, p_{-}$util $<-p_{-}$utils, ifelse (

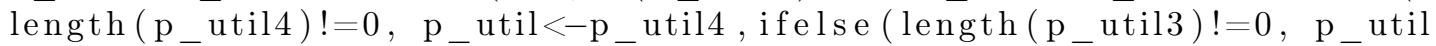

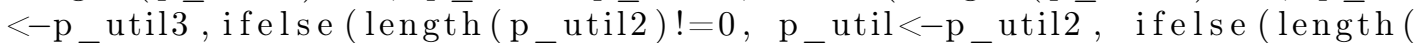
$\left.\left.\left.\left.\left.\left.\left.p_{-} u t i l 1\right) !=0, p_{-} u t i l<-p_{-} u t \overline{i l} 1, p_{-} u t i l<-c \overline{(}\right)()\right)\right)\right)\right)\right)$

if else $\left(\operatorname{length}\left(p_{-}\right.\right.$util $)==0$, conta $7<-\operatorname{conta} 7+1,\{$

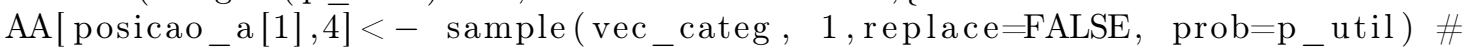
estimativa de novos valores

$\mathrm{AA}[$ posicao_a[1],21]<-p_util[1] \# a probabilidade não condicional utilizada para estimativa da categoria 1: Level

$\mathrm{AA}[$ posicao_a[1],22]<- p_util[2] \# a probabilidade não condicional utilizada para estimativa da categoria 2: Level 
668

669

691

692

693

694

695

696

697

698

699

700

701

702

703

AA[posicao_a[1],23]<- p_util[3] \# a probabilidade não condicional utilizada para estimativa da categoria 3: Level

AA[posicao_a[1],24]<- p_util[1]*(1- p_util[1]) \# a incerteza relacionada a categoria 1: Level

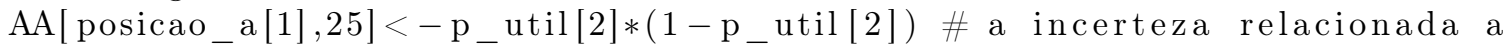
categoria 2: Level

$\mathrm{AA}[$ posicao_a[1],26]<-p_util[3]*(1-p_util[3]) \# a incerteza relacionada a categoria 3: Level

AA posicao a $[1], 6]<-$ Nivel prob \# Level

$\mathrm{AA}[$ posicao_a [1],7]<-Valor_S [1]

$\mathrm{AA}[$ posicao_a $[1], 8]<-$ Valor_S $[2]$

AA[posicao_a[1],9]<-Valor_S [3]

$\mathrm{AA}[$ posicao_a[1],10]<-Valor_S [4]

$\mathrm{AA}[$ posicao_a [1],11]<-Valor_S [5]

AA [ posicao_a[1],12]<-Valor_S [6]

AA [ posicao_a[1],13]<-Valor_S [7]

$\mathrm{AA}[$ posicao_a[1],31]<-posto_S [1]

$\mathrm{AA}[$ posicao_a[1],32]<-posto_S [2]

AA[posicao_a[1],33]<-posto_S [3]

$\mathrm{AA}[$ posicao_a [1],34]<- posto_S [4]

$\mathrm{AA}[$ posicao_a[1],35]<-posto_S [5]

$\mathrm{AA}[$ posicao_a[1],36]<-posto_S $[6]$

$\mathrm{AA}[$ posicao_a[1],37]<-posto_S [7]

posicao_a $<-$ posicao_a $[-1]$

prop_4<-data.frame (prop.table (table (Imagem_treino [,4],X[, 1], X [ , 2] , X [ , 3], X $[, 4]), 2: 5))$

prop_u4<-prop_4 [which (is . nan (prop_4 [, "Freq"])!=TRUE) ,]

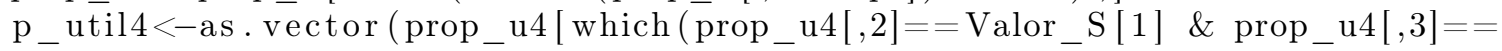

Valor_S [2] \& prop_u4[,4]==Valor_S [3] \& prop_u4[, 5$]==$ Valor_S $[4]), 6])$

prop_5<-data.frame (prop.table (table (Imagem_treino [,4],X[, 1], X [ , 2] , X [, 3], X $[, 4], \mathrm{X}[, 5]), 2: 6))$

prop_u $5<-$ prop_$_{-}\left[\right.$which $\left(\right.$is . nan $\left(\operatorname{prop}_{-} 5[\right.$, "Freq" $\left.]\right) !=$ TRUE) ,]

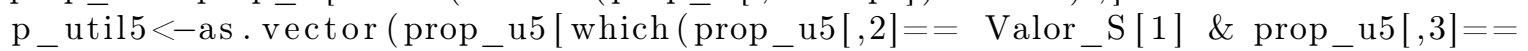
Valor_S [2] \& prop_u5[,4]== Valor_S $[3] \& \operatorname{prop}_{-} \mathrm{u} 5[, 5]==$ Valor_S $[4] \&$ 


$$
\text { prop_u5[,6]==Valor_S [5]),7]) }
$$

prop_6<-data.frame(prop.table(table(Imagem_treino [ , 4] , X [ , 1] , X [ ,2], X [ , 3], X $[, 4], \mathrm{X}[, 5], \mathrm{X}[, 6]), 2: 7))$

prop_u6<-prop_6[which(is .nan (prop_6[, "Freq"])!=TRUE) ,]

p_util6<-as .vector (prop_u6[which (prop_u6[,2]==Valor_S[1] \& prop_u6[,3]==

Valor_S[2] \& prop_u6[,4]==Valor_S $[3]$ \& prop_u6[, 5$]==$ Valor_S $[4] \&$ prop_u6 $[, 6]==$ Valor_S $[5] \&$ prop_u6 $[, 7]==$ Valor_S [6] $), 8])$

prop_ $7<$-data.frame(prop.table (table(Imagem_treino [ , 4] , X [ , 1] , X [ , 2] , X [ , 3] , X $[, 4], \mathrm{X}[, 5], \mathrm{X}[, 6], \mathrm{X}[, 7]), 2: 8))$

prop_u7<-prop_7[which(is .nan (prop_7 [, "Freq"])!=TRUE) ,] $p_{-}$util7<-as.vector $($prop_u7 [which (prop_u7[,2] = = Valor_S[1] \& prop_u7[,3]== Valor_S[2] \& prop_u7[,4]==Valor_S [3] \& prop_u7[,5]== Valor_S $[4] \&$ prop_u7 $[, 6]==$ Valor_S $[5] \&$ prop_u $\overline{7}, 7]==$ Valor_S $[6] \&$ prop_u7 $[\overline{, 8}]==$ Valor_S [7]),9])

prop_8<-data.frame(prop.table (table(Imagem_treino [ , 4] , X [ , 1], X [ ,2], X [ , 3], X $[, 4], \mathrm{X}[, 5], \mathrm{X}[, 6], \mathrm{X}[, 7], \mathrm{X}[, 8]), 2: 9))$

prop_u $8<-$ prop_8[which (is .nan(prop_8[, "Freq"])!=TRUE) ,]

p_util8<-as.vector (prop_u8[which (prop_u8[,2]==Valor_S[1] \& prop_u8[,3]== Valor_S [2] \& prop_u8[,4]==Valor_S [3] \& prop_u8[, 5$]==$ Valor_S $[4] \&$ prop_u $8[, 6]==$ Valor_S $[5] \&$ prop_u $8[, 7]==$ Valor_S $[6] \&$ prop_u $8[, 8]==$ Valor_S $[7] \&$ prop_u8[,9]==Valor_S $[8]), 10])$

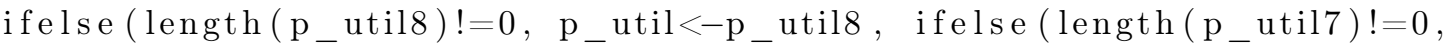
$p_{-}$util $<-p_{-}$util7, ifelse $\left(\right.$length $\left(p_{-}\right.$util6 $) !=0, p_{-}$util $<-p_{-}$util6, ifelse ( length $\left(p_{-}\right.$util5 $) !=0, p_{-}$util $<-p_{-}$util5, if else $\left(\right.$length $\left(p_{-}\right.$util4 $) !=0, p_{-}$util $<-p_{-}$utilu, ifelse $\left(\right.$length $\left(p_{-}\right.$util3 $) !=0, p_{-}$util $<-p_{-}$util3, ifelse (length ( p_util2)!=0, p_util $<-p_{-}$util2, ifelse $\left(\right.$length $\left(p_{-}\right.$utill $) !=0, p_{-}$util $<-$

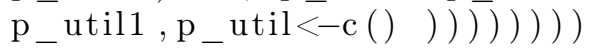

ifelse $\left(\right.$ length $\left(p_{-}\right.$util $)==0$, conta $8<-\operatorname{conta} 8+1,\{$

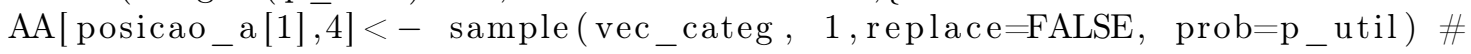
estimativa de novos valores

AA[posicao_a[1],21]<-p_util[1] \# a probabilidade não condicional utilizada para estimativa da categoria 1: Level

$\mathrm{AA}\left[\right.$ posicao_a[1],22]<- ${ }_{\text {_util[2] }}$ \# a probabilidade não condicional utilizada para estimativa da categoria 2: Level

AA[posicao_a[1],23]<- p_util[3] \# a probabilidade não condicional utilizada para estimativa da categoria 3: Level

$\mathrm{AA}\left[\right.$ posicao_a[1],24] $<-\mathrm{p}_{\text {_ }}$ util $[1] *\left(1-\mathrm{p}_{-}\right.$util[1] $) \quad \#$ a incerteza relacionada a categoria 1: Level

AA[posicao_a[1],25]<- p_util[2]*(1-p_util[2]) \# a incerteza relacionada a categoria 2: Level

AA[posicao_a[1],26]<-p_util[3]*(1-p_util[3]) \# a incerteza relacionada a categoria 3: Level

AA $[$ posicao_a $[1], 6]<-$ Nivel_prob \# Level

$\mathrm{AA}[$ posicao_a $[1], 7]<-$ Valor_S $[1]$

AA $[$ posicao_a $[1], 8]<-$ Valor_S $[2]$

AA $[$ posicao_a $[1], 9]<-$ Valor_S $[3]$

AA [ posicao_a [1],10]<-Valor_S [4]

$\mathrm{AA}[$ posicao_a $[1], 11]<-$ Valor_S $[5]$

$\mathrm{AA}[$ posicao_a $[1], 12]<-$ Valor_S $[6]$

AA [ posicao_a [1],13]<-Valor_S [7]

AA [ posicao_a [1],14]<-Valor_S [8]

$\mathrm{AA}[$ posicao_a $[1], 31]<-$ posto_S $[1]$ 
751 AA[posicao_a [1],32] $<-$ posto_S [2]

752 AA[posicao_a[1],33]<-posto_S [3]

753 AA[posicao_a $[1], 34]<-$ posto_S $[4]$

754 AA[posicao_a $[1], 35]<-$ posto_S [5]

755 AA[posicao_a $[1], 36]<-$ posto_S $[6]$

756 AA[posicao_a $[1], 37]<-$ posto_S $[7]$

757 AA[posicao_a $[1], 38]<-$ posto_S $[8]$

758

759

$760\}$

$761)$

762

763

764

765

766

767

768

769

$$
\text { , ifelse (Nivel_prob }==9,\{
$$

prop_1<-data.frame (prop.table (table (Imagem_treino [,4],X[,1]),2:2)) prop_u1<-prop_1[which (is .nan (prop_1[, "Freq"])!=TRUE), ] p_util1<-as.vector $\left(\right.$ prop_u1[which $\left(\operatorname{prop}_{-}\right.$u1[,2]== Valor_S[1]),3])

prop_2<-data.frame (prop.table (table (Imagem_treino[,4],X[,1],X[,2] ),2:3)) prop_u2<-prop_2[which(is .nan(prop_2[, "Freq"] $) !=$ TRUE), ]

p_util2<-as.vector $\left(\operatorname{prop}_{-} u 2\left[\right.\right.$ which $\left(\operatorname{prop}_{-} u 2[, 2]==\right.$ Valor_S [1] \& prop_u2[,3]== Valor_S $[2]), 4])$

prop_3<-data.frame (prop.table (table (Imagem_treino [ ,4] , X [ , 1], X [ ,2] , X [ , 3]) $, 2: 4))$

prop_u3<-prop_3[which(is .nan(prop_3 [, "Freq"])!=TRUE), ]

p_util3<-as.vector $($ prop_u3 [which (prop_u3[,2]==Valor_S[1] \& prop_u3[,3]== Valor_S[2] \& prop_u3[,4]==Valor_S $[3]), 5])$

prop_4<-data.frame(prop.table(table(Imagem_treino [ , 4] , X [ , 1] , X [ , 2], X [ , 3] , X $[, 4]), 2: 5))$

prop_u4<-prop_4[which(is .nan(prop_4 [, "Freq"])!=TRUE), ]

p_util4<-as.vector (prop_u4[which(prop_u4[,2]==Valor_S[1] \& prop_u4[,3]==

Valor_S [2] \& prop_u4 $\overline{4}, 4]==$ Valor_S $[3] \&$ prop_u4[, $\overline{5}]==$ Valor_S $[\overline{4}]), 6])$

prop_5<-data.frame (prop.table(table(Imagem_treino [ , 4], X [ , 1], X [ , 2], X [ , 3], X $[, 4], \mathrm{X}[, 5]), 2: 6)$ )

prop_u $5<-$ prop_5[which(is .nan(prop_5[, "Freq"])!=TRUE), ]

p_util5<-as.vector (prop_u5[which(prop_u5[,2]== Valor_S[1] \& prop_u5 $[, 3]==$ Valor_S [2] \& prop_u5[,4]== Valor_S $[3] \&$ prop_u5[,5]==Valor_S $[4] \&$ prop_us[,6]==Valor_S $[5]), 7]$ )

prop_6<-data.frame(prop.table (table(Imagem_treino [ ,4] , X [ , 1] , X [ , 2], X [ , 3], X $[, 4], \mathrm{X}[, 5], \mathrm{X}[, 6]), 2: 7))$

prop_u6<-prop_6[which(is.nan(prop_6[, "Freq"])!=TRUE), ]

__util6<-as.vector (prop_u6[which(prop_u6[,2]==Valor_S[1] \& prop_u6[,3]==

Valor_S [2] \& prop_u6[,4]==Valor_S $[3] \&$ prop_u6[, 5$]==$ Valor_S[4] \&

prop_u6[,6]==Valor_S[5] \& prop_u6[,7]==Valor_S[6]),8])

prop_ $7<-$ data.frame (prop.table (table (Imagem_treino [ , 4] , X [ , 1] , X [ ,2] , X [ , 3] , X $[, 4], \mathrm{X}[, 5], \mathrm{X}[, 6], \mathrm{X}[, 7]), 2: 8))$

prop_u $7<-$ prop_7 [which (is .nan (prop_7 [, "Freq"])!=TRUE) ,]

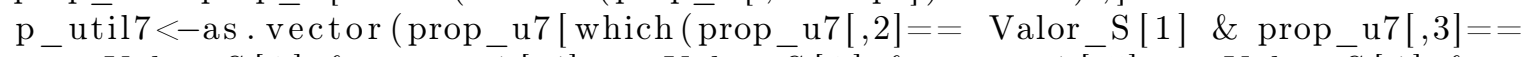

Valor_S [2] \& prop_u7[,4]== Valor_S [3] \& prop_u7[,5]== Valor_S $[4] \&$ prop_u7 $[, 6]==$ Valor_S $[5] \&$ prop_u7 $[, 7]==$ Valor_S $[6] \&$ prop_u7[,8]==

Valor_S $[7]), 9])$ 
805

prop_8<-data.frame (prop.table (table (Imagem_treino [ , 4] , X [ , 1], X [ , 2], X[, 3] , X $[, 4], \mathrm{X}[, 5], \mathrm{X}[, 6], \mathrm{X}[, 7], \mathrm{X}[, 8]), 2: 9))$

prop u $8<-$ prop_ 8 [which (is .nan (prop_8[, "Freq"])!=TRUE), ]

p_util8<-as_vector (prop_u8[which(prop_u8[,2]==Valor_S[1] \& prop_u8[,3]==

Valor_S[2] \& prop_u8[,4]==Valor_S[3] \& prop_u8[,5]==Valor_S[4] \& prop_u8 $[, 6]==$ Valor_S $[5] \&$ prop_u $8[, 7]==$ Valor_S $[6] \&$ prop_u8 $[, 8]==$ Valor_S [7] \& prop_u8[,9]==Valor_S [8]),10])

prop_9<-data.frame(prop.table(table(Imagem_treino [ , 4] , X [ , 1] , X [ , 2], X [ , 3] , X $[, 4], \mathrm{X}[, 5], \mathrm{X}[, 6], \mathrm{X}[, 7], \mathrm{X}[, 8], \mathrm{X}[, 9]), 2: 10))$

prop_u $9<-$ prop_9[which (is .nan (prop_9[, "Freq"])!=TRUE),

p_util9<-as.vector $($ prop_u9[which (prop_u9[,2]==Valor_S[1] \& prop_u9[,3]== Valor_S [2] \& prop_u9 $[, 4]==$ Valor_S $[3] \&$ prop_u $\overline{9}[, 5]==$ Valor_S $[4] \&$ prop_u9 $[, 6]==$ Valor_S [5] \& prop_u9 $[, 7]==$ Valor_S [6] \& prop_u9 $[, 8]==$ Valor_S $[7] \&$ prop_u9[,9]==Valor_S [8] \& prop_u9 $[, 10]==$ Valor_S $[9]), 11])$

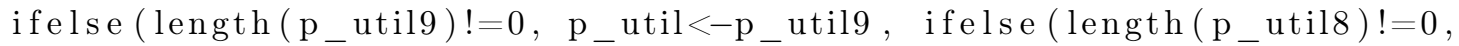
p_util $<-p \_u t i l 8$, ifelse $\left(\right.$ length $\left(p_{-}\right.$util $) !=0, p_{-}$util $<-p \_u t i l 7$, ifelse (

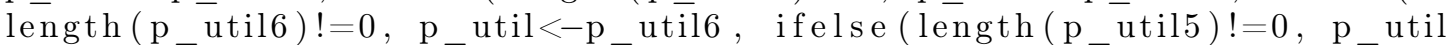
$<-p_{-}$util5, ifelse (length $\left(p_{-}\right.$util4) $)=0, p_{-}$util $<-p_{-}$util4, ifelse (length ( $p_{-}$util3 $) !=0, p_{-}$util $<-p_{-} u t i l 3$, ifelse $\left(\operatorname{length}\left(p_{-}\right.\right.$util 2$) !=0, p_{-} u t i l<-$

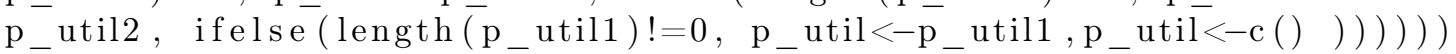
$\overline{)})$

ifelse $\left(\right.$ length $\left(p_{-}\right.$util $)==0$, conta $9<-\operatorname{conta} 9+1,\{$

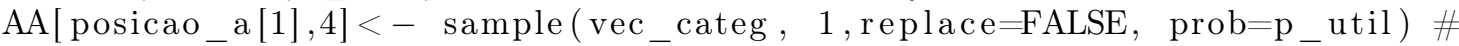
estimativa de novos valores

AA[posicao_a[1],21]<-p_util[1] \# a probabilidade não condicional utilizada para estimativa da categoria 1: Level

$\mathrm{AA}[$ posicao_a[1],22]<- p_util[2] \# a probabilidade não condicional utilizada para estimativa da categoria 2: Level

AA[posicao_a[1],23]<-p_util[3] \# a probabilidade não condicional utilizada para estimativa da categoria 3: Level

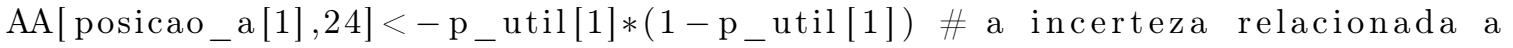
categoria 1: Level

$\mathrm{AA}[$ posicao_a[1],25]<-p_util[2]*(1-p_util[2]) \# a incerteza relacionada a categoria 2: Level

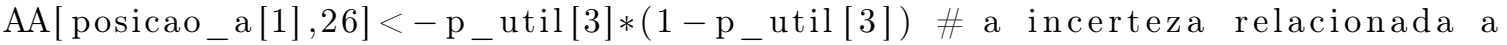
categoria 3: Level

AA[posicao_a $[1], 6]<-$ Nivel_prob \# Level

AA [posicao_a [1],7]<-Valor_S [1]

$\mathrm{AA}[$ posicao_a $[1], 8]<-$ Valor_S $[2]$

$\mathrm{AA}[$ posicao_a $[1], 9]<-$ Valor_S $[3]$

$\mathrm{AA}[$ posicao_a $[1], 10]<-$ Valor_S $[4]$

$\mathrm{AA}[$ posicao_a $[1], 11]<-$ Valor_S [5]

AA [ posicao_a $[1], 12]<-$ Valor_S [6]

AA [ posicao_a $[1], 13]<-$ Valor_S $[7]$

$\mathrm{AA}[$ posicao_a $[1], 14]<-$ Valor_S [8]

AA [ posicao_a [1],15]<-Valor_S [9]

AA [ posicao_a [1],31]<-posto_S [1]

AA [ posicao_a $[1], 32]<-$ posto_S $[2]$

$\mathrm{AA}[$ posicao_a $[1], 33]<-$ posto_S $[3]$

$\mathrm{AA}[$ posicao_a $[1], 34]<-$ posto_S $[4]$

$\mathrm{AA}[$ posicao_a $[1], 35]<-$ posto_S $[5]$

$\mathrm{AA}[$ posicao_a $[1], 36]<-$ posto_S $[6]$

$\mathrm{AA}[$ posicao_a $[1], 37]<-$ posto_S $[7]$

$\mathrm{AA}[$ posicao_a $[1], 38]<-$ posto_S $[8]$

$\mathrm{AA}[$ posicao_a $[1], 39]<-$ posto_S $[9]$ 
833

834

835

836

837 )

838

839

840

841

842

843

844

845

846

847

848

849

850

851

852

853

854

855

856

857

858

859

860

861

862

863

864

865

866

867

868

869

870

871

872

873

874

posicao_a $<-$ posicao_a $[-1]$

$$
\}
$$

\}

, if else (Nivel_prob $==10,\{$

prop_1<-data.frame (prop.table (table (Imagem_treino $[, 4], X[, 1]), 2: 2))$

prop_u1<-prop_1 [which (is . nan (prop_1 [, "Freq"] )!=TRUE),]

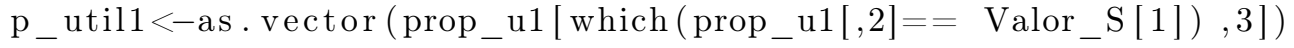

prop_2<-data.frame (prop.table (table (Imagem_treino [,4],X[,1],X[,2]),2:3))

prop_u $2<-$ prop_2[which (is . nan (prop_2 [, "Freq"] $) !=$ TRUE) , ]

p_util $2<-$ as .vector $\left(\operatorname{prop}_{-} u 2\left[\right.\right.$ which $\left(\overline{p r o p}_{-} \mathrm{u} 2[, 2]==\right.$ Valor_S $[1] \&$ prop_u2 $[, 3]==$ Valor_S [2]),4])

prop_3<-data.frame (prop.table (table (Imagem_treino [,4],X[,1],X[,2],X[, 3$])$ $, 2: 4))$

prop_u $3<-$ prop_3 [which ( is . nan (prop_3 [, "Freq"])!=TRUE) ,]

p_util3<-as.vector (prop_u3 [which (prop_u3[,2]==Valor_S $[1]$ \& prop_u3 $[, 3]==$ Valor_S[2] \& prop_u3[,4]==Valor_S [3]), 5])

prop_4<-data.frame (prop.table (table (Imagem_treino [, 4],X[, 1], X [ , 2] , X [ , 3], X $[, 4]), 2: 5))$

prop_u4<-prop_4 [which (is . nan (prop_4 [, "Freq"])!=TRUE) ,]

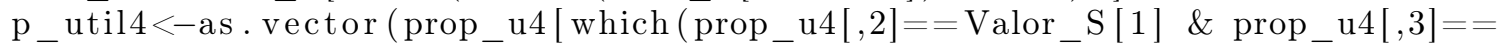

Valor_S [2] \& prop_u4[,4]==Valor_S [3] \& prop_u4[, $\overline{5}]==$ Valor_S $[4]), 6])$

prop_5<-data.frame (prop.table (table (Imagem_treino [,4],X[, 1], X [ , 2] , X [ , 3], X $[, 4], \mathrm{X}[, 5]), 2: 6))$

prop_u $5<-$ prop_5[which ( is . nan (prop_5 [, "Freq"])!=TRUE) ,]

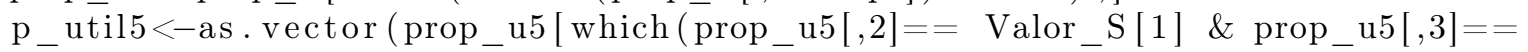
Valor_S [2] \& prop_u5[,4]== Valor_S $[3]$ \& prop_u5[,5]==Valor_S $[4] \&$ prop_ $_{-} \overline{\mathrm{s}}[, 6]==$ Valor_S $\left.\left.\left.[5]\right), 7\right]\right)$

prop_6<-data.frame (prop.table (table (Imagem_treino [,4],X[, 1], X [ , 2] , X [, 3], X $[, 4], \mathrm{X}[, 5], \mathrm{X}[, 6]), 2: 7))$

prop_u6<-prop_6 [which ( is . nan (prop_6 [, "Freq"])!=TRUE) ,]

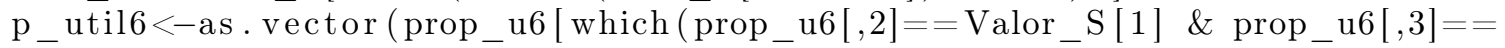

Valor_S [2] \& prop_u6[,4]==Valor_S [3] \& prop_u6[, 5$]==$ Valor_S $[4] \&$

prop_u $6[, 6]==$ Valor_S $[5] \&$ prop_u$_{-} 6[, 7]==$ Valor_S[6]) ,8])

prop_ $7<$-data.frame (prop.table (table (Imagem_treino [, 4], X[, 1], X[ , 2], X [ , 3], X $[, 4], \mathrm{X}[, 5], \mathrm{X}[, 6], \mathrm{X}[, 7]), 2: 8))$

prop_u7<-prop_7 [which (is . nan (prop_7 [, "Freq"])!=TRUE) ,]

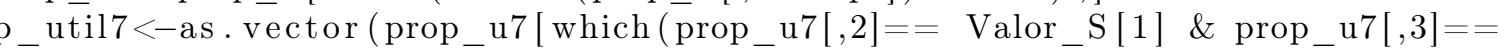

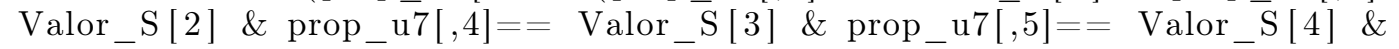

prop_u $\overline{7}[, 6]==$ Valor_S $\left.[5] \& \operatorname{prop}_{-} \mathrm{u} 7 \overline{[}, 7\right]==$ Valor_S $[6] \&$ prop_u $7[\overline{, 8}]==$

Valor_S [7]),9])

prop_8<-data.frame (prop.table (table (Imagem_treino [,4],X[, 1], X [ ,2] , X [, 3], X $[, 4], \mathrm{X}[, 5], \mathrm{X}[, 6], \mathrm{X}[, 7], \mathrm{X}[, 8]), 2: 9))$

prop_u $8<-$ prop_8 [which $\left(\right.$ is . nan $\left(\operatorname{prop}_{-} 8[\right.$, "Freq" ])!=TRUE) ,]

p_util8<-as.vector (prop_u8[which (prop_u8[,2]==Valor_S $[1]$ \& prop_u8 $[, 3]==$

Valor_S [2] \& prop_u8[,4]==Valor_S [3] \& prop_u8[, 5$]==$ Valor_S $[4]$ \& 
prop_u8 $[, 6]==$ Valor_S $[5] \&$ prop_u8 $[, 7]==$ Valor_S $[6] \&$ prop_u8 $[, 8]==$ Valor_S [7] \& prop_u8[,9]==Valor_S [8]),10])

875

886

887 ifelse $($ length $(\mathrm{p}$ util $)==0$, conta $10<-\operatorname{conta} 10+1,\{$ $[, 4], \mathrm{X}[, 5], \mathrm{X}[, 6], \mathrm{X}[, 7], \mathrm{X}[, 8], \mathrm{X}[, 9]), 2: 10))$

prop_u $9<-$ prop_9 [which (is .nan (prop_9 [, "Freq"])!=TRUE) ,]

$\overline{\mathrm{X}}[, 4], \mathrm{X}[, 5], \mathrm{X}[, 6], \mathrm{X}[, 7], \mathrm{X}[, 8], \mathrm{X}[, 9], \mathrm{X}[, 1 \overline{0}]), 2: 11))$

prop_u10<-prop_10[which(is .nan(prop_10 [, "Freq"])!=TRUE) ,]

[9] \& prop_u10[,11]==Valor_S[10]),12]) $p_{-}$util 1$\left.\left.\left.\left.\left.\left.\left.\left.) !=0, p_{-} u t i l<-p_{-} u t i l 1, p_{-} u t i l<-c(())\right)\right)\right)\right)\right)\right)\right)\right)$ estimativa de novos valores utilizada para estimativa da categoria 1: Level utilizada para estimativa da categoria 2: Level utilizada para estimativa da categoria 3 : Level categoria 1: Level categoria 2: Level categoria 3: Level

AA $[$ posicao_a $[1], 6]<-$ Nivel_prob \# Level

$\mathrm{AA}[$ posicao_a $[1], 7]<-$ Valor_S $[1]$

$\mathrm{AA}[$ posicao_a $[1], 8]<-$ Valor_S $[2]$

AA [ posicao_a $[1], 9]<-$ Valor_S $[3]$

AA $[$ posicao_a $[1], 10]<-$ Valor_S $[4]$

$\mathrm{AA}[$ posicao_a [1],11] $<-$ Valor_S [5]

$\mathrm{AA}[$ posicao_a $[1], 12]<-$ Valor_S $[6]$

AA [ posicao_a [1],13]<-Valor_S [7]

AA $[$ posicao_a $[1], 14]<-$ Valor_S $[8]$

AA[ posicao_a $[1], 15]<-$ Valor_S $[9]$

AA[posicao_a $[1], 16]<-$ Valor_S [10]

$\mathrm{AA}[$ posicao_a $[1], 31]<-$ posto_S $[1]$

$\mathrm{AA}[$ posicao_a $[1], 32]<-$ posto_S $[2]$

AA [ posicao_a $[1], 33]<-$ posto_S $[3]$

AA $[$ posicao_a $[1], 34]<-$ posto_S $[4]$

prop_9<-data.frame(prop.table(table(Imagem_treino [ , 4] , X [ , 1] , X [ , 2], X [ , 3], X

p_util9<-as .vector $\left(\operatorname{prop}_{-} u 9\left[\right.\right.$ which $\left(\operatorname{prop}_{-} \mathrm{u} 9[, 2]==\right.$ Valor_S [1] \& prop_u9[,3] $==$

Valor_S[2] \& prop_u9 $[, 4]==$ Valor_S $[3] \&$ prop_u9[,5]==Valor_S[4] \&

prop_u9 $[, 6]==$ Valor_S [5] \& prop_u9 $[, 7]==$ Valor_S [6] \& prop_u9[,8] $==$ Valor_S $[7] \&$ prop_u9 $[, 9]==$ Valor_S [8] \& prop_u9 $[, 10]==$ Valor_S $[9]), 11])$

prop_10<-data.frame (prop.table (table (Imagem_treino [ , 4], X [ , 1], X [ , 2], X[, 3],

p_util10<-as.vector (prop_u10[which(prop_u10[,2]==Valor_S $[1] \&$ prop_u10

$[, 3]==$ Valor_S $[2] \&$ prop_u10[,4]==Valor_S [3] \& prop_u10[,5]==Valor_S [4]

\& prop_u10[,6]==Valor_S $[5]$ \& prop_u10[,7]==Valor_S $[6] \&$ prop_u10

$[, 8]==$ Valor_S $[7] \&$ prop_u10[,9]==Valor_S $[8] \&$ prop_u10[,10] $=\overline{=}$ Valor_S

ifelse $\left(\right.$ length $\left(p \_u t i l 10\right) !=0, \quad p \_u t i l<-p \_u t i l 10$, ifelse $\left(\right.$ length $\left(p \_u t i l 9\right) !=0$,

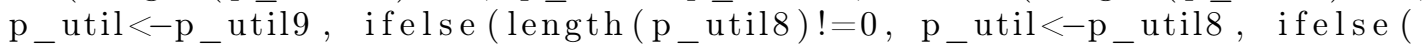
length $\left(p_{-}\right.$util $) !=0, p_{-}$util $<-p_{-}$util7, ifelse $\left(\right.$length $\left(p_{-}\right.$util6 $) !=0, p_{-}$util $<-p_{-}$util6, ifelse (length $\left(p_{-}\right.$util 5$) !=0, p_{-}$util $<-p_{-}$util5, ifelse (length ( $\left.p_{-} u t i l 4\right) !=0, p_{-} u t i l<-p_{-} u t i l 4$, ifelse $\left(\right.$ length $\left(p_{-} u t i l 3\right) !=0, p_{-} u t i l<-$ $p_{-}$util3, ifelse $\left(\right.$length $\left(\bar{p}_{-}\right.$util $) !=0, p_{-}$util $<-p_{-}$util2, ifelse $($length (

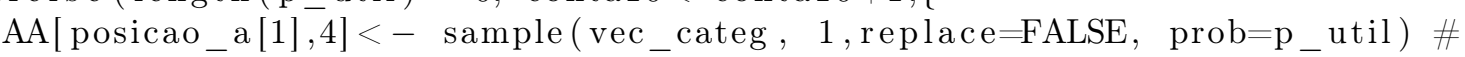

$\mathrm{AA}[$ posicao_a[1],21]<-p_util[1] \# a probabilidade não condicional

AA[posicao_a[1],22]<- p_util[2] \# a probabilidade não condicional

AA[posicao_a[1],23]<- p_util[3] \# a probabilidade não condicional

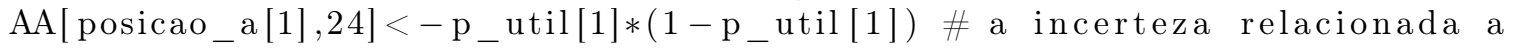

$\mathrm{AA}[$ posicao_a $[1], 25]<-\mathrm{p}_{\text {_ }}$ util $[2] *\left(1-\mathrm{p}_{-}\right.$util[2] $) \#$ a incerteza relacionada a

AA[posicao_a[1],26]<- p_util[3]*(1-p_util[3]) \# a incerteza relacionada a 
910 AA[posicao_a [1],35]<-posto_S [5]

911 AA[posicao_a $[1], 36]<-$ posto_S $[6]$

912 AA[posicao_a $[1], 37]<-$ posto_S $[7]$

913 AA[posicao_a [1],38]<-posto_S [8]

914 AA[posicao_a[1],39]<-posto_S [9]

915 AA[posicao_a [1],40]<-posto_S [10]

916 posicao_a $<-$ posicao_a $[-1]$

917

$918\}$

919 )

920

921

922

923

924

925

926

927

928

929

930

931

932

933

$$
\text { , if else (Nivel_prob }==11,\{
$$

prop_1<-data.frame (prop.table (table (Imagem_treino [,4],X[,1]),2:2))

prop_u1<-prop_1[which(is .nan (prop_1[, "Freq"])!=TRUE), ]

p_util1<-as.vector $\left(\operatorname{prop}_{-} u 1\left[\right.\right.$ which $\left(\operatorname{prop}_{-} u 1[, 2]==\right.$ Valor_S $\left.\left.\left.[1]\right), 3\right]\right)$

prop_2<-data.frame (prop.table (table (Imagem_treino [,4], X[, 1], X[,2] ),2:3))

prop_u2<-prop_2[which(is .nan (prop_2[, "Freq"])!=TRUE),]

p_util2 $<-$ as.vector $\left(\right.$ prop_u$_{-}\left[\right.$which $\left(\operatorname{prop}_{-} u 2[, 2]==\right.$ Valor_S $[1] \&$ prop_u2 $[, 3]==$ Valor_S $[2]), 4])$

prop_3<-data.frame (prop.table (table(Imagem_treino [ , 4] , X [ , 1] , X [ ,2] , X [ , 3]) $, 2: 4))$

prop_u3<-prop_3[which(is .nan (prop_3 [, "Freq"])!=TRUE) ,]

p_util3<-as.vector (prop_u3 [which(prop_u3 $[, 2]==$ Valor_S $[1] \&$ prop_u3 $[, 3]==$ Valor_S[2] \& prop_u3[,4]==Valor_S $[3]), 5])$

prop_4<-data.frame (prop.table(table(Imagem_treino [ , 4] , X [ , 1] , X [ , 2], X [ , 3], X $[, 4]), 2: 5))$

prop_u4<-prop_4[which(is .nan(prop_4 [, "Freq"])!=TRUE),]

p_util4<-as.vector (prop_u4[which(prop_u4[,2]==Valor_S[1]\& prop_u4[,3]==

Valor_S [2] \& prop_u4 $\overline{4}, 4]==$ Valor_S $[3] \&$ prop_u4[, $\overline{5}]==$ Valor_S $[\overline{4}]), 6])$

942

943

prop_5<-data.frame (prop.table (table (Imagem_treino [ , 4] , X [ , 1] , X [ ,2] , X [ , 3] , X $[, 4], \mathrm{X}[, 5]), 2: 6))$

944

945

prop_u $5<-$ prop_5[which (is .nan (prop_5 [, " Freq"])!=TRUE) ,]

p_util $5<-$ as. vector $\left(\operatorname{prop}_{-} u 5\left[\right.\right.$ which $\left(\operatorname{prop}_{-} u 5[, 2]==\right.$ Valor_S[1] \& prop_u5[,3]==

Valor_S [2] \& prop_u5[,4]== Valor_S $[3] \&$ prop_u5[,5]==Valor_S[4] \& prop_us $[, 6]==$ Valor_S $[5]), 7])$

946

947

948

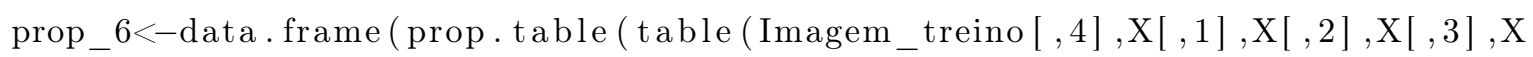
$[, 4], \mathrm{X}[, 5], \mathrm{X}[, 6]), 2: 7))$

949 prop_u6<-prop_6[which(is .nan (prop_6[, "Freq"])!=TRUE), ]

950 p_util6<-as.vector $\left(\operatorname{prop} \_u 6\left[\right.\right.$ which $\left(\operatorname{prop}_{-} u 6[, 2]==\right.$ Valor_S[1] \& prop_u6[,3]== Valor_S [2] \& prop_u6[,4]==Valor_S[3] \& prop_u6[, $\overline{5}]==$ Valor_S[4] \& prop_u6[,6]==Valor_S[5] \& prop_u6[,7]==Valor_S[6]),8])

951

952 prop_ $7<-$ data.frame (prop.table (table (Imagem_treino $[, 4], \mathrm{X}[, 1], \mathrm{X}[, 2], \mathrm{X}[, 3], \mathrm{X}$ $[, 4], \mathrm{X}[, 5], \mathrm{X}[, 6], \mathrm{X}[, 7]), 2: 8))$

953 prop_u7<-prop_7[which (is . nan $\left(\operatorname{prop}_{-} 7[\right.$, "Freq" ])!=TRUE) ,]

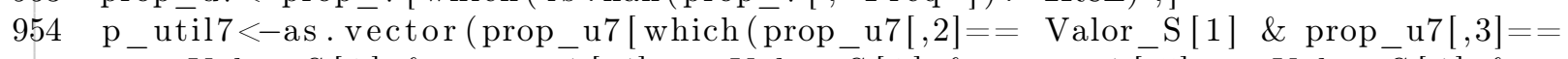
Valor_S [2] \& prop_u7 [,4]== Valor_S [3] \& prop_u7[,5]== Valor_s $[4] \&$

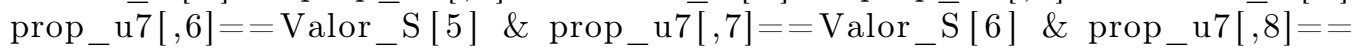


955

956

957

958

959

960

961

962

963

964

965

966

967

968

969

970

971

972

973

974

975

976

977

978

979

980

981

Valor_S [7]),9])

prop_8<-data.frame (prop.table (table (Imagem_treino [,4],X[, 1], X [ ,2], X[, 3], X $[, 4], \mathrm{X}[, 5], \mathrm{X}[, 6], \mathrm{X}[, 7], \mathrm{X}[, 8]), 2: 9))$

prop_u $8<-$ prop_8[which (is . nan (prop_8 [, "Freq"])!=TRUE), ]

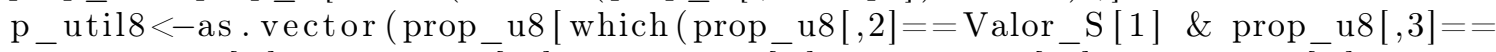

Valor_S [2] \& prop_u8[,4]==Valor_S [3] \& prop_u8[, $\overline{5}]==$ Valor_S $[4] \&$

prop_u $8[, 6]==$ Valor_S $[5] \& \operatorname{prop}_{-} \mathrm{u} 8[, 7]==$ Valor_S $[6] \& \operatorname{prop}_{-} \overline{\mathrm{u}} 8[, 8]==$

Valor_S [7] \& prop_u8[,9]==Valor_S[8]),10])

prop_9<-data.frame (prop.table (table (Imagem_treino [,4],X[,1],X[, 2] ,X [, 3], X $[, 4], \mathrm{X}[, 5], \mathrm{X}[, 6], \mathrm{X}[, 7], \mathrm{X}[, 8], \mathrm{X}[, 9]), 2: 10 \overline{)})$

prop_u $9<-$ prop_9 [which (is . nan (prop_9 [, "Freq"])!=TRUE) ,]

p_util9<-as.vector $\left(\operatorname{prop}_{-}\right.$u9 $\left[\right.$which $\left(\overline{p r o p}_{-} u 9[, 2]==\right.$ Valor_S $[1] \quad$ \& prop_u9 $[, 3]==$

Valor_S [2] \& prop_u9[,4]==Valor_S [3] \& prop_u $\overline{9}[, 5]==$ Valor_S $[4] \&$

prop_u9[,6]==Valor_S[5] \& prop_u9[,7]==Valor_S[6] \& prop_u9[,8]==

Valor_S [7] \& prop_u $9[, 9]==$ Valor_S [8] \& prop_u9 $[, 10]==$ Valor_S [9]),11])

prop_10<-data.frame (prop.table (table (Imagem_treino [,4],X[, 1], X[, 2] , X [ , 3] ,

$\overline{\mathrm{X}}[, 4], \mathrm{X}[, 5], \mathrm{X}[, 6], \mathrm{X}[, 7], \mathrm{X}[, 8], \mathrm{X}[, 9], \mathrm{X}[, 1 \overline{0}]), 2: 11))$

prop_u10<-prop_10 [which ( is . nan (prop_10 [, "Freq"])!=TRUE) ,]

p_util10<-as.vector (prop_u10[which (

$[, 3]==$ Valor_S [2] \& prop_u10[,4]==Valor_S [3] \& prop_u10[,5]==Valor_S [4]

\& prop_u10[,6]==Valor_S $[5]$ \& prop_u10[,7]==Valor_S [6] \& prop_u10

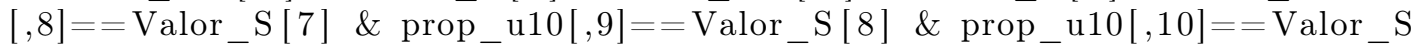

[9] \& prop_u10[,11]==Valor_S[10]),12])

prop_11<-data.frame (prop.table (table (Imagem_treino [,4],X[, 1],X[,2],X[, 3] ,

$\overline{\mathrm{X}}[, 4], \mathrm{X}[, 5], \mathrm{X}[, 6], \mathrm{X}[, 7], \mathrm{X}[, 8], \mathrm{X}[, 9], \mathrm{X}[, 1 \overline{0}], \mathrm{X}[, 11]), 2: 12))$

prop_u11<-prop_11 [which (is .nan (prop_11 [, "Freq"])!=TRUE),]

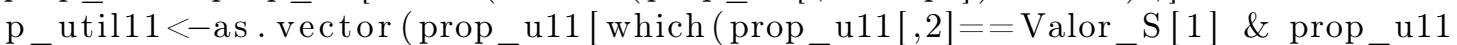

$[, 3]==$ Valor_S [2] \& prop_u11[,4]==Valor_S [3] \& prop_u11[,5]==Valor_S [4]

\& prop_u11[, 6$]==$ Valor_S $[5]$ \& prop_u11[,7]==Valor_S $[6]$ \& prop_u11

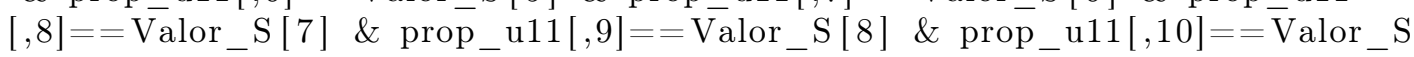

[9] \& prop_u11[,11]==Valor_S $[10] \& \operatorname{prop}_{-} \mathrm{u} 11[, 12]==\overline{\text { Valor }}$ _S $\left.\left.\left.[11]\right), 13\right]\right)$

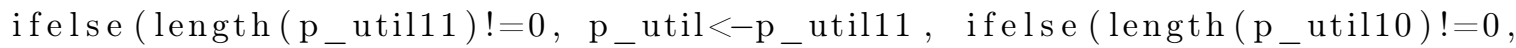
$p_{-}$util $<-p_{-}$util10, if else $\left(\bar{l} \operatorname{ength}\left(p_{-}\right.\right.$util9 $) !=0, p_{-}$util $<-p_{-}$util9, ifelse ( length $\left(p_{-}\right.$util8) $!=0, p_{-}$util $<-p_{-}$utils, if else $\left(\right.$length $\left(p_{-}\right.$util $) !=0, p_{-}$util $<-p_{-}$util7,,$\quad$ ifelse $\left(\operatorname{length}\left(p_{-}\right.\right.$util6) $!=0, p_{-}$util $<-p_{-}$util6,,$\quad$ ifelse $(\operatorname{length}($

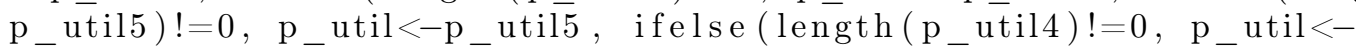
p_util4, ifelse ( length $\left(\bar{p}_{-}\right.$util3 $) !=0, p_{-}$util $<-p_{-} \bar{u}_{\text {til }}$, if else ( length (

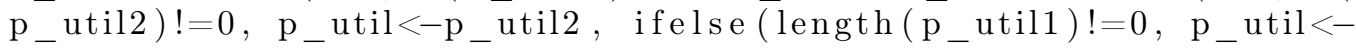
$\left.\left.\left.\left.\left.\left.\left.p_{-} u t i l 1, p_{-} u t i l<-c(),\right)\right)\right)\right)\right)\right)\right)$ )) )

if else $\left(\right.$ length $\left(p_{-}\right.$util $)==0$, conta $11<-$ conta $11+1,\{$

$\mathrm{AA}[$ posicao_a[1],4] $<-$ sample (vec_categ, 1 , replace=FALSE, prob=p_util) \# estimativa de novos valores

$\mathrm{AA}[$ posicao_a[1],21]<-p_util[1] \# a probabilidade não condicional utilizada para estimativa da categoria 1: Level

$\mathrm{AA}[$ posicao_a[1],22]<- p_util[2] \# a probabilidade não condicional utilizada para estimativa da categoria 2: Level

AA[posicao_a[1],23] $<-$ p_util [3] \# a probabilidade não condicional utilizada para estimativa da categoria 3: Level

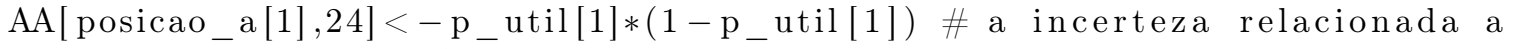
categoria 1: Level

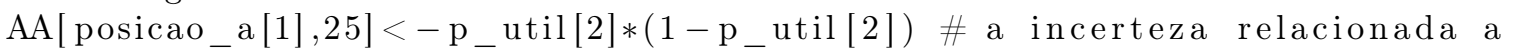
categoria 2: Level 
982 AA[posicao_a[1],26]<-p_util[3]*(1-p_util[3]) \# a incerteza relacionada a categoria 3: Level

983 AA[posicao_a $[1], 6]<-$ Nivel_prob \# Level

984 AA[posicao_a $[1], 7]<-$ Valor_S $[1]$

985 AA[ posicao_a [1],8]<-Valor_S [2]

986 AA[ posicao_a [1],9]<-Valor_S [3]

987 AA[posicao_a $[1], 10]<-$ Valor_S $[4]$

988 AA[posicao_a $[1], 11]<-$ Valor_S $[5]$

989 AA[posicao_a [1],12]<-Valor_S [6]

990 AA[posicao_a $[1], 13]<-$ Valor_S [7]

991 AA[posicao_a[1],14]<-Valor_S [8]

992 AA[posicao_a $[1], 15]<-$ Valor_S $[9]$

993 AA [ posicao_a [1],16]<-Valor_S [10]

994 AA[posicao_a [1],17]<-Valor_S [11]

995 AA[posicao_a $[1], 31]<-$ posto_S [1]

996 AA[posicao_a $[1], 32]<-$ posto_S $[2]$

997 AA[posicao_a [1],33]<- posto_S [3]

998 AA[posicao_a $[1], 34]<-$ posto_S $[4]$

999 AA[posicao_a $[1], 35]<-$ posto_S $[5]$

1000 AA[posicao_a $[1], 36]<-$ posto_S $[6]$

1001 AA[posicao_a [1],37]<-posto_S [7]

1002 AA[posicao_a [1],38]<-posto_S [8]

1003 AA[posicao_a[1],39]<-posto_S [9]

1004 AA[posicao_a $[1], 40]<-$ posto_S $[10]$

1005 AA[posicao_a $[1], 41]<-$ posto_S [11]

1006 posicao_a $<-$ posicao_a $[-1]$

1007

$1008\}$

1009 )

1010

1011

1012

1013

1014

1015

1016

1017

1018

1019

1020

1021

1022

\}

, if else (Nivel_prob $==12,\{$

prop_1<-data.frame (prop.table (table(Imagem_treino [,4],X[,1]),2:2))

prop_u1<-prop_1[which(is .nan(prop_1[, "Freq"] $) !=$ TRUE), ]

p_util1<-as.vector $\left(\operatorname{prop} \_u 1\left[\right.\right.$ which $\left(\operatorname{prop}_{-} u 1[, 2]==\right.$ Valor_S $\left.\left.\left.[1]\right), 3\right]\right)$

prop_2<-data.frame (prop.table (table (Imagem_treino [ , 4], X [ , 1], X [ ,2] ) ,2:3) )

prop_u $2<-$ prop_2[which(is .nan (prop_2[, "Freq"] $) !=$ TRUE) ,]

p_util2<-as.vector $\left(\right.$ prop_u2 $\left[\right.$ which $\left(\operatorname{prop}_{-} u 2[, 2]==\right.$ Valor_S $[1] \&$ prop_u2 $[, 3]==$ Valor_S [2]),4])

prop_3<-data.frame(prop.table(table (Imagem_treino [, 4], X[, 1], X [, 2], X [ , 3]) $, 2: 4))$

1023

1024

1025

1026

1027

1028

1029

1030

1031

1032

prop_u3<-prop_3[which(is .nan(prop_3[, "Freq"])!=TRUE), ]

p_util3<-as.vector (prop_u3 [which(prop_u3 $[, 2]==$ Valor_S $[1] \&$ prop_u3 $[, 3]==$ Valor_S [2] \& prop_u3[,4]==Valor_S [3] $), 5]$ )

prop_4<-data.frame (prop.table (table(Imagem_treino [ , 4] , X [ , 1] , X [ , 2], X [ , 3], X $[, 4]), 2: 5)$ )

prop_u4<-prop_4[which(is.nan(prop_4[, "Freq"])!=TRUE), ]

$p_{-} u t i l 4<-$ as . vector $\left(\operatorname{prop}_{-} u 4\left[\right.\right.$ which $\left(\operatorname{prop}_{-} u 4[, 2]==\right.$ Valor_S $[1] \&$ prop_u4[,3]==

Valor_S [2] \& prop_u4[,4]==Valor_S [3] \& prop_u4[, $\overline{5}]==$ Valor_S $[4]), 6])$

prop_5<-data.frame(prop.table(table(Imagem_treino [ , 4] , X [ , 1] , X [ , 2], X [ , 3] , X $[, 4], \mathrm{X}[, 5]), 2: 6))$

1033 prop_u $5<-$ prop_5[which(is .nan(prop_5[, "Freq"])!=TRUE) ,] 
1034

1035

1036

1037

1038

1039

1040

1041

1042

1043

1044

1045

1046

1047

1048

1049

1050

1051

1052

1053

1054

1055

1056

1057

1058

1059

1060

1061

1062

1063 p_util5<-as . vector $\left(\operatorname{prop}_{-} \mathrm{u} 5\left[\right.\right.$ which $\left(\operatorname{prop}_{-} \mathrm{u} 5[, 2]==\right.$ Valor_S $[1]$ \& prop_u5[,3]== Valor_S [2] \& prop_u5[,4]==Valor_S [3] \& prop_u5[,5]==Valor_S [4] \& prop_u $\overline{5}[, 6]==$ Valor_S $[5]), 7])$

prop_6<-data.frame (prop.table (table (Imagem_treino [,4],X[,1],X[,2],X[,3],X $[, 4], \mathrm{X}[, 5], \mathrm{X}[, 6]), 2: 7))$

prop_u6<-prop_6 [which (is . nan (prop_6 [, "Freq"])!=TRUE) ,]

p_util6<-as.vector (prop_u6[which (prop_u6[,2]==Valor_S [1] \& prop_u6[,3]==

Valor_S [2] \& prop_u6[,4]==Valor_S [3] \& prop_u6[, 5$]==$ Valor_S $[4] \&$ prop_u6[,6]==Valor_S[5] \& prop_u6[,7]==Valor_S[6]) ,8])

prop_ $7<$-data.frame (prop.table (table (Imagem_treino [, 4],X[, 1], X[, 2] ,X [, 3], X $[, 4], \mathrm{X}[, 5], \mathrm{X}[, 6], \mathrm{X}[, 7]), 2: 8))$

prop_u $7<-$ prop_7 [which $($ is .nan (prop_7 [, "Freq"])!=TRUE) ,]

p_util7<-as.vector $\left(\operatorname{prop}_{-} u 7\left[\right.\right.$ which $\left(\overline{p r o p}_{-} \mathrm{u} 7[, 2]==\right.$ Valor_S [1] \& prop_u7 [,3] $==$

Valor_S [2] \& prop_u7 $[, 4]==$ Valor_S [3] \& prop_u7[,5]== Valor_S [4] \&

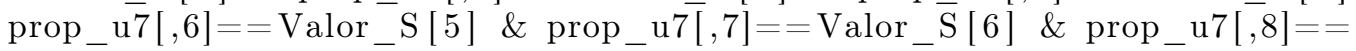
Valor_S[7]),9])

prop_8<-data.frame (prop.table (table (Imagem_treino [,4],X[,1],X[,2],X[,3],X $[, 4], \mathrm{X}[, 5], \mathrm{X}[, 6], \mathrm{X}[, 7], \mathrm{X}[, 8]), 2: 9))$

prop_u $8<-$ prop_8 [which (is . nan (prop_8 [, "Freq"])!=TRUE) ,]

p_util $8<-$ as.vector (prop_u8 [which $\left(\overline{p r o p}_{-} \mathrm{u} 8[, 2]==\right.$ Valor_S $[1]$ \& prop_u $8[, 3]==$

Valor_S [2] \& prop_u8[,4]==Valor_S [3] \& prop_u8[,5]==Valor_S [4] \&

prop_u $8[, 6]==$ Valor_S $[5]$ \& prop_u $8[, 7]==$ Valor_S $[6]$ \& prop_u $8[, 8]==$

Valor_S [7] \& prop_u8[,9]==Valor_S [8]),10])

prop_9<-data.frame (prop. table (table (Imagem_treino [,4],X[,1],X[, 2], X [, 3] , X $[, 4], \mathrm{X}[, 5], \mathrm{X}[, 6], \mathrm{X}[, 7], \mathrm{X}[, 8], \mathrm{X}[, 9]), 2: 1 \overline{0}))$

prop_u $9<-$ prop_9 [which (is . nan (prop_9 [, "Freq"])!=TRUE) ,]

p_util9<-as.vector (prop_u9[which(prop_u9[,2]==Valor_S [1] \& prop_u9[,3]==

Valor_S [2] \& prop_u $[, 4]==$ Valor_S $[3] \&$ prop_u$_{-}[, 5]==$ Valor_S $[4] \&$

prop_u9[,6]==Valor_S[5] \& prop_u9[,7]==Valor_S[6] \& prop_u9[,8]==

Valor_S[7] \& prop_u $9[, 9]==$ Valor_S [8] \& prop_u9 $[, 10]==$ Valor_S [9]) , 11])

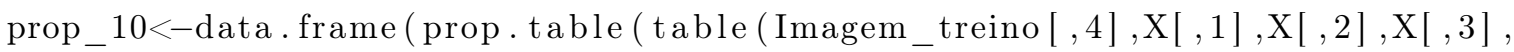

$\overline{\mathrm{X}}[, 4], \mathrm{X}[, 5], \mathrm{X}[, 6], \mathrm{X}[, 7], \mathrm{X}[, 8], \mathrm{X}[, 9], \mathrm{X}[, 1 \overline{0}]), 2: 11))$

prop_u $10<-$ prop_10 [which ( is .nan (prop_10 [, "Freq"])!=TRUE),]

p_util10<-as.vector (prop_u10[which ( $\operatorname{prop}_{-}$u10[,2]==Valor_S [1] \& prop_u10

$[, 3]==$ Valor_S [2] \& prop_u10[,4]==Valor_S $[3] \quad \&$ prop_u10[,5]== Valor_S [4]

\& prop_u10[,6]==Valor_S $[5]$ \& prop_u10[,7]==Valor_S $[6]$ \& prop_u10

$[, 8]==$ Valor_S $[7] \quad \&$ prop_u10[,9]==Valor_S [8] \& prop_u10[,10]= = Valor_S

[9] \& prop_u10[,11]==Valor_S[10]),12])

prop_11<-data.frame (prop.table (table (Imagem_treino [,4],X[,1],X[,2], X[, 3] ,

$\overline{\mathrm{X}}[, 4], \mathrm{X}[, 5], \mathrm{X}[, 6], \mathrm{X}[, 7], \mathrm{X}[, 8], \mathrm{X}[, 9], \mathrm{X}[, 1 \overline{0}], \mathrm{X}[, 11]), 2: 12))$

prop_u11<-prop_11[which (is .nan (prop_11 [, "Freq"])!=TRUE),]

p_util11<-as.vector (prop_u11[which (prop_u11[,2]==Valor_S[1] \& prop_u11

$[, 3]==$ Valor_S [2] \& prop_u11[,4]==Valor_S [3] \& prop_u11[,5]==Valor_S [4]

\& prop_u11[,6]==Valor_s $[5]$ \& prop_u11[,7]==Valor_S $[6]$ \& prop_u11

$[, 8]==$ Valor_S $[7] \&$ prop_ $_{-} \mathrm{u} 11[, 9]==\bar{V}_{\text {alor }} \mathrm{S}[8] \& \operatorname{prop}_{-} \mathrm{u} 11[, 10]==\overline{\mathrm{V}}$ alor $\mathrm{S}$

[9] \& prop_u11[,11]==Valor_S[10] \& prop_u11[,12]== Valor_S $[11]), 13])$

prop_12<-data.frame (prop.table (table (Imagem_treino [,4],X[, 1], X [, 2], X[ , 3] ,

$\overline{\mathrm{X}}[, 4], \mathrm{X}[, 5], \mathrm{X}[, 6], \mathrm{X}[, 7], \mathrm{X}[, 8], \mathrm{X}[, 9], \mathrm{X}[, 1 \overline{0}], \mathrm{X}[, 11], \mathrm{X}[, 12]), 2: 13))$

prop_u12<-prop_12 [which (is .nan (prop_12 [, "Freq"])!=TRUE) ,]

p_util12<-as.vector (prop_u12[which (prop_u12[,2]==Valor_S[1] \& prop_u12

$[, 3]==$ Valor_S [2] \& prop_u12[,4]==Valor_S $[3] \quad \&$ prop_u12[,5]==Valor_S [4] 
\& prop_u12 $[, 6]==$ Valor_S [5] \& prop_u12[,7]==Valor_S [6] \& prop_u12 $[, 8]==$ Valor_S $[7] \&$ prop_u12[,9] $==$ Valor_S $[8] \&$ prop_u12[,10]== Valor_S [9] \& prop_u12[,11]==Valor_S[10] \& prop_u12[,12]==-Valor_S[11] \& prop_u12[, $\overline{13}]==$ Valor_S $[12] \overline{)}, 14])$

1064 1065

1066

1067

1068

1069

1070

1071

1072

1073

1074

1075

1076

1077

1078

1079

1080

1081

1082

1083

1084

1085

1086

1087

1088

1089

1090

1091

1092

1093

1094

1095

1096

1097

1098

1099

1100

1101

1102

1103

ifelse (length $\left(p_{\_}\right.$util12)!=0, p_util $<-p_{\_}$util12, ifelse $\left(\right.$length $\left(p_{-}\right.$util11)!=0, $p_{-}$util $<-p_{-}$util11, ifelse $\left(\overline{l e n g t h}\left(p_{-}\right.\right.$util10 $) !=0, p_{-}$util $<-p_{-}$util10,

ife- $\overline{s e}\left(\operatorname{length}\left(p_{-}\right.\right.$util9 $) !=0, p_{-}$util $<-\bar{p}_{-}$util9, ifelse $\left(\operatorname{length}\left(p_{-}\right.\right.$util 8$) !=0$, $p_{-}$util $<-p_{-}$utils, ifelse $\left(\operatorname{length}\left(p_{-}\right.\right.$util 7$) !=0, p_{-}$util $<-p_{-}$util $\overline{\text {, }}$, ifelse ( length $\left(p_{-}\right.$util6 $) !=0, p_{-} u t i l<-p_{-} u t i l 6, \quad$ ifelse $\left(\operatorname{length}\left(p_{-}\right.\right.$util 5$) !=0, p_{-}$util $<-p_{-}$util5, ifelse (length $\left(p_{-}\right.$util4)! =0, p_util $<-p_{-}$util 4 , ifelse (length ( $p_{-}$util3 $) !=0, p_{-} u t i l<-p_{-} u t i l 3$, ifelse $\left(\right.$ length $\left(p_{-}\right.$util 2$) !=0, p_{-} u t i l<-$

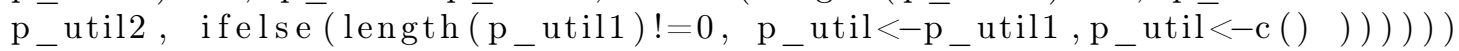
)$\overline{())) ~(~}$

ifelse $\left(\operatorname{length}\left(p_{-}\right.\right.$util $)==0, \operatorname{conta} 12<-\operatorname{conta} 12+1,\{$

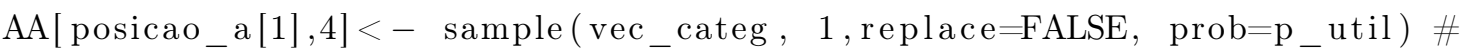
estimativa de novos valores

AA[posicao_a[1],21]<-p_util[1] \# a probabilidade não condicional utilizada para estimativa da categoria 1: Level

AA[posicao_a[1],22]<-p_util[2] \# a probabilidade não condicional utilizada para estimativa da categoria 2: Level

$\mathrm{AA}[$ posicao_a[1],23]<- p_util [3] \# a probabilidade não condicional utilizada para estimativa da categoria 3: Level

AA[posicao_a[1],24]<- p_util[1]*(1-p_util[1]) \# a incerteza relacionada a categoria 1: Level

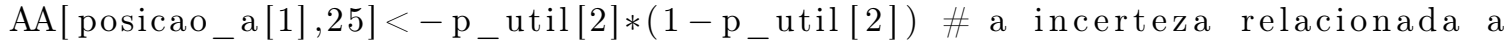
categoria 2: Level

$\mathrm{AA}_{[}$posicao_a[1],26]<- _ $_{-}$util $[3] *\left(1-\mathrm{p}_{-}\right.$util [3]) \# a incerteza relacionada a categoria 3: Level

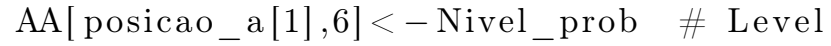

$\mathrm{AA}[$ posicao_a[1],7]<-Valor_S [1]

$\mathrm{AA}[$ posicao_a [1],8]<-Valor_S [2]

$\mathrm{AA}[$ posicao_a [1],9]<-Valor_S [3]

$\mathrm{AA}[$ posicao_a [1],10]<-Valor_S [4]

$\mathrm{AA}[$ posicao_a [1],11]<-Valor_S [5]

$\mathrm{AA}[$ posicao_a [1],12]<-Valor_S [6]

$\mathrm{AA}[$ posicao_a [1],13]<-Valor_S [7]

$\mathrm{AA}[$ posicao_a [1],14]<-Valor_S [8]

$\mathrm{AA}[$ posicao_a [1],15]<-Valor_S [9]

AA[posicao_a[1],16]<-Valor_S [1 0$]$

$\mathrm{AA}[$ posicao_a[1],17]<-Valor_S [11]

$\mathrm{AA}[$ posicao_a [1],18]<-Valor_S [1 2]

AA[posicao_a [1],31] $<-$ posto_S [1]

$\mathrm{AA}[$ posicao_a [1],32]<- posto_S [2]

$\mathrm{AA}[$ posicao_a [1],33]<- posto_S [3]

$\mathrm{AA}[$ posicao_a [1],34]<-posto_S [4]

$\mathrm{AA}[$ posicao_a [1],35]<-posto_S [5]

$\mathrm{AA}[$ posicao_a [1],36]<-posto_S [6]

$\mathrm{AA}[$ posicao_a [1],37]<- posto_S [7]

$\mathrm{AA}[$ posicao_a [1],38]<-posto_S [8]

AA[posicao_a [1],39]<-posto_S [9]

AA $[$ posicao_a $[1], 40]<-$ posto_S $[10]$

$\mathrm{AA}[$ posicao_a [1],41]<-posto_S [11]

AA[posicao_a[1],42]<-posto_S [12]

posicao_a $a<-$ posicao_a $[-1]$

\}) 
$1104\}$,

$1105\{\operatorname{conta} 0<-\operatorname{conta} 0+1$

$1(06(3))))$ )) )) )) )

1107

1108 \} ) \# fim do if que o Nivel de Probabilidade estava em 0

1109

1110 posicao_a $<-$ sample (posicao_a)

1111 conta sem $<-$ conta $s e m+1$

1112

1113

$1114\}$, conta $14<-$ conta $14+1)$

1115

1116 posicao_a $<-$ sample (posicao_a)

1117 conta_sem $<-$ conta_sem +1

1118

1119 \} \# final do while posicao_a[1]

1120

1121

1122 for(i in $1:$ length(vetor_amostras)) \{

1123

1124 ifelse (AA[vetor_amostras [i], 4$]==1,\{$

1125 AA[vetor_amostras [i],21]<-1 \# 100\% de acontecer a categoria 1

1126 AA[vetor_amostras [i],22]<-0 \# $0 \%$ de acontecer a categoria 2

1127 AA[vetor_amostras[i],23]<-0 \#0\% de acontecer a categoria 3

1128 AA[vetor_amostras [i] 24$]<-0 \quad$ \# categoria 1 sem incerteza

1129 AA[vetor_amostras[i],25]<-0 \# categoria 2 sem incerteza

$1130 \mathrm{AA}[$ vetor amostras [i] 26$]<-0 \quad$ \# categoria 3 sem incerteza

1131 AA[vetor_amostras [i],6]<-0 \# Level 0

1132

$1133\}$, ifelse $(\mathrm{AA}[$ vetor amostras $[\mathrm{i}], 4]==2,\{$

1134 AA[vetor_amostras $[\mathrm{i}], 21]<-0 \quad \# 0 \%$ de acontecer a categoria 1

1135 AA[vetor_amostras[i],22]<-1 \# 100\% de acontecer a categoria 2

1136 AA[vetor_amostras[i],23]<-0 \# 0\% de acontecer a categoria 3

1137 AA[vetor_amostras[i],24]<-0 \# categoria 1 sem incerteza

1138 AA[vetor_amostras[i],25]<-0 \# categoria 2 sem incerteza

1139 AA[vetor_amostras [i] 26$]<-0 \quad$ \# categoria 3 sem incerteza

1140 AA[vetor_amostras [i],6]<-0 \# Level 0

$1141\},\{$

1142 AA[vetor_amostras [i],21] $<-0$

\# $0 \%$ de acontecer a categoria 1

1143 AA[vetor_amostras [i] 22$]<-0$

1144 AA[vetor_amostras [i] 23$]<-1$

1145 AA[vetor_amostras [i], 24] $<-0$

1146 AA[vetor_amostras [i], 25] $<-0$

1147 AA[vetor_amostras [i], 26] $<-0$

1148 AA[vetor_amostras [i] 6$]<-0$

\# $0 \%$ de acontecer a categoria 2

\# $0 \%$ de acontecer a categoria 3

\# categoria 1 sem incerteza

\# categoria 2 sem incerteza

\# categoria 3 sem incerteza

$1149 \quad\}))$

$1150\}$

1151

1152

1153 \# recategorizando a incerteza em 0 e 1 quando for $>=0.15$

1154

1155 for ( $\mathrm{i}$ in $1:$ nrow $(\mathrm{AA}))\{$

1156 if else $(\mathrm{AA}[\mathrm{i}, 24]>=0.20, \mathrm{AA}[\mathrm{i}, 27]<-4, \mathrm{AA}[\mathrm{i}, 27]<-\mathrm{AA}[\mathrm{i}, 4])$

1157 if els e $(\mathrm{AA}[\mathrm{i}, 25]>=0.20, \mathrm{AA}[\mathrm{i}, 28]<-4, \mathrm{AA}[\mathrm{i}, 28]<-\mathrm{AA}[\mathrm{i}, 4])$

1158 if else $(\mathrm{AA}[\mathrm{i}, 26]>=0.20, \mathrm{AA}[\mathrm{i}, 29]<-4, \mathrm{AA}[\mathrm{i}, 29]<-\mathrm{AA}[\mathrm{i}, 4])$

1159 \}

1160 for ( $\mathrm{i}$ in $1: \operatorname{nrow}(\mathrm{AA}))$ \{

1161 ifelse $(\mathrm{AA}[\mathrm{i}, 24]>=0.20 \quad \mid \mathrm{AA}[\mathrm{i}, 25]>=0.20 \quad \mathrm{AA}[\mathrm{i}, 26]>=0.20, \mathrm{AA}[\mathrm{i}, 20]<-4, \mathrm{AA}$ $[\mathrm{i}, 20]<-\mathrm{AA}[\mathrm{i}, 4])$ 
$1162\}$

1163

1164

1165 exportar $<-$ data. frame (AA)

1166

1167 names(exportar) [1] $<-$ "i_x"

1168 names(exportar) $[2]<-" j$ j_y"

1169 names(exportar) $[3]<-$ "k_z"

1170 names(exportar) $[4]<-$ "categ"

1171 names(exportar) [5]<- "pos_inic"

1172 names(exportar) [6]<- "Level"

1173 names(exportar) [7]<- "S(u1)"

1174 names(exportar) [8]<- "S(u2)"

1175 names(exportar) $[9]<-$ "S(u3)"

1176 names(exportar) $[10]<-$ "S(u4)"

1177 names(exportar) [11]<- "S(u5)"

1178 names(exportar) [12]<- "S(u6)"

1179 names (exportar) [13]<- "S(u7)"

1180 names (exportar) $[14]<-$ "S (u8)"

1181 names(exportar) $[15]<-$ "S(u9)"

1182 names(exportar) $[16]<-$ "S(u10)"

1183 names(exportar) [17]<- "S(u11)"

1184 names(exportar) [18]<- "S(u12)"

1185

1186 names(exportar) $[20]<-$ "Incertezas"

1187 names(exportar) $[21]<-$ "Prob_categ_1"

1188 names(exportar) [22]<- "Prob_categ_2"

1189 names(exportar) [23]<- "Prob_categ_3"

1190 names(exportar) $[24]<-$ "Incerteza_categ_1"

1191 names(exportar) $[25]<-$ "Incerteza_categ_2"

1192 names (exportar) $[26]<-$ "Incerteza_categ_3"

1193 names(exportar) $[27]<-$ "Incert_0 $\overline{1}_{-}$categ 1 "

1194 names(exportar) $[28]<-$ "Incert_o ${ }_{-}{ }_{1}$ categ_ 2 "

1195 names (exportar) $[29]<-$ "Incert_ $0{ }_{-}{ }_{-}{ }_{-}$categ_ $3 "$

1196 names (exportar) $[31]<-$ "Posto ${ }^{1} "$

1197 names(exportar) $[32]<-$ "Posto_2"

1198 names(exportar) $[33]<-$ "Posto_3"

1199 names (exportar) $[34]<-$ "Posto_4"

1200 names(exportar) $[35]<-$ "Posto_5"

1201 names (exportar) $[36]<-$ "Posto_6"

1202 names(exportar) $[37]<-$ "Posto_7"

1203 names(exportar) $[38]<-$ "Posto_8"

1204 names(exportar) $[39]<-$ "Posto_9"

1205 names(exportar) $[40]<-$ "Posto_ $10 "$

1206 names (exportar) [41]<- "Posto_ $11 "$

1207 names (exportar) $[42]<-$ "Posto_ $12 "$

1208

1209

1210 \# Salvar os resultados assim como:

1211

1212 \#write . table (exportar, "C: $\backslash \backslash$ Users $\backslash \backslash$ joan $\backslash \backslash$ Documents $\backslash \backslash \operatorname{Programas} \backslash \backslash$

Dado_sintético_2D $\backslash \backslash$ figuras $\backslash \backslash$ Colocar_na_tese $\backslash \backslash$ realizacoes $\backslash \backslash$ realizacao 2.

1213

1214 \#save.image ("C: $\backslash \backslash$ Users $\backslash \backslash$ joan $\backslash \backslash$ Documents $\backslash \backslash$ Programas $\backslash \backslash$ Dado_sintético_2D $\backslash \backslash$

figuras $\backslash \backslash$ Colocar_na_tese $\backslash \backslash$ realizacao $2 ")$

1215

1216

1217 \# estudo sobre a saida dos resultados 
1218

1219 hist (exportar [, "Level"]) \# histograma das probabilidades condicionais utilizadas na estimativa das celulas

1220

1221 prob_condi<-data.frame(prop.table (table (exportar [, "Level"])) ) \# As probabilidades condicionais utilizadas na estimativa das celulas

1222

1223 prob_condi

1224

1225 \# resultados

1226 head (exportar)

1227 open $3 \mathrm{~d}()$

1228 plot3d (exportar [,1], exportar [,2], exportar [,3], col=exportar [,4], type='s , radius $=\mathrm{c}(0.55,0.55,0.55), \mathrm{xlab}=" \mathrm{X}^{\prime}, \quad$ ylab="Y", zlab="Z" ) \#resultados

1229

1230

1231 \# Imagem de treinamento

1232 open3d() \# função para preparar o ambiente de gráficos

1233 plot3d (BB $[, 1], \mathrm{BB}[, 2], \mathrm{BB}[, 3], \operatorname{col}=\mathrm{BB}[, 4]$, type='s', radius=c

$(0.55,0.55,0.55), x l a b=" X ", \quad y l a b=" Y ", \quad z l a b=" Z ")$ \# função que gráfica em $3 \mathrm{D}$

1234

1235 \# população

1236 open3d() \# função para preparar o ambiente de gráficos

1237 plot3d (bb $[, 1]$, bb $[, 2]$, bb $[, 3], \operatorname{col}=b b[, 4]$, type='s', radius=c

$\left.(0.55,0.55,0.55), x l a b=" X^{\prime \prime}, y l a b=" Y ", \quad z l a b=" Z "\right)$ \# função que gráfica em $3 \mathrm{D}$

1238

1239 \# amostras

1240 open3d() \# função para preparar o ambiente de gráficos

1241 plot3d(bb1 [,1], bb1 [,2], bb1 [,3], col=bb1[,4], type='s', radius=c

$(0.55,0.55,0.55), x l a b=" X ", \quad y l a b=" Y ", \quad z l a b=" Z ")$ \# função que gráfica em $3 \mathrm{D}$

1242

1243 \# Incertezas intercetadas

1244 open3d() \# função para preparar o ambiente de gráficos

1245 plot3d (exportar [,1], exportar [,2], exportar [,3], col=exportar [,20], type=' $\mathrm{s}^{\prime}$, radius $\left.=\mathrm{c}(0.55,0.55,0.55), \mathrm{xlab}=" \mathrm{X}^{\prime}, \quad \mathrm{lab}=" \mathrm{Y}^{\prime}, \mathrm{zlab}=" \mathrm{Z} "\right)$ \#resultados

1246

1247 \# Incerteza associada a 1

1248 open3d() \# função para preparar o ambiente de gráficos

1249 plot3d (exportar [,1], exportar [,2], exportar [,3], col=exportar [,27], type=' $\mathrm{s}^{\prime}, \operatorname{radius}=\mathrm{c}(0.55,0.55,0.55), \mathrm{xlab}=" \mathrm{X}$, $\left., \mathrm{ylab}=" \mathrm{Y}^{\prime}, \mathrm{zlab}=" \mathrm{Z} "\right)$ \#resultados

1250

1251 \# Incerteza associada a 2

1252 open3d() \# função para preparar o ambiente de gráficos

1253 plot3d (exportar [,1], exportar [,2], exportar [,3], col=exportar [,28], type=' $\mathrm{s}^{\prime}$, radius $=\mathrm{c}(0.55,0.55,0.55), \mathrm{xlab}=" \mathrm{X}$, $\left., \quad \mathrm{lab}=" \mathrm{Y}^{\prime}, \mathrm{zlab}=" \mathrm{Z} "\right)$ \#resultados

1254

1255 \# Incerteza associada a 3

1256 open3d() \# função para preparar o ambiente de gráficos

1257 plot3d (exportar [,1], exportar [,2], exportar [,3], col=exportar [,29], type='

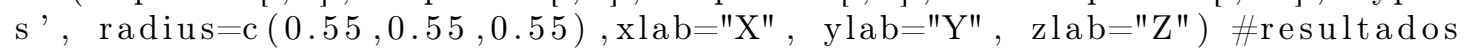

1258

1259 \# Salve a imagem do programa para visualização posterior

1260

1261 \#save .image ("C: $\backslash \backslash$ Users $\backslash \backslash$ joan $\backslash \backslash$ Documents $\backslash \backslash$ Programas $\backslash \backslash$ Dado_sintético_2D $\backslash$ _ figuras $\backslash \backslash$ Colocar_na_tese $\backslash \backslash$ overview") 